\title{
New Biphenol-based Phosphoramidite Ligands for the Enantioselective Copper-Catalyzed Conjugate Addition of Diethylzinc
}

\author{
Alexandre Alexakis,* Damien Polet, Stéphane Rosset, Sébastien March \\ Department of Organic Chemistry, University of Geneva, 30 quai Ernest Ansermet, Genève 4, \\ Switzerland $\mathrm{CH}-1211$
}

FAX : (41 22) 3287396 ; e-mail : alexandre.alexakis@chiorg.unige.ch

\section{Contents of Supporting Information}

(58 pages)

Page S1 : Title of the paper, author's name and address along with the contents

Page S2 : General procedures, ligand synthesis procedure and asymmetric conjugate addition procedure

Pages S3-4 : ${ }^{1} \mathrm{H}$ and ${ }^{13} \mathrm{C}$ NMR spectra of compound $\mathbf{B} 2$

Pages S5-6 : ${ }^{1} \mathrm{H}$ and ${ }^{13} \mathrm{C}$ NMR spectra of compound $\mathbf{B} 3$

Pages S7-8: ${ }^{1} \mathrm{H}$ and ${ }^{13} \mathrm{C}$ NMR spectra of compound $\mathbf{B} 4$

Pages S9-10: ${ }^{1} \mathrm{H}$ and ${ }^{13} \mathrm{C}$ NMR spectra of compound $\mathbf{1}$

Pages S11-12: ${ }^{1} \mathrm{H}$ and ${ }^{13} \mathrm{C}$ NMR spectra of compound 2

Pages S13-14: ${ }^{1} \mathrm{H}$ and ${ }^{13} \mathrm{C}$ NMR spectra of compound $\mathbf{B 5}$

Pages S15-16: ${ }^{1} \mathrm{H}$ and ${ }^{13} \mathrm{C}$ NMR spectra of compound $\mathbf{B 6}$

Pages S17-18: ${ }^{1} \mathrm{H}$ and ${ }^{13} \mathrm{C}$ NMR spectra of compound $\mathbf{B} 7$

Pages S19-20: ${ }^{1} \mathrm{H}$ and ${ }^{13} \mathrm{C}$ NMR spectra of compound 3

Pages S21-22: ${ }^{1} \mathrm{H}$ and ${ }^{13} \mathrm{C}$ NMR spectra of compound $\mathbf{B 8}$

Pages S23-24: ${ }^{1} \mathrm{H}$ and ${ }^{13} \mathrm{C}$ NMR spectra of compound 4

Pages S25-26: ${ }^{1} \mathrm{H}$ and ${ }^{13} \mathrm{C}$ NMR spectra of compound $\mathbf{B 9}$

Pages S27-28: ${ }^{1} \mathrm{H}$ and ${ }^{13} \mathrm{C}$ NMR spectra of compound 5

Pages S29-30: ${ }^{1} \mathrm{H}$ and ${ }^{13} \mathrm{C}$ NMR spectra of compound B10

Pages S31-32 : ${ }^{1} \mathrm{H}$ and ${ }^{13} \mathrm{C}$ NMR spectra of compound $\mathbf{B 1 1}$

Pages S33-35 : ${ }^{1} \mathrm{H},{ }^{13} \mathrm{C}$ and ${ }^{31} \mathrm{P}$ NMR spectra of compound L3a

Pages S36-38: ${ }^{1} \mathrm{H},{ }^{13} \mathrm{C}$ and ${ }^{31} \mathrm{P}$ NMR spectra of compound L4a

Pages S39-41: ${ }^{1} \mathrm{H},{ }^{13} \mathrm{C}$ and ${ }^{31} \mathrm{P}$ NMR spectra of compound L5a

Pages S42-44: ${ }^{1} \mathrm{H},{ }^{13} \mathrm{C}$ and ${ }^{31} \mathrm{P}$ NMR spectra of compound $\mathbf{L 6 a}$

Pages S45-47: ${ }^{1} \mathrm{H},{ }^{13} \mathrm{C}$ and ${ }^{31} \mathrm{P}$ NMR spectra of compound $\mathbf{L 7 a}$

Pages S48-50: ${ }^{1} \mathrm{H},{ }^{13} \mathrm{C}$ and ${ }^{31} \mathrm{P}$ NMR spectra of compound L8a

Pages S51-53: ${ }^{1} \mathrm{H},{ }^{13} \mathrm{C}$ and ${ }^{31} \mathrm{P}$ NMR spectra of compound $\mathbf{L 9 a}$

Pages S54-56: ${ }^{1} \mathrm{H},{ }^{13} \mathrm{C}$ and ${ }^{31} \mathrm{P}$ NMR spectra of compound $\mathbf{L 9 b}$

Pages S57-59: ${ }^{1} \mathrm{H},{ }^{13} \mathrm{C}$ and ${ }^{31} \mathrm{P}$ NMR spectra of compound L10a

Pages S60-62: : ${ }^{1} \mathrm{H},{ }^{13} \mathrm{C}$ and ${ }^{31} \mathrm{P}$ NMR spectra of compound L10b

Pages S63-65: ${ }^{1} \mathrm{H},{ }^{13} \mathrm{C}$ and ${ }^{31} \mathrm{P}$ NMR spectra of compound L11a 
General Procedures. ${ }^{1} \mathrm{H}$ (300, 400, and $\left.500 \mathrm{MHz}\right),{ }^{13} \mathrm{C}(75,100$, and $125 \mathrm{MHz})$, and ${ }^{31} \mathrm{P}$ NMR (122, 162, and $202 \mathrm{MHz}$ ) spectra were recorded in $\mathrm{CDCl}_{3}$ or DMSO-d6 and chemical shifts $(\delta)$ are quoted in ppm relative to tetramethylsilane $(0 \mathrm{ppm})$ and referenced to the residual undeuterated solvent. For convenience, the following abbreviations are used; s singlet, $\mathrm{d}$ - doublet, $\mathrm{t}$ - triplet, $\mathrm{q}$ - quartet, qn - quintet, $\mathrm{m}$ - multiplet, dd - doublet of doublet, $\mathrm{dt}$ - doublet of triplet, $\mathrm{td}$ - triplet of doublet, ddd - doublet of doublet of doublet. Coupling constants $(J)$ are given in Hertz $(\mathrm{Hz})$.

Optical rotations were measured at $22^{\circ} \mathrm{C}$ in a $10 \mathrm{~cm}$ cell in the stated solvent; $[\alpha]_{\mathrm{D}}$ values are given in $10^{-1} \mathrm{deg} \mathrm{cm}^{2} \mathrm{~g}^{-1}$ (concentration c given as $\mathrm{g} / 100 \mathrm{~mL}$ ). Enantiomeric excesses were determined by chiral GC (capillary column, $10 \mathrm{psi} \mathrm{H}_{2}$ ) or chiral SFC with the stated column. Temperature programs are described as follows: initial temperature $\left({ }^{\circ} \mathrm{C}\right)$ - initial time $(\mathrm{min})-$ temperature gradient $\left({ }^{\circ} \mathrm{C} / \mathrm{min}\right)$ - final temperature $\left({ }^{\circ} \mathrm{C}\right)$; retention time $\left(\mathrm{R}_{\mathrm{T}}\right)$ are given in min. Flash chromatography were performed using silica gel 32-63 $\mu \mathrm{m}, 60 \AA$.

Phosphorus trichloride was distilled once and then degassed three times in vacuo at $-78^{\circ} \mathrm{C}$ to remove the residual $\mathrm{HCl}$. It was stored at $-20^{\circ} \mathrm{C}$ in a Schlenk tube. Triethylamine was in every case freshly distilled. All non-solid phosphorus ligand were dissolved in the appropriate volume of dry toluene to make an about $1 \mathrm{M}$ solution that was used in conjugate addition reactions.

THF and diethyl ether were distilled from sodium using benzophenone ketyl as indicator. Other solvents were purified by standard techniques. Brine refers to a saturated solution of sodium chloride in water and DCM to dichloromethane.

Cyclohexenone S1, cycloheptenone S3, chalcone S4, benzalacetone S5, trans-5-Me-3-hexen2-one S6, trans-3-none-2-one S7, trans- $\beta$-nitrostyrene S8, trans-4-methyl- $\beta$-nitrostyrene S9, trans-4-methoxy- $\beta$-nitrostyrene S9, 1-(2-furyl)-2-nitroethylene S12, 1-nitrocyclohexene S13 2,4-dimethylphenol, 2,2'-dihydroxybiphenyl B1, 2,4-di-tert-butylphenol, 2-methoxy-4methylphenol and sec-butylamine were commercial products and used as received. Cyclopentadecen-2-one $\mathbf{S 3}$ was prepared according to the litterature. ${ }^{1}$

Melting points are uncorrected.

Ligand synthesis procedure. To a stirred mixture of $\mathrm{Et}_{3} \mathrm{~N}(111.1 \mathrm{mmol}, 15.5 \mathrm{~mL})$ and $\mathrm{PCl}_{3}$ (22.2 mmol, $1.9 \mathrm{~mL})$ at $0^{\circ} \mathrm{C}$, a solution of amine $(22.2 \mathrm{mmol})$ in THF $(10 \mathrm{~mL})$ was added and the reaction mixture was stirred for $3 \mathrm{~h}$ at room temperature. Biphenol $(22.2 \mathrm{mmol})$ in a solution of THF $(5 \mathrm{~mL})$ was slowly added to the reaction mixture at $0^{\circ} \mathrm{C}$ and then the suspension was stirred at RT overnight. The suspension was diluted in toluene $(8 \mathrm{~mL})$ and filtered on neutral alumina, the solution was concentrated and purified by flash chromatography through neutral alumina using dry toluene as eluent, to give the pure ligand as a white solid or a colorless oil.

Asymmetric conjugate addition procedure. To a solution of copper thiophenecarboxylate $(\mathrm{CuTC})^{2}(0.008 \mathrm{mmol})$ in $\mathrm{Et}_{2} \mathrm{O}(1 \mathrm{ml})$ at room temperature under nitrogen, was added the ligand $(0.0166 \mathrm{mmol})$ and $1 \mathrm{ml}$ of $\mathrm{Et}_{2} \mathrm{O}$. The solution was stirred at $25^{\circ} \mathrm{C}$ for $30 \mathrm{~min}$ and then cooled to $-30^{\circ} \mathrm{C}$. $\mathrm{Et}_{2} \mathrm{Zn}(1 \mathrm{ml}, 0.5 \mathrm{mmol} 15 \%$ in hexane) was added dropwise in order that temperature did not rise over $-30^{\circ} \mathrm{C}$. The solution was stirred for $5 \mathrm{~min}$, and the Michael acceptor $(0.415 \mathrm{mmol})$ was then added dropwise, neat or in solution in $0.5 \mathrm{~mL}$ of toluene. The reaction mixture was stirred at $-30^{\circ} \mathrm{C}$ for $12 \mathrm{~h}$ before being quenched by $2 \mathrm{~N}, \mathrm{HCl} / \mathrm{Et}_{2} \mathrm{O}$ (aq. sat. $\mathrm{NH}_{4} \mathrm{Cl}$ in the case of nitroalkene compounds). Enantiomeric excess was determined by chiral GC or SFC.

\footnotetext{
${ }^{1}$ Engman, L. J. Org. Chem. 1988, 53, 4031-4037.

${ }^{2}$ CuTC can either be purchased from Frontier Scientific Ltd or prepared according to the litterature procedure : Allred, G. D.; Liebeskind, L. S. J. Am. Chem. Soc. 1996, 118, 2748-2749.
} 


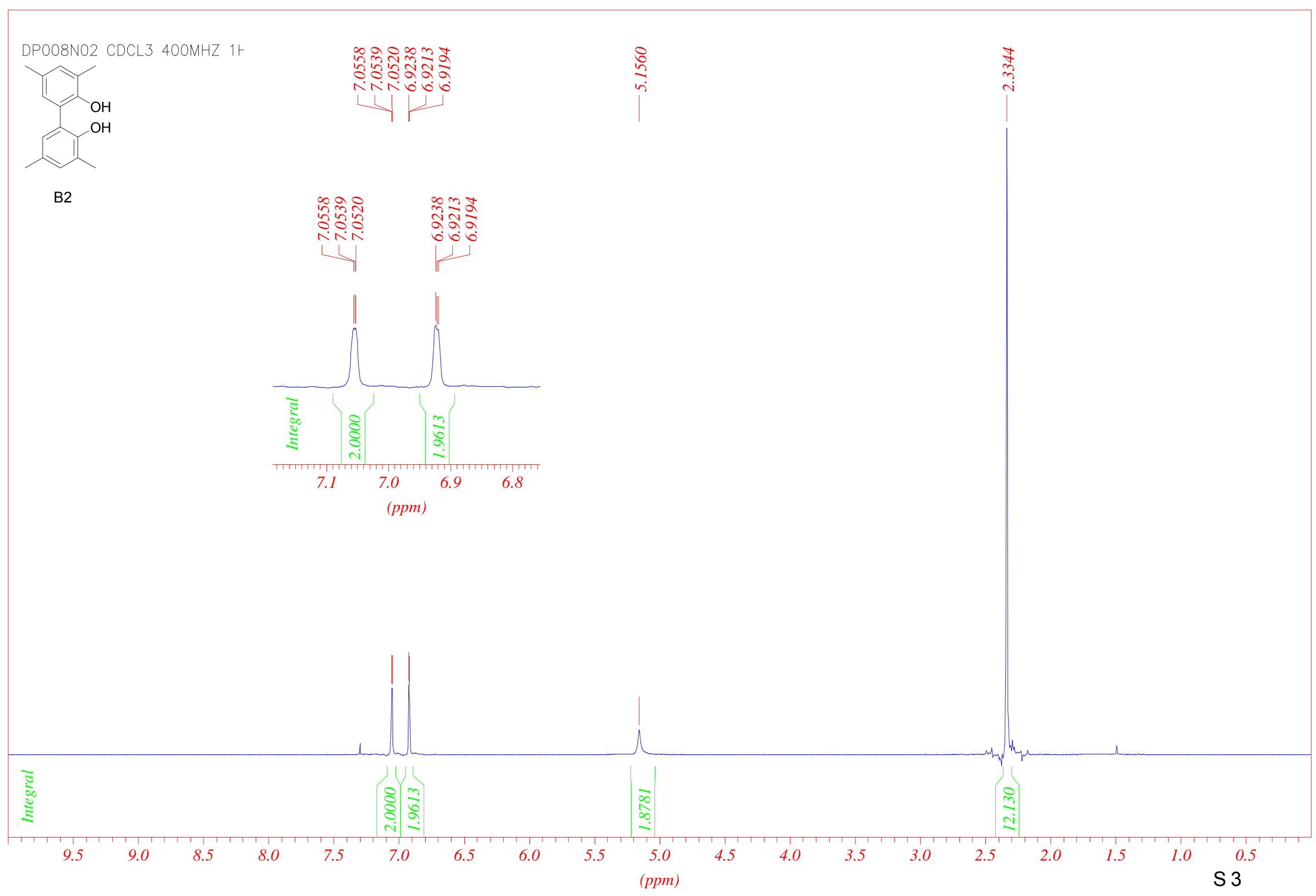




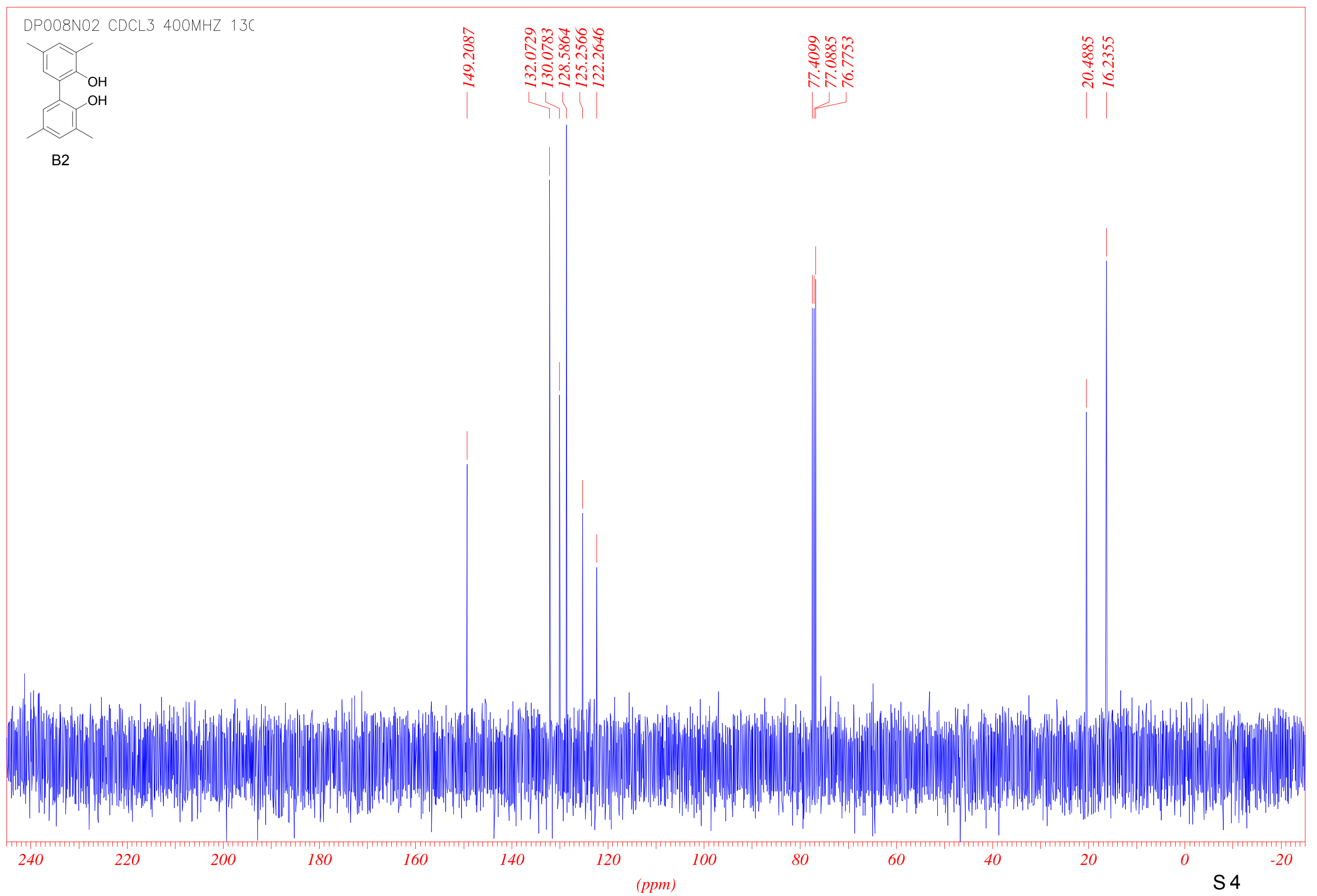




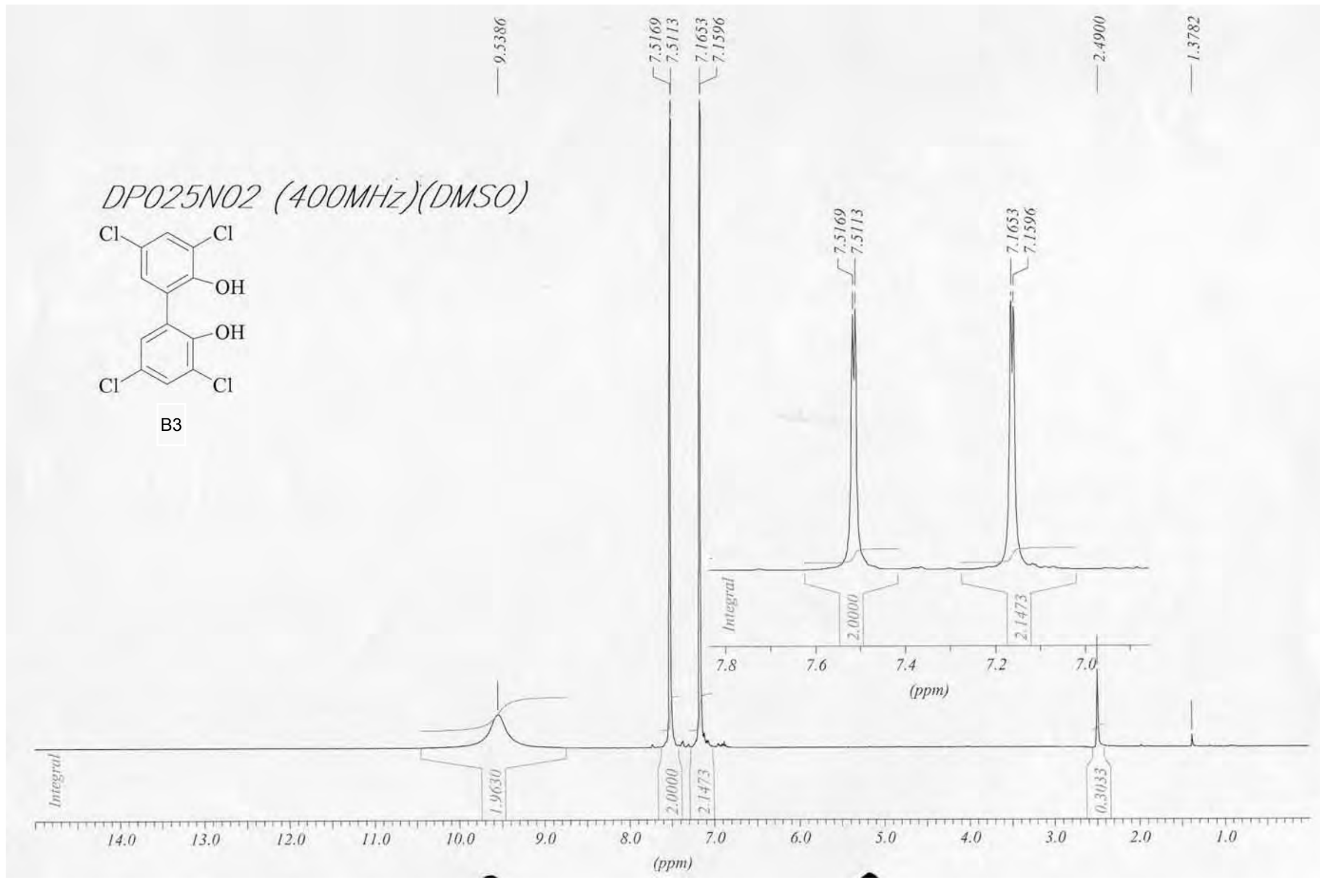




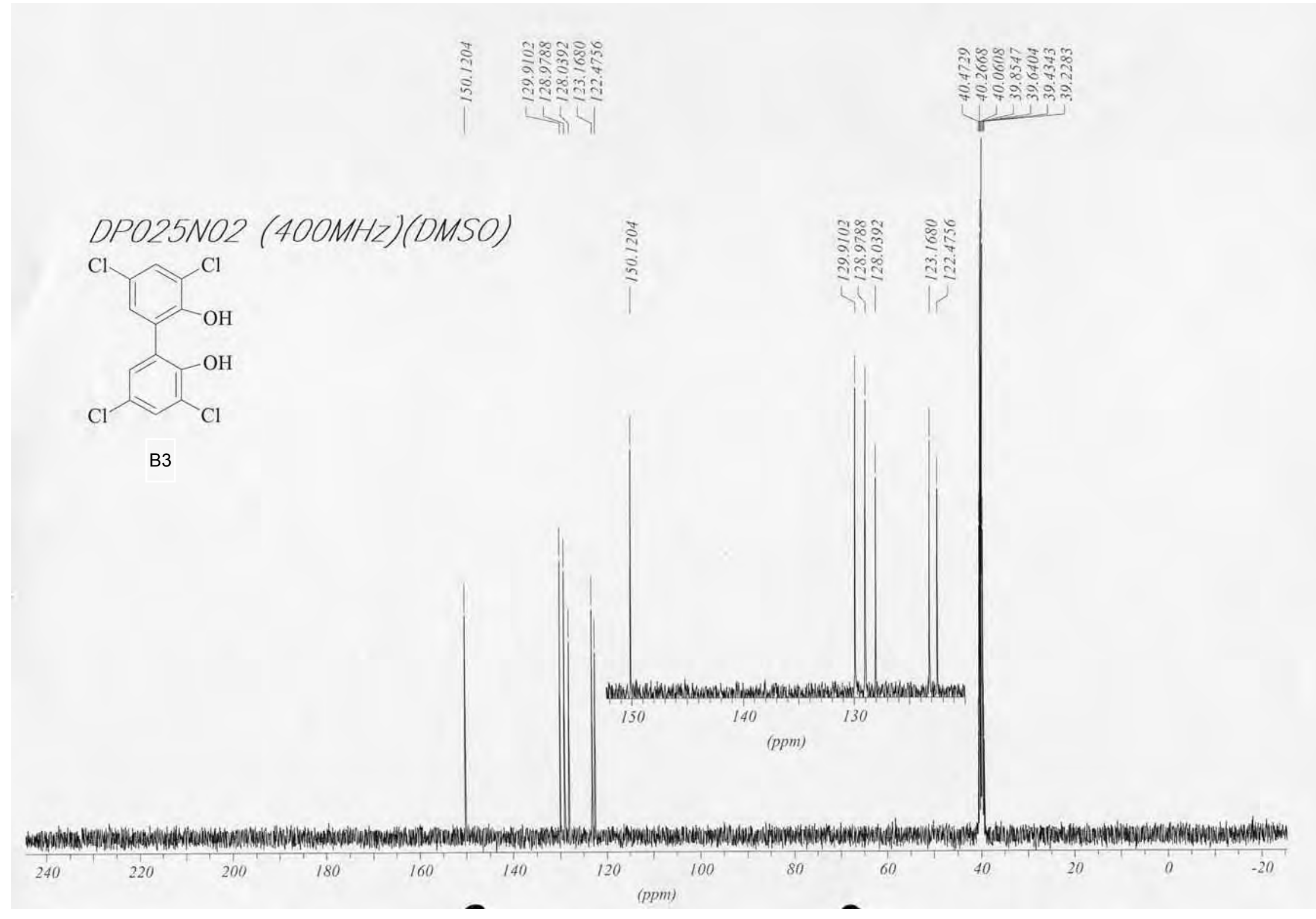


MARCH PRURON DASO uguest 3

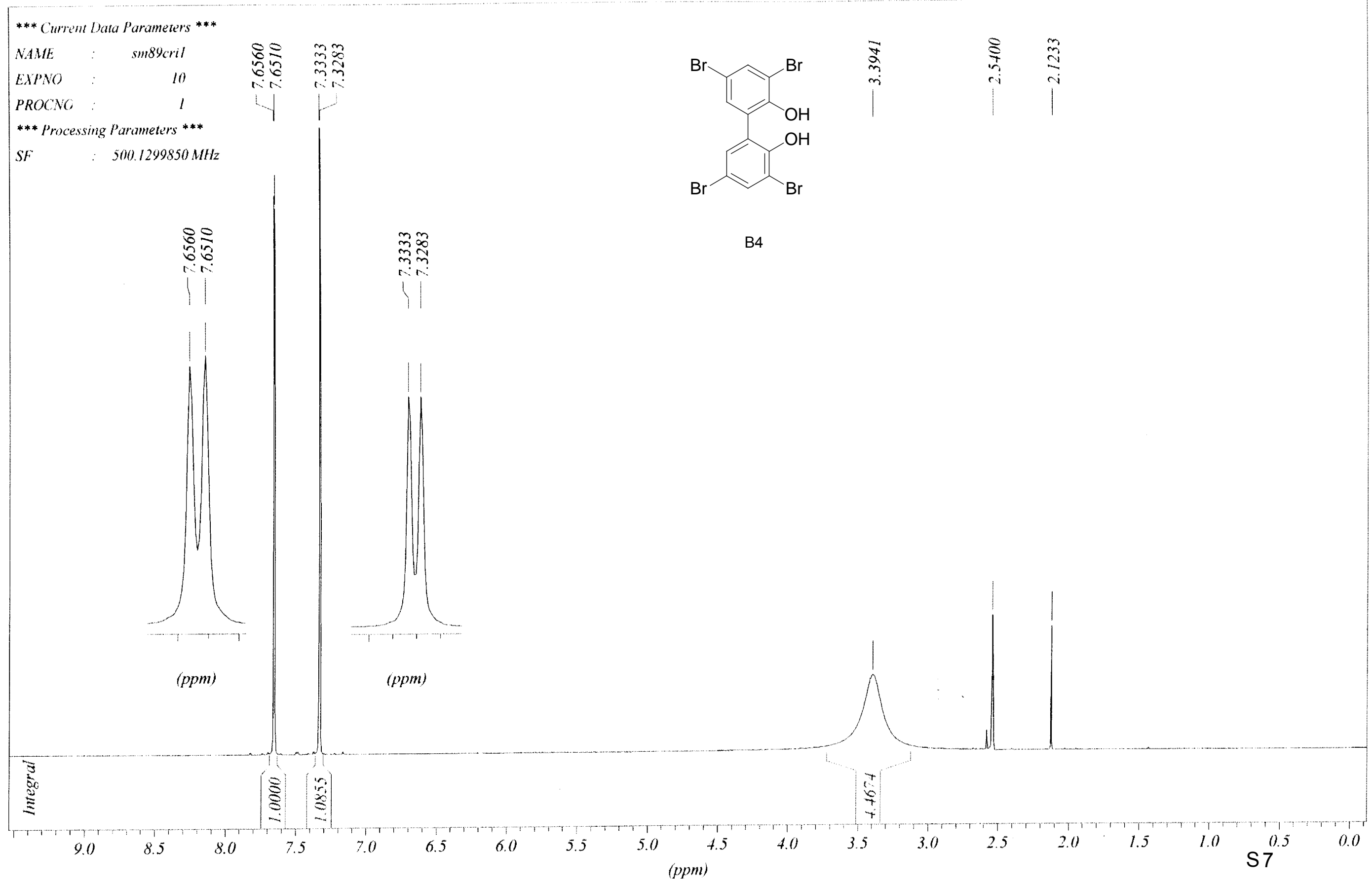




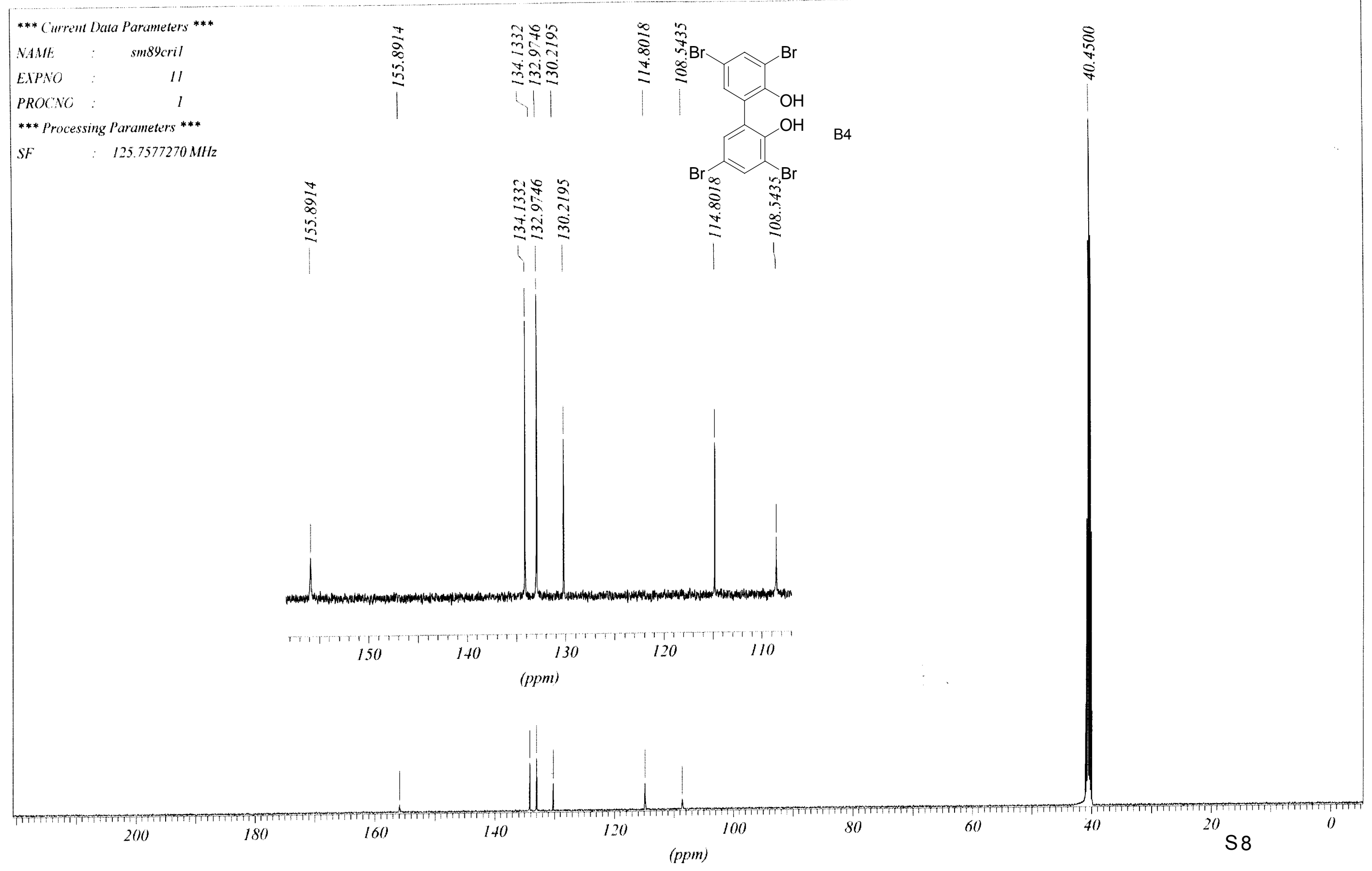




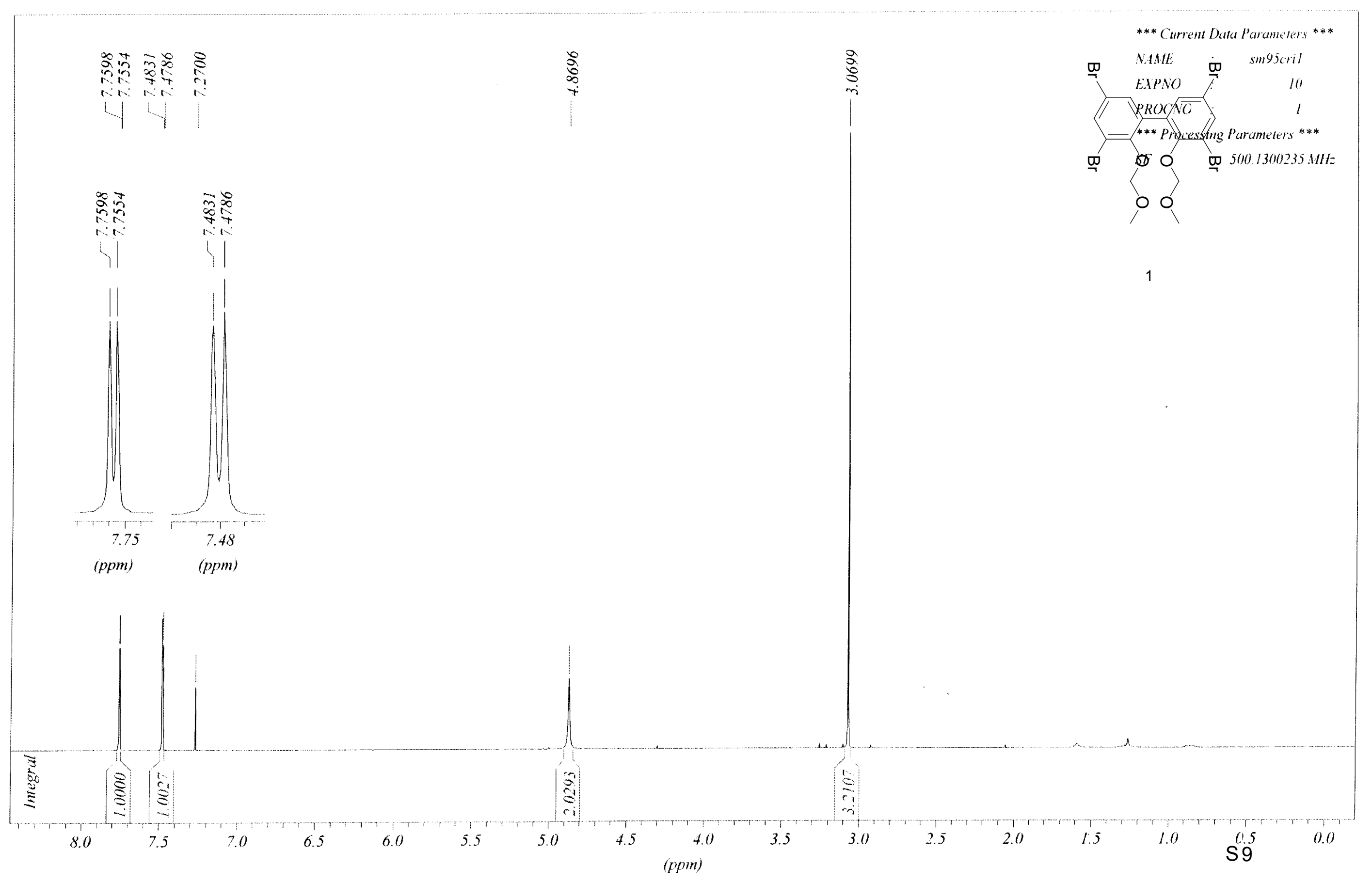




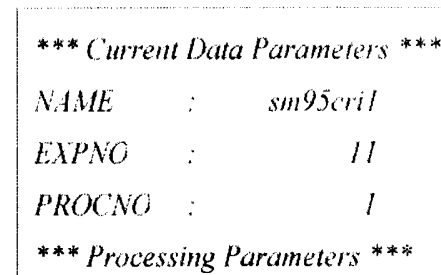

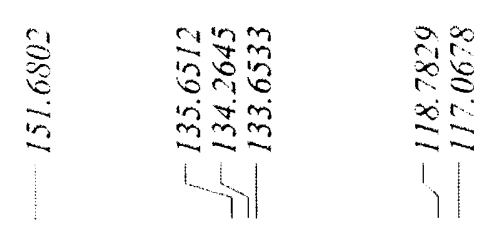

SF $\quad: 125.75779410 \mathrm{M} / 1 \mathrm{Iz}$

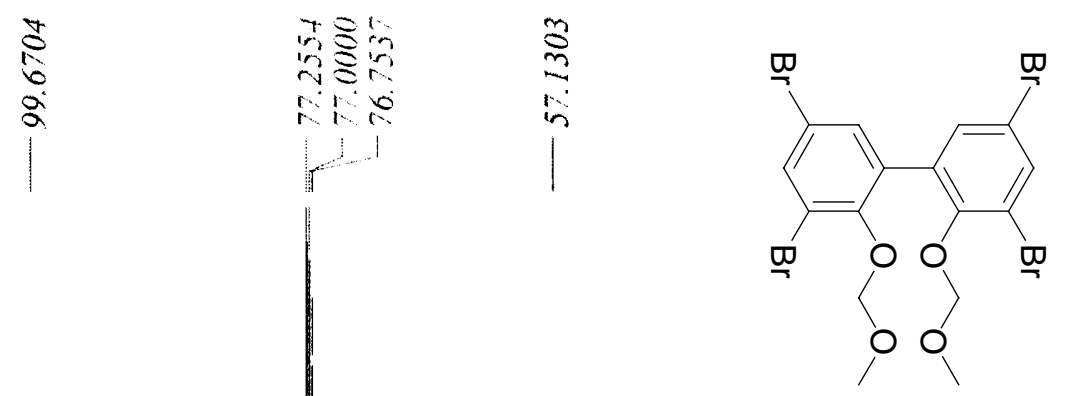

1
TrT,

200 


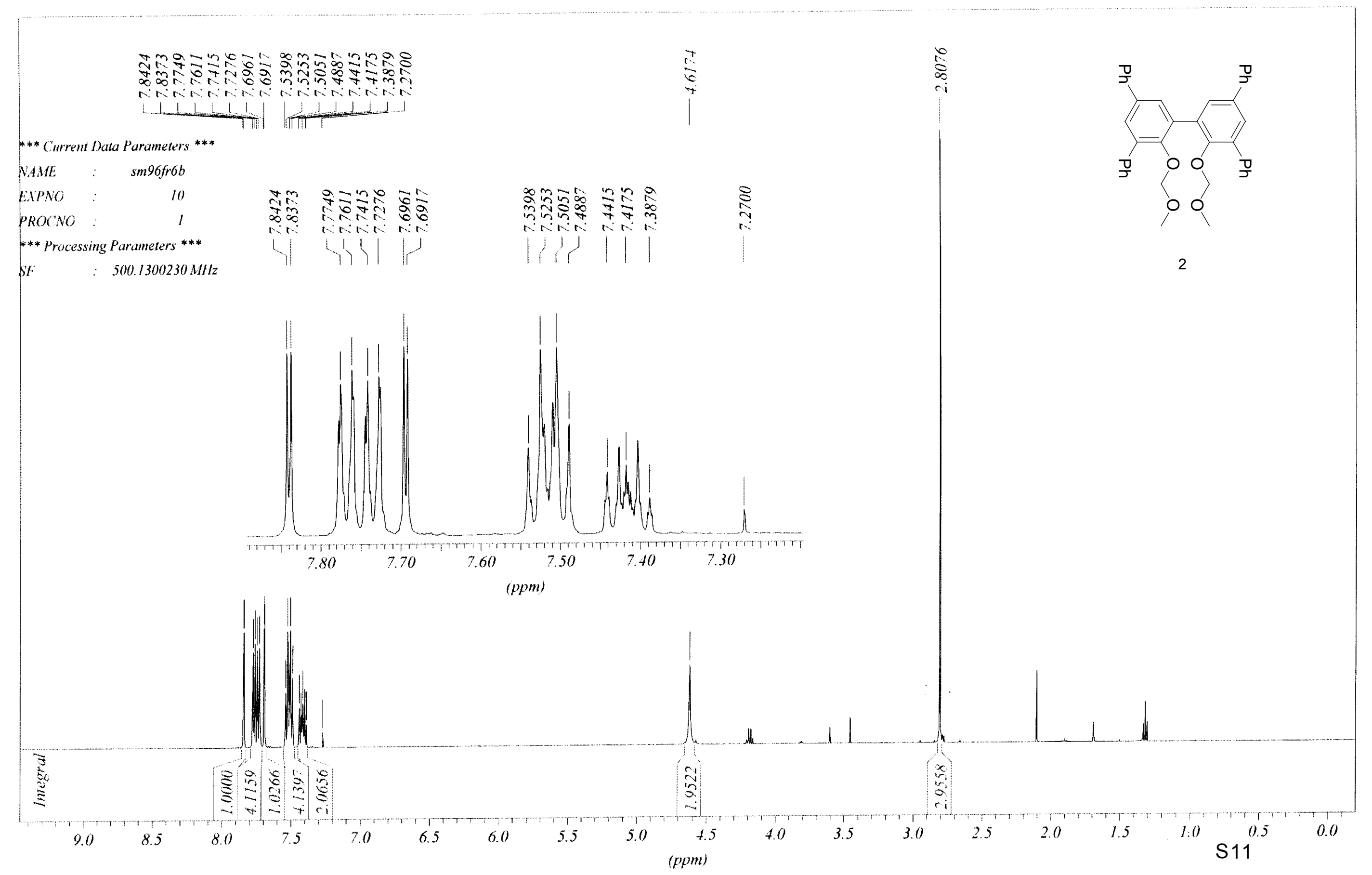




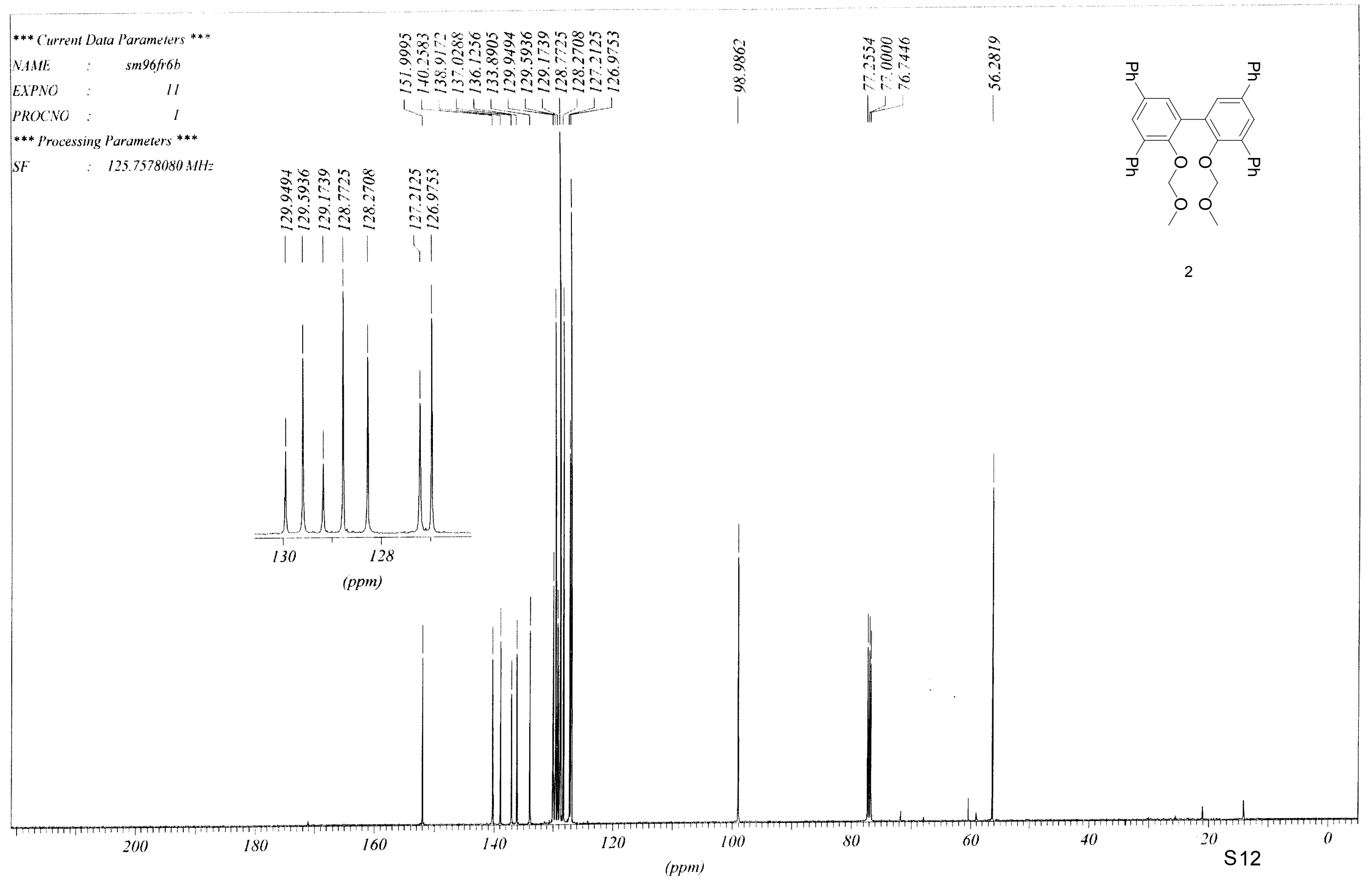




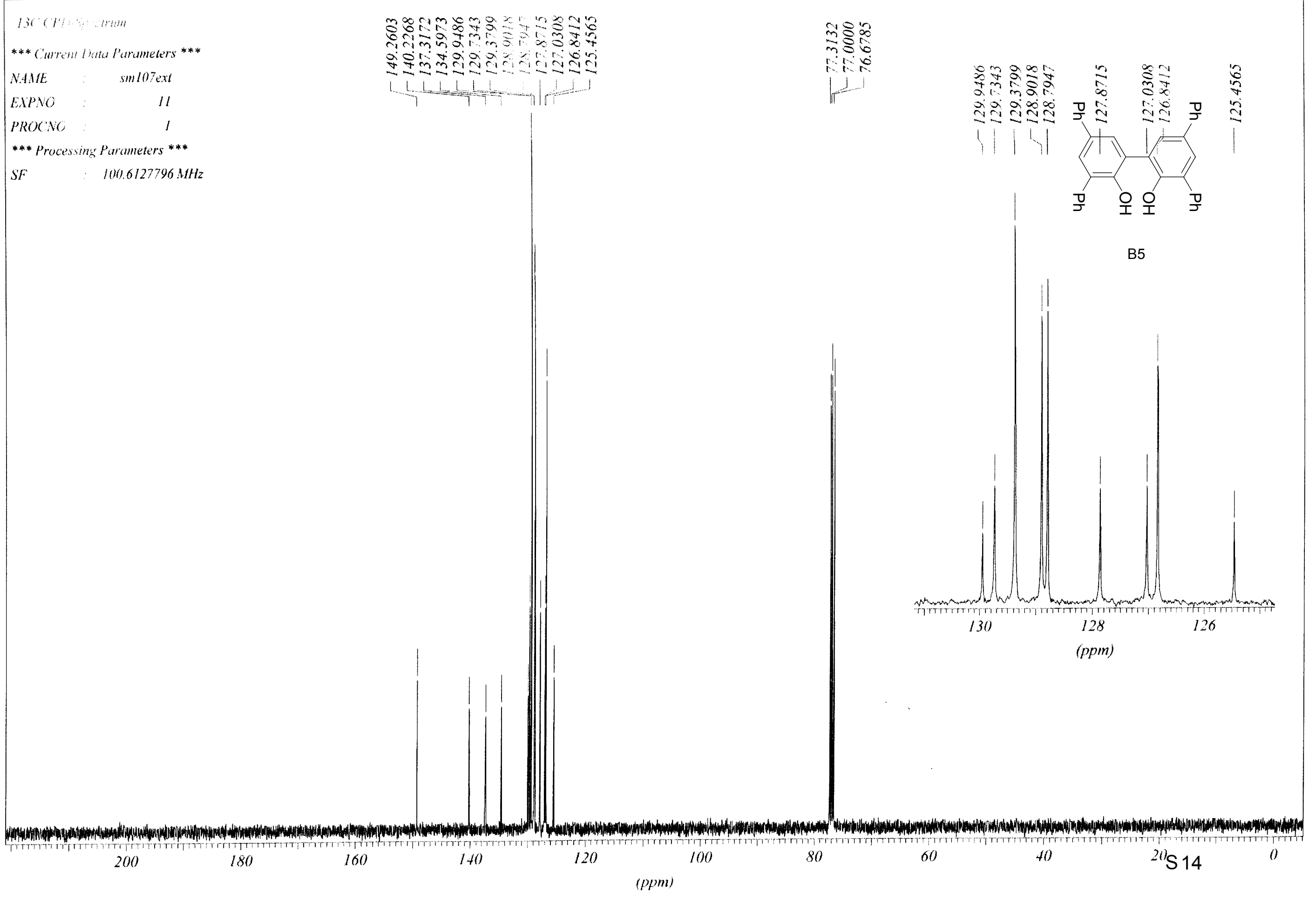




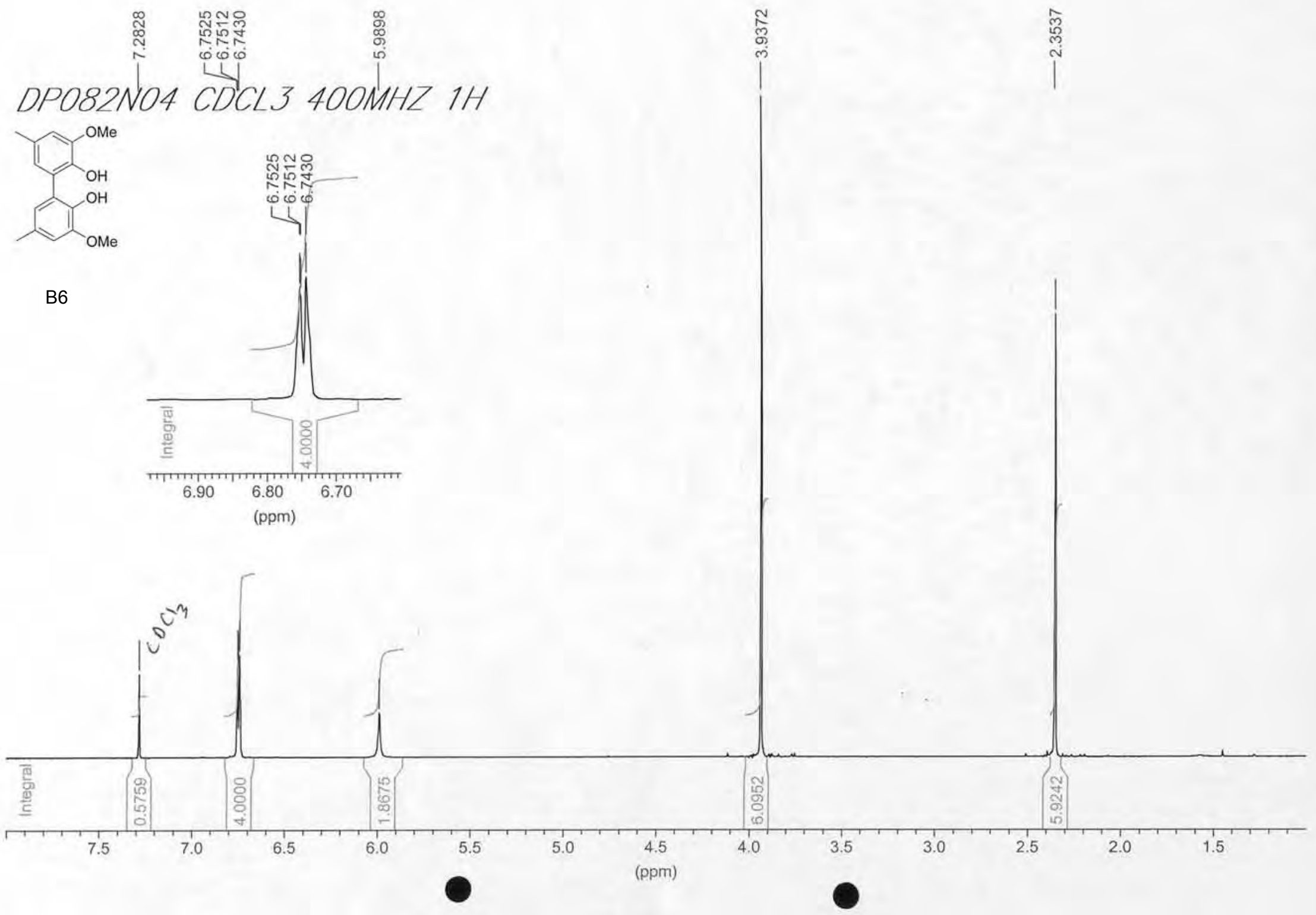




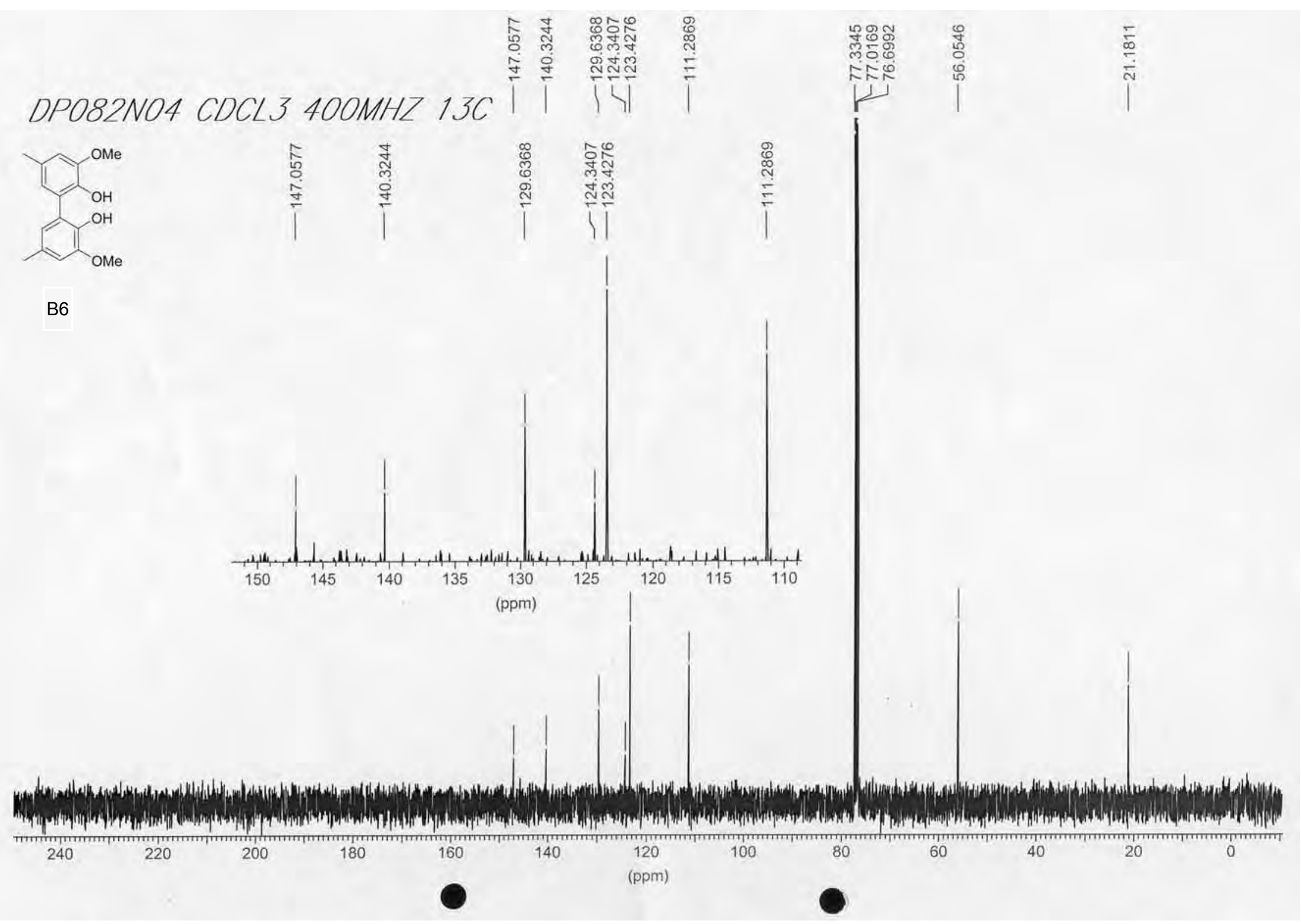




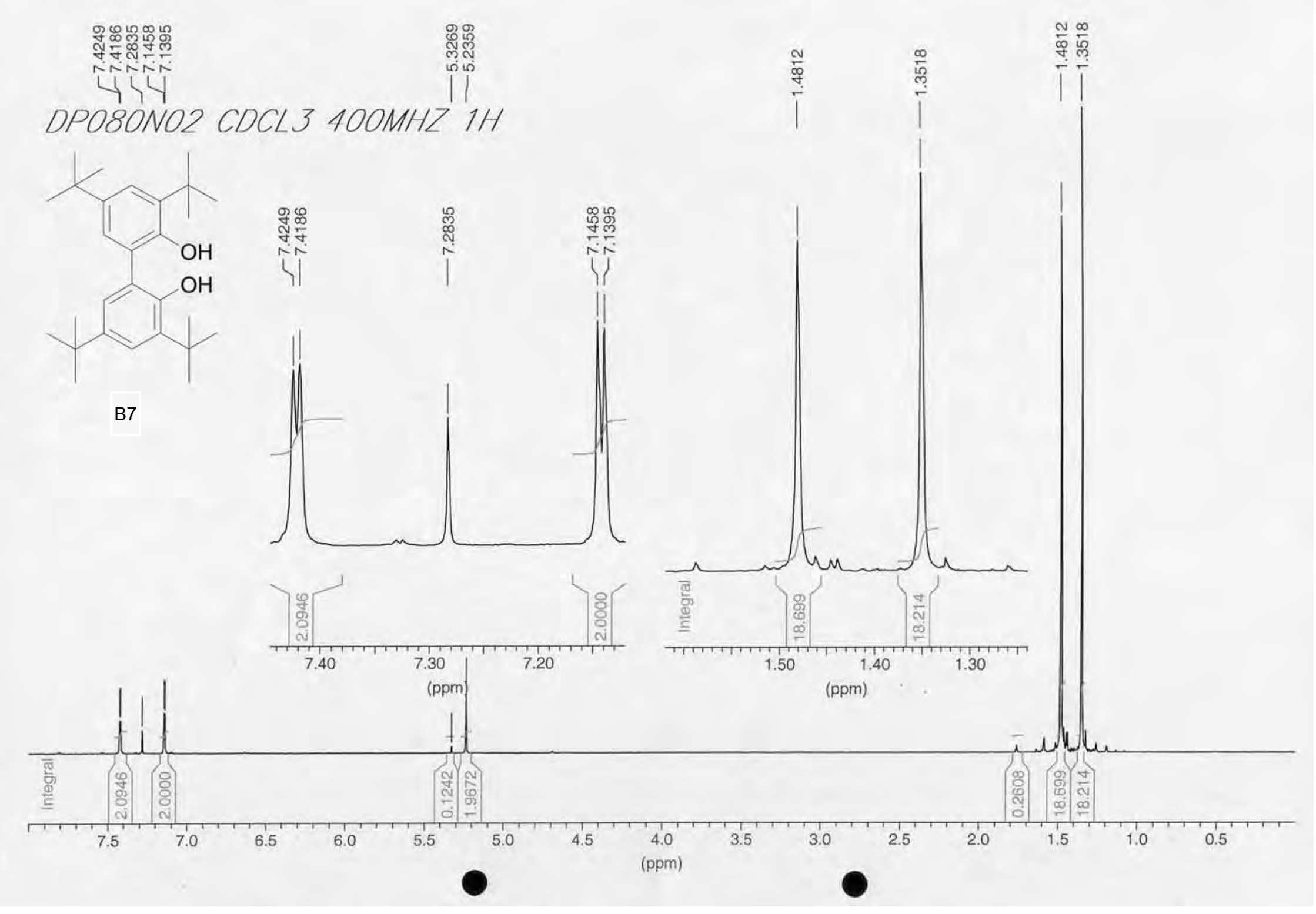




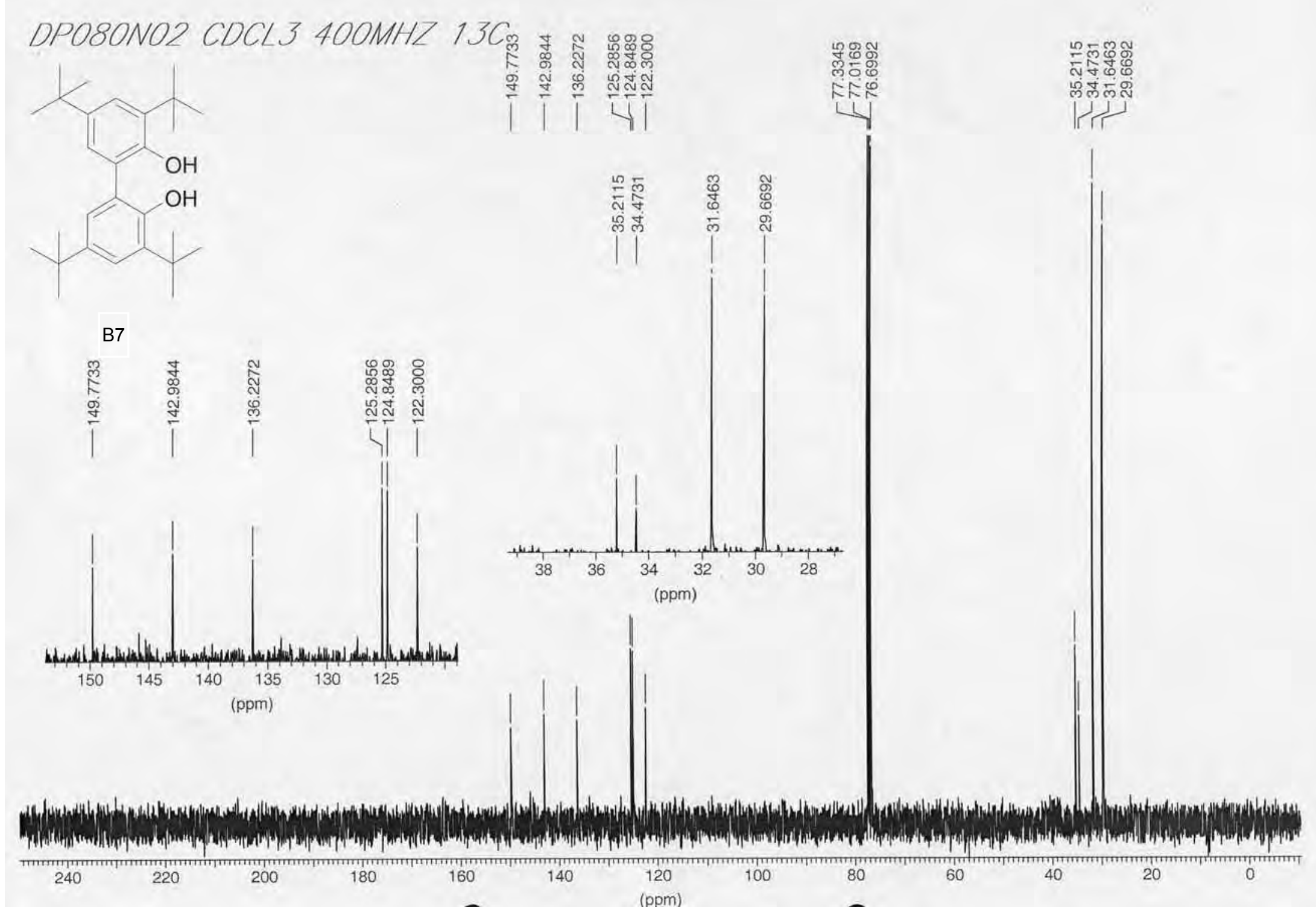




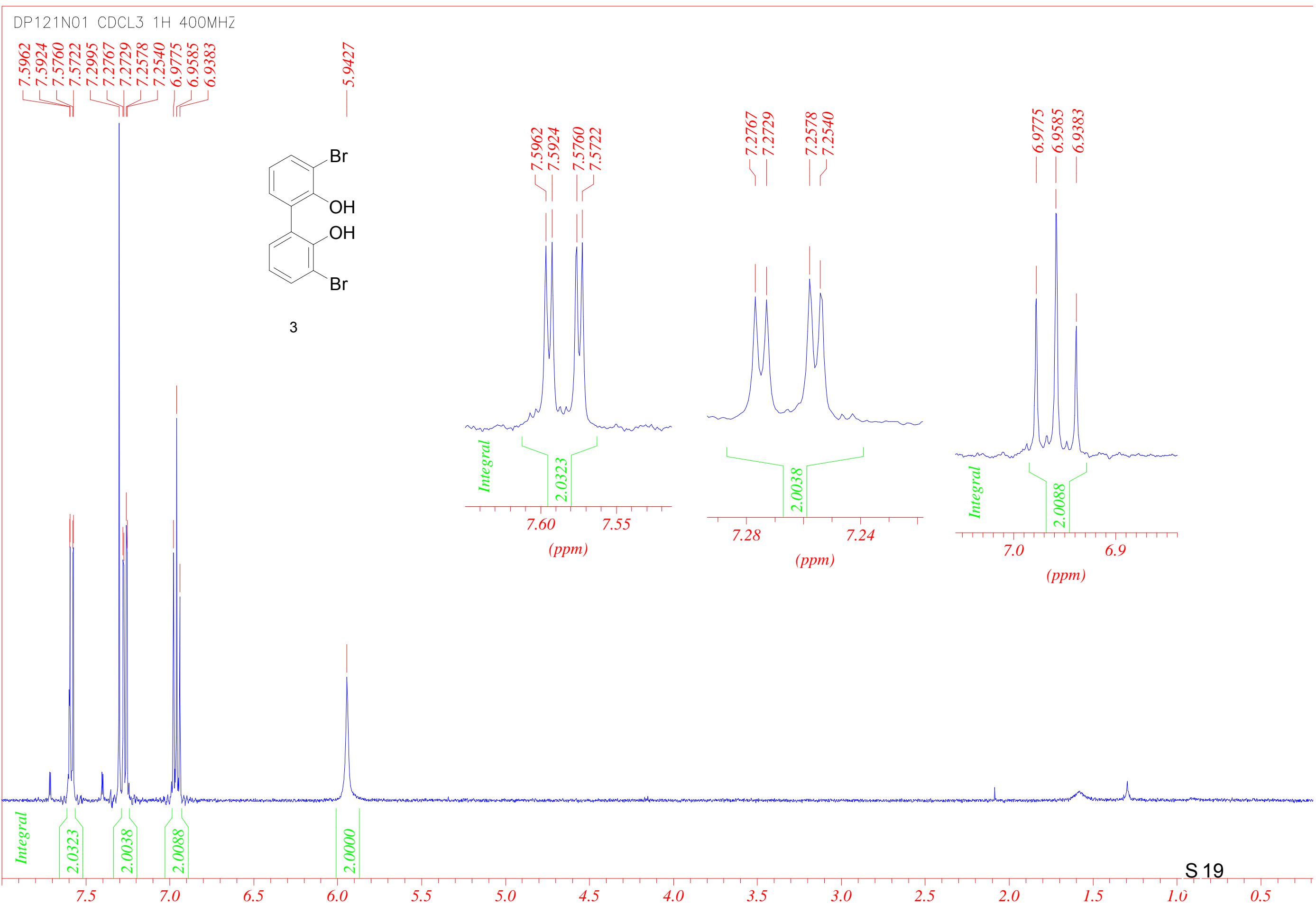




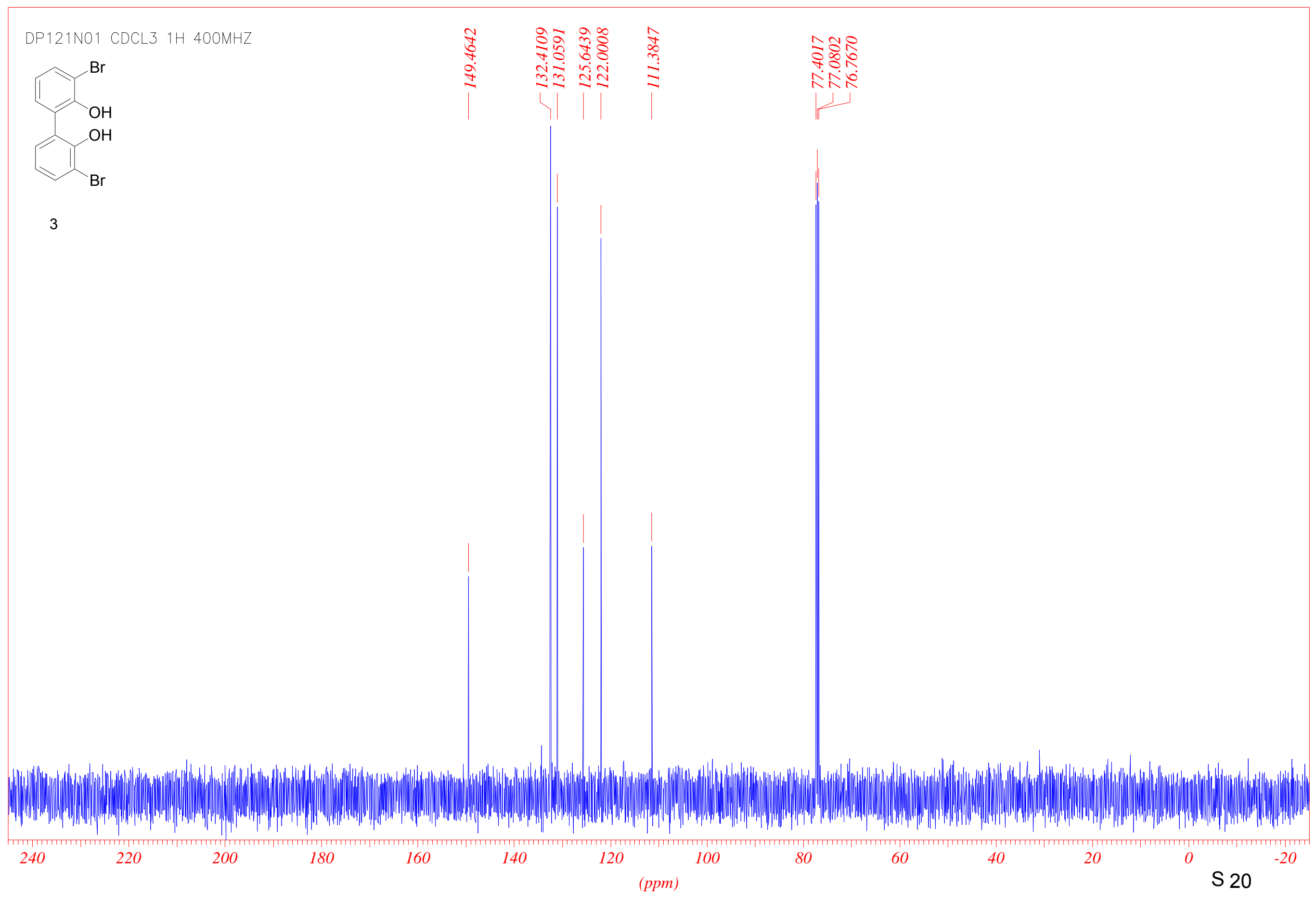


DP123 CDCL3 1H 400MHZ

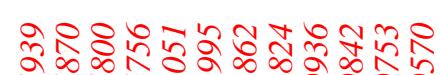

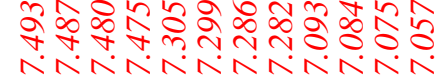

LiL L L L

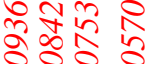

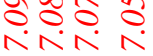

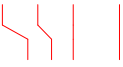

B8
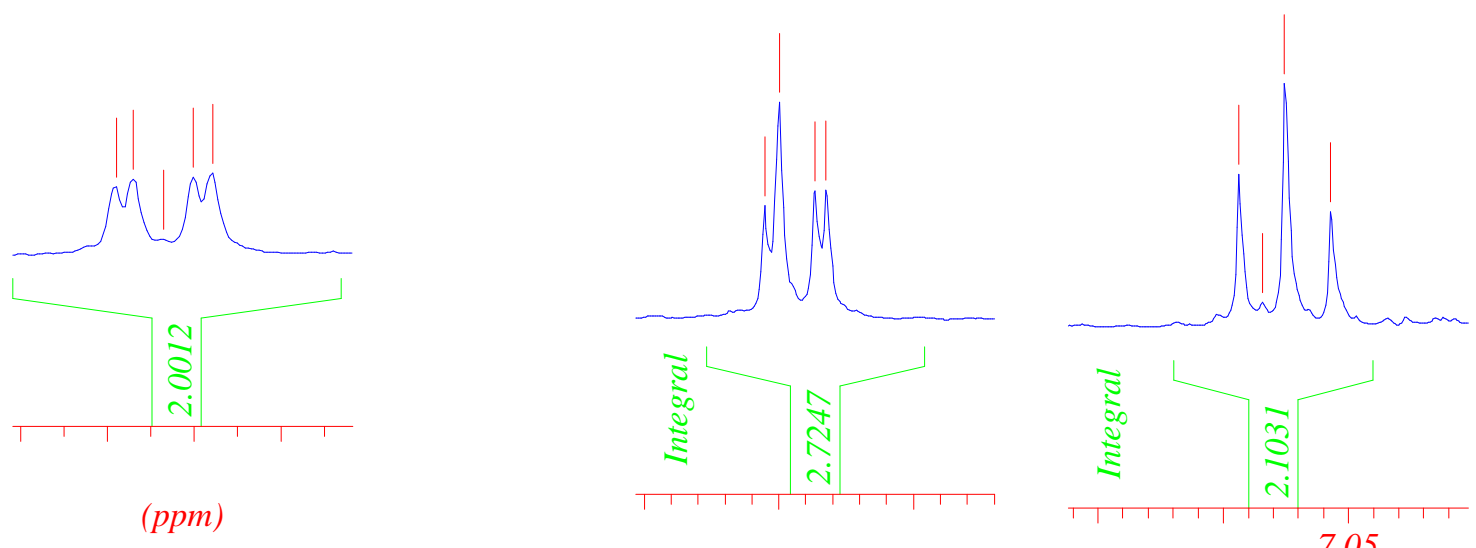

(ppm)

(ppm)

(ppm)

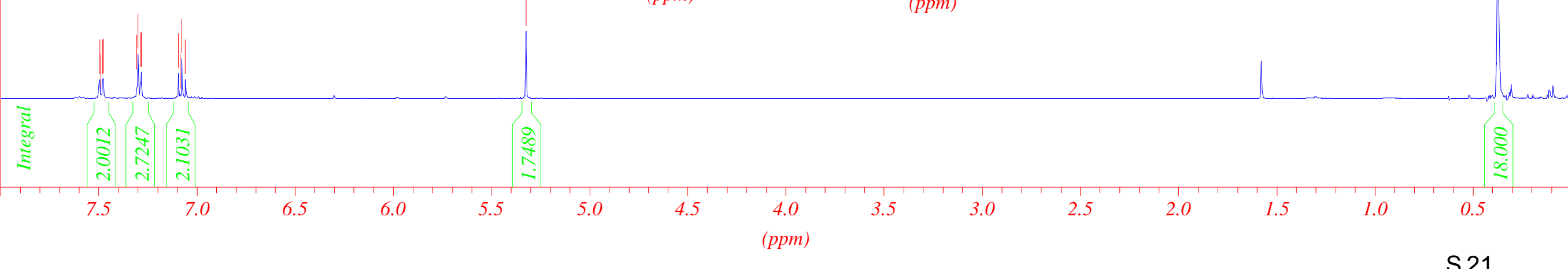




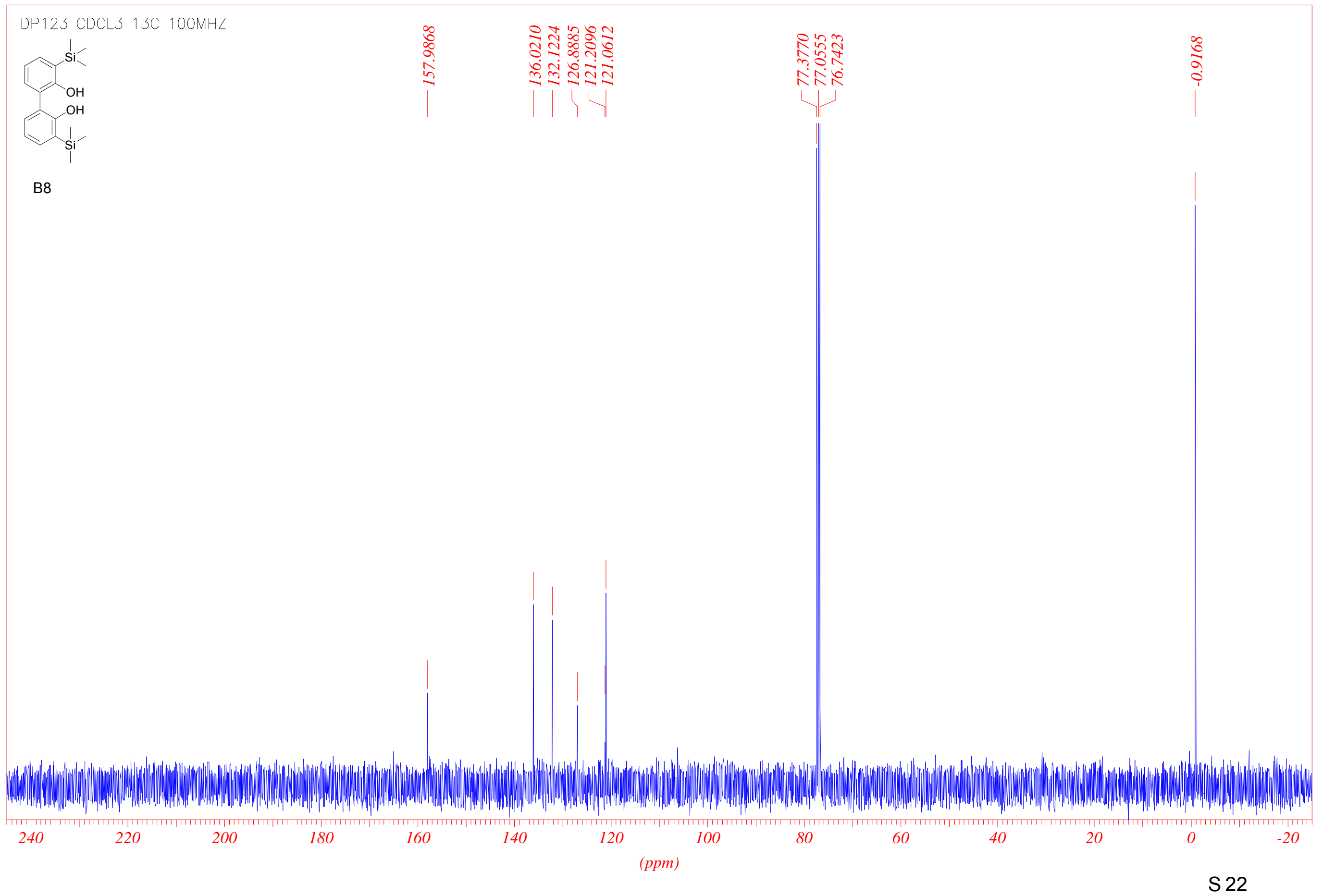




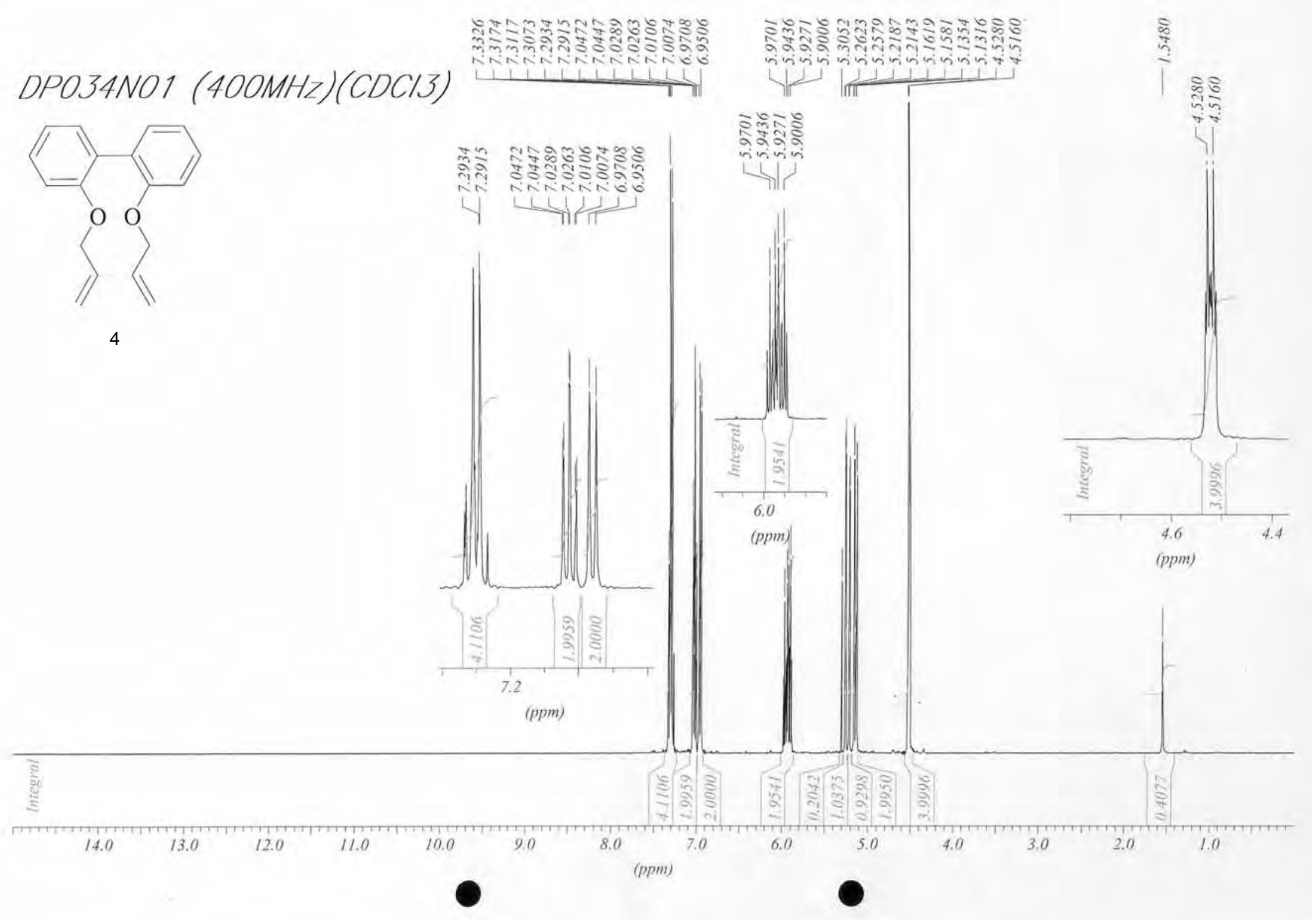




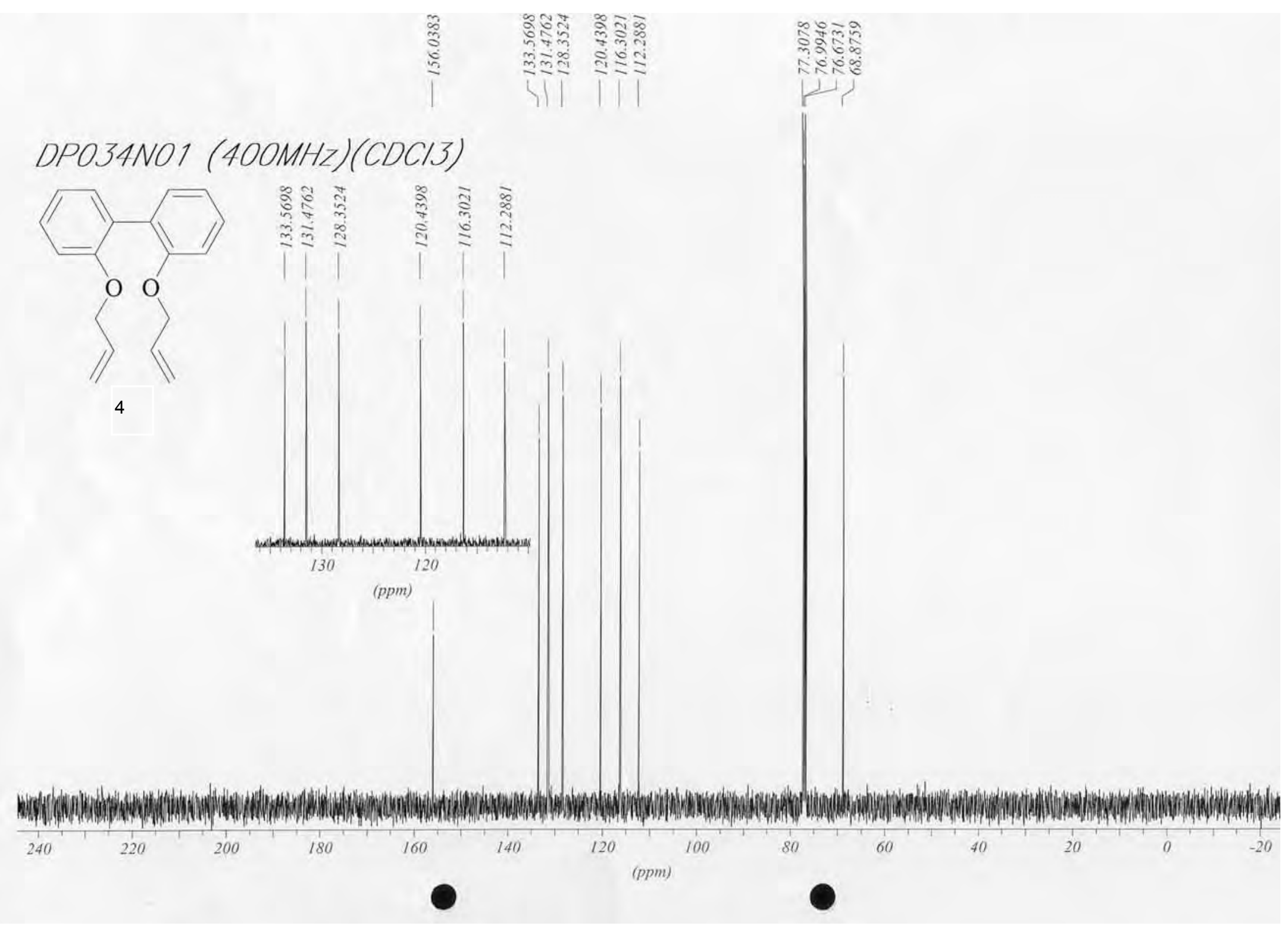




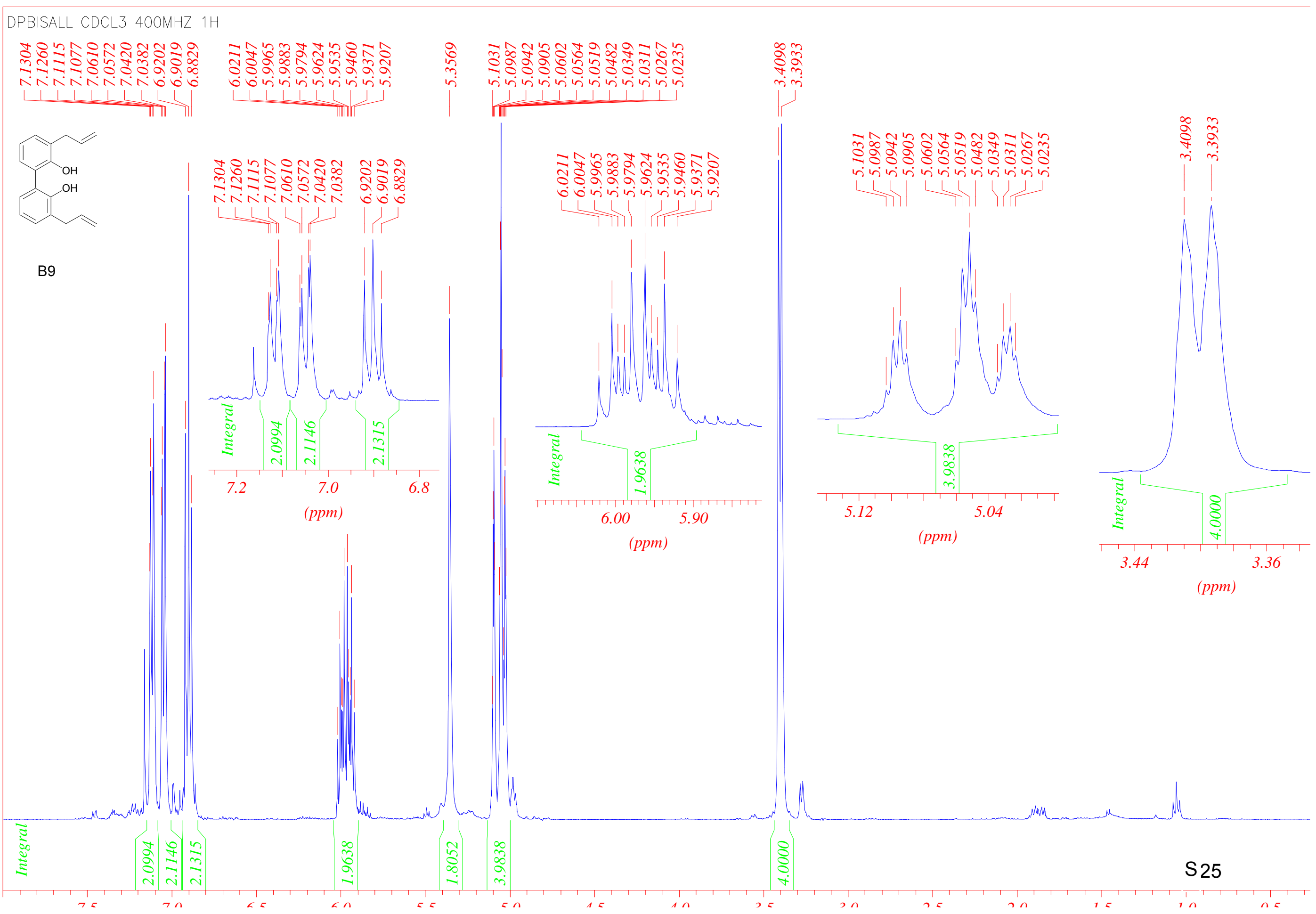




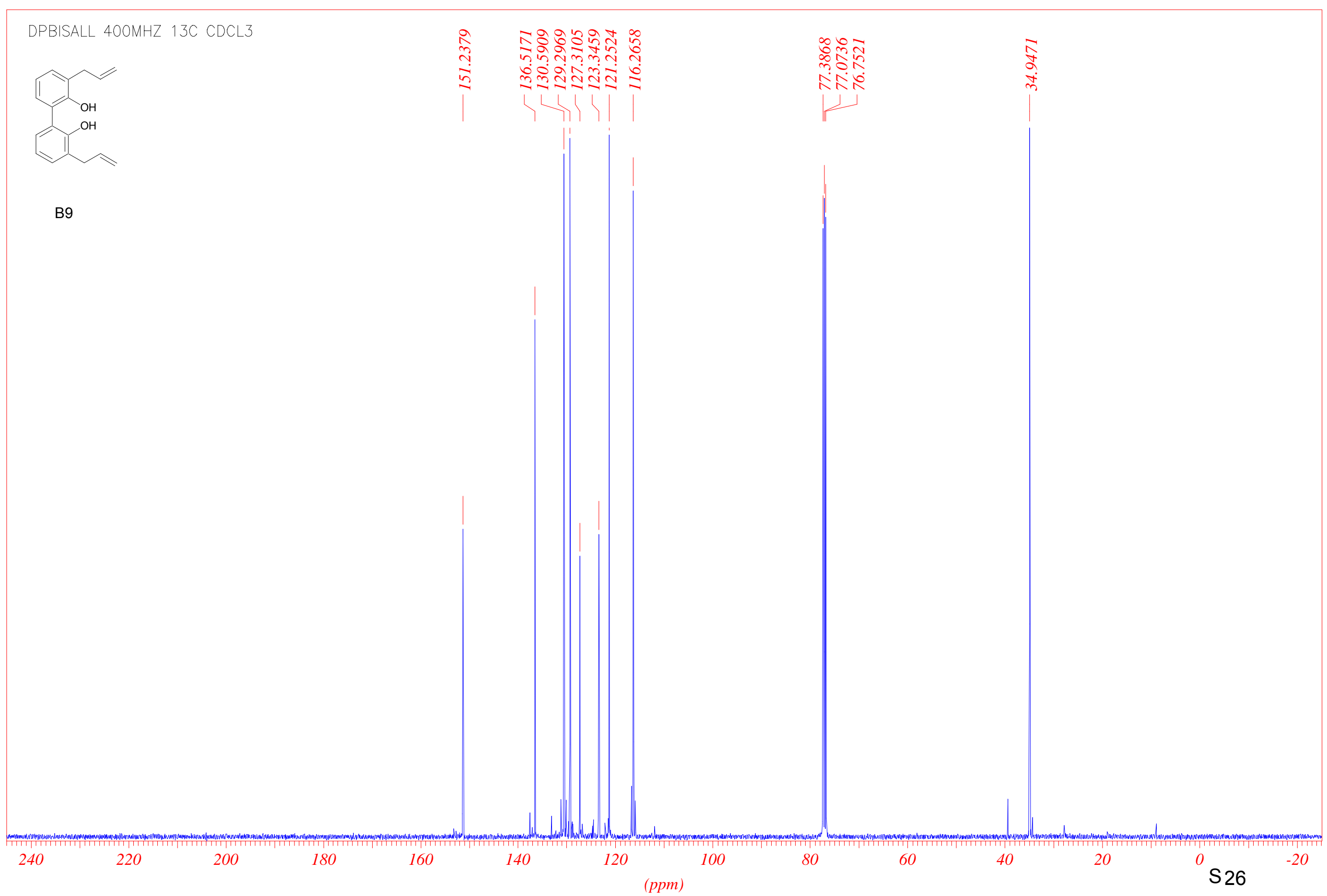




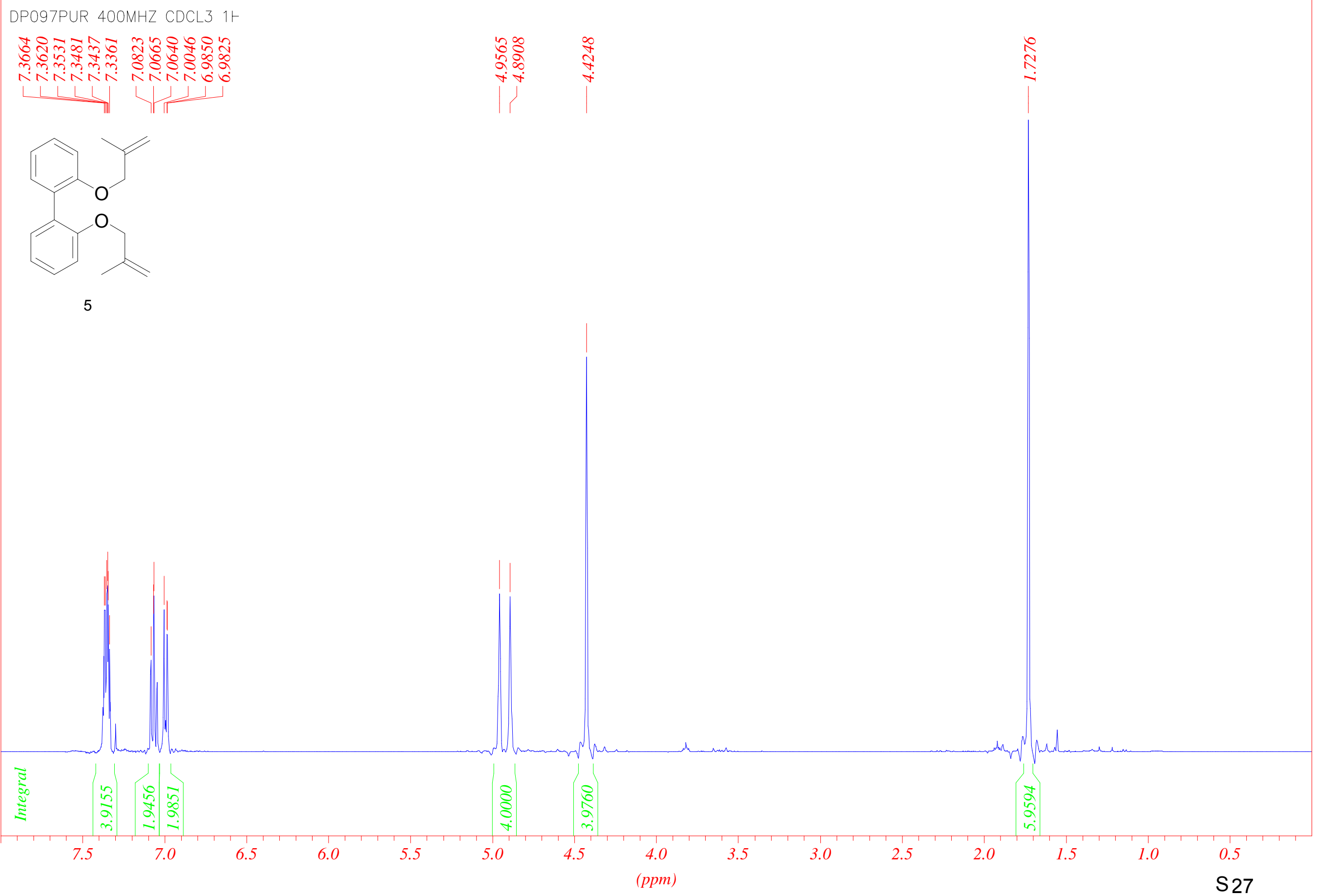




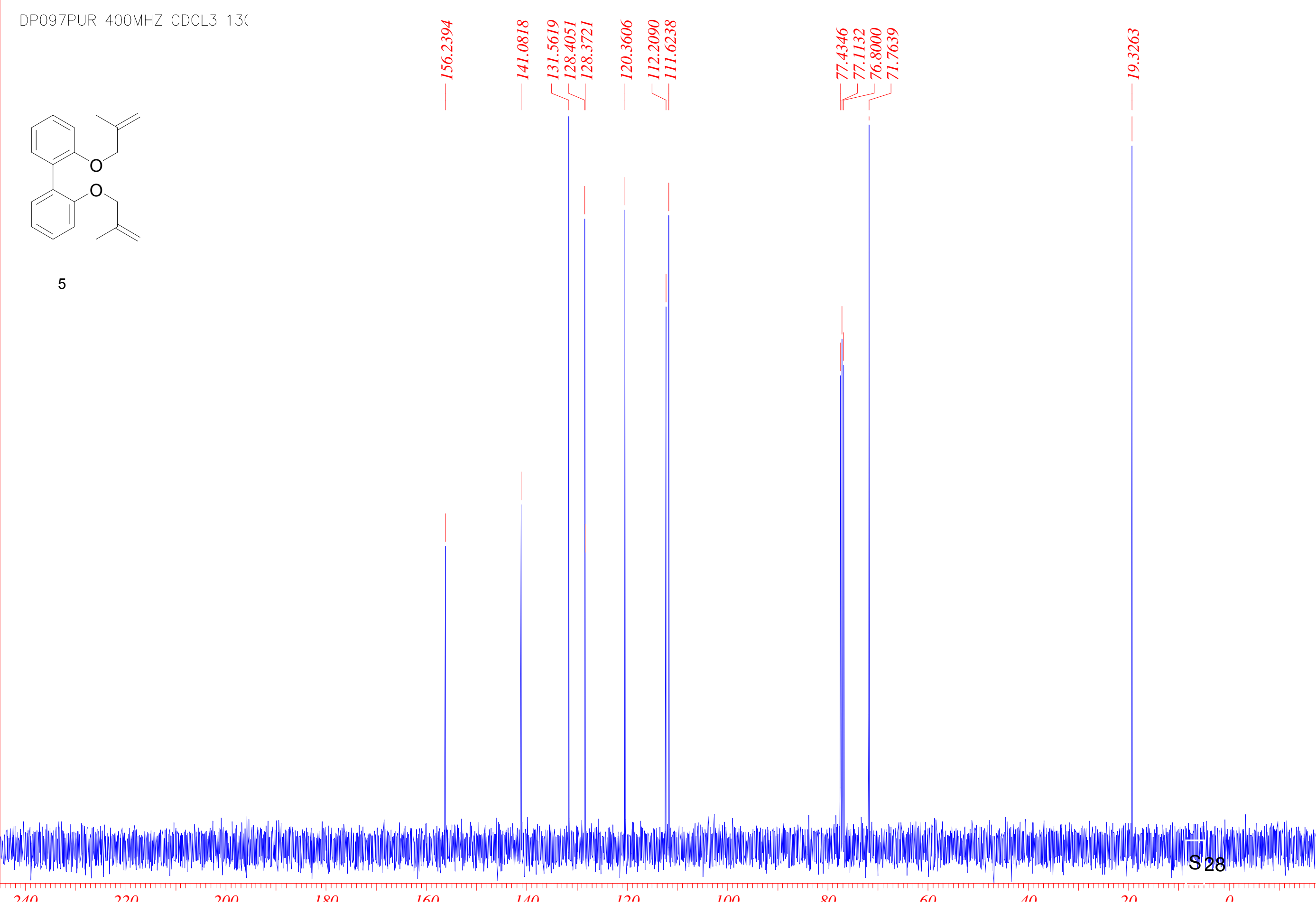




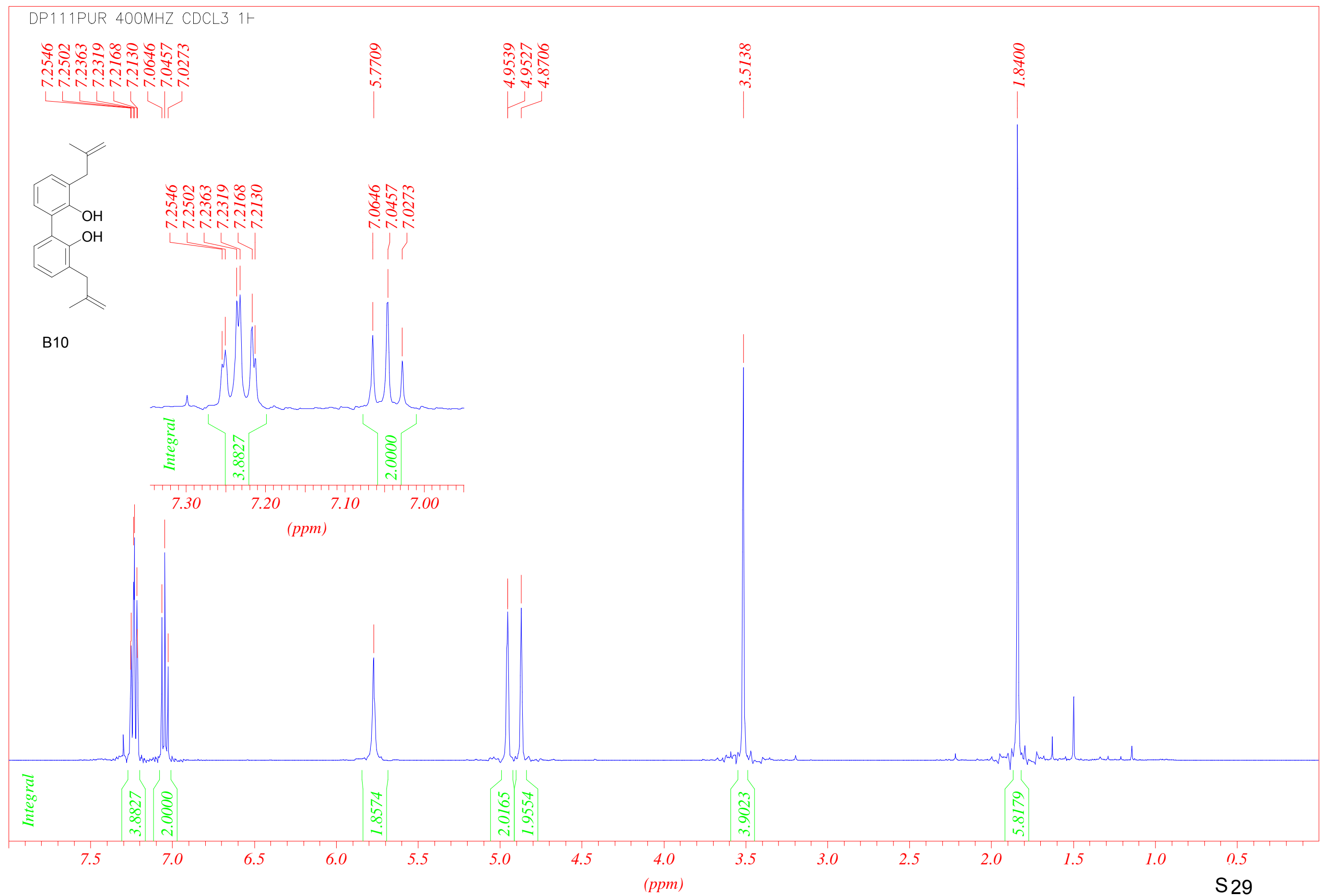




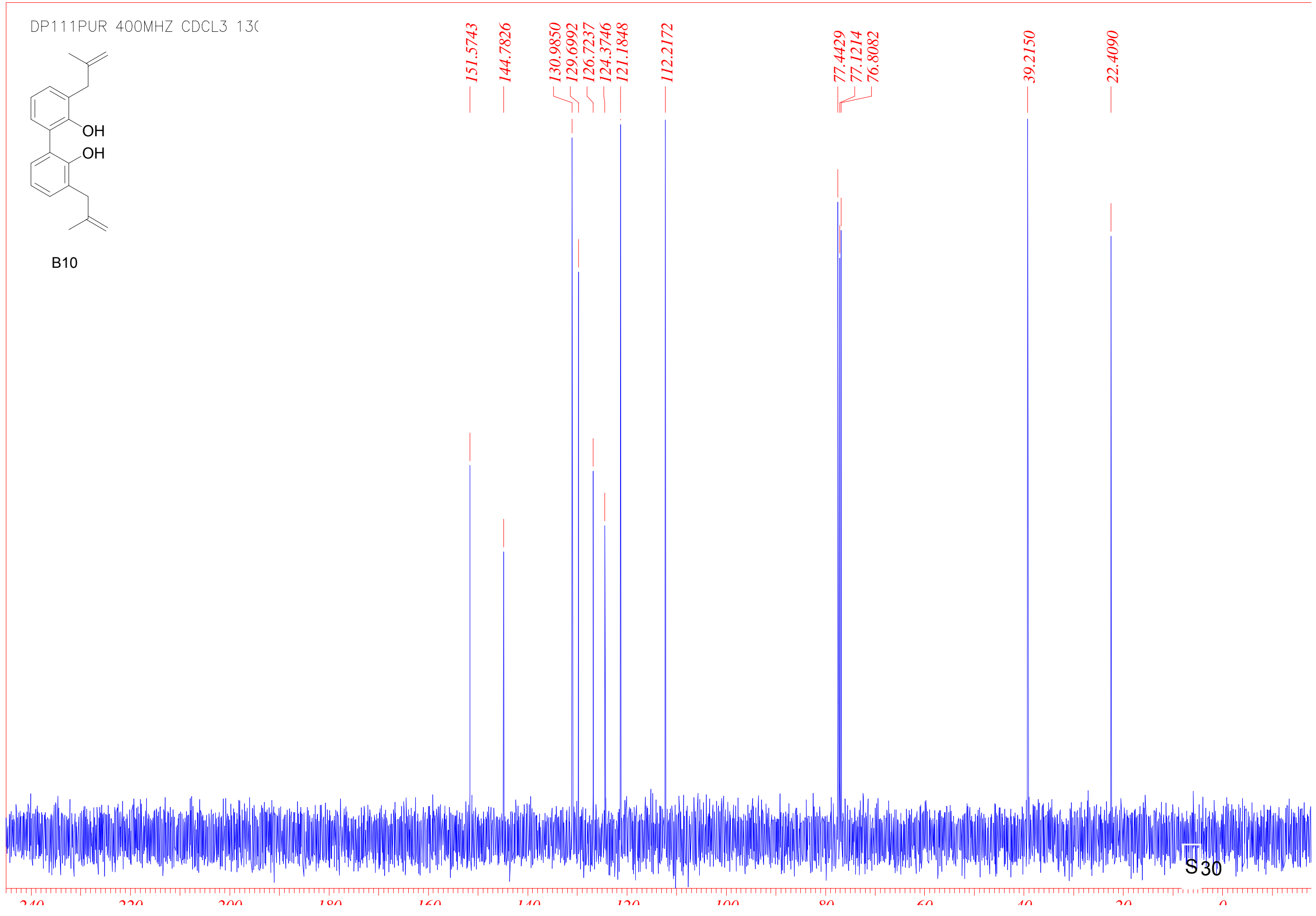




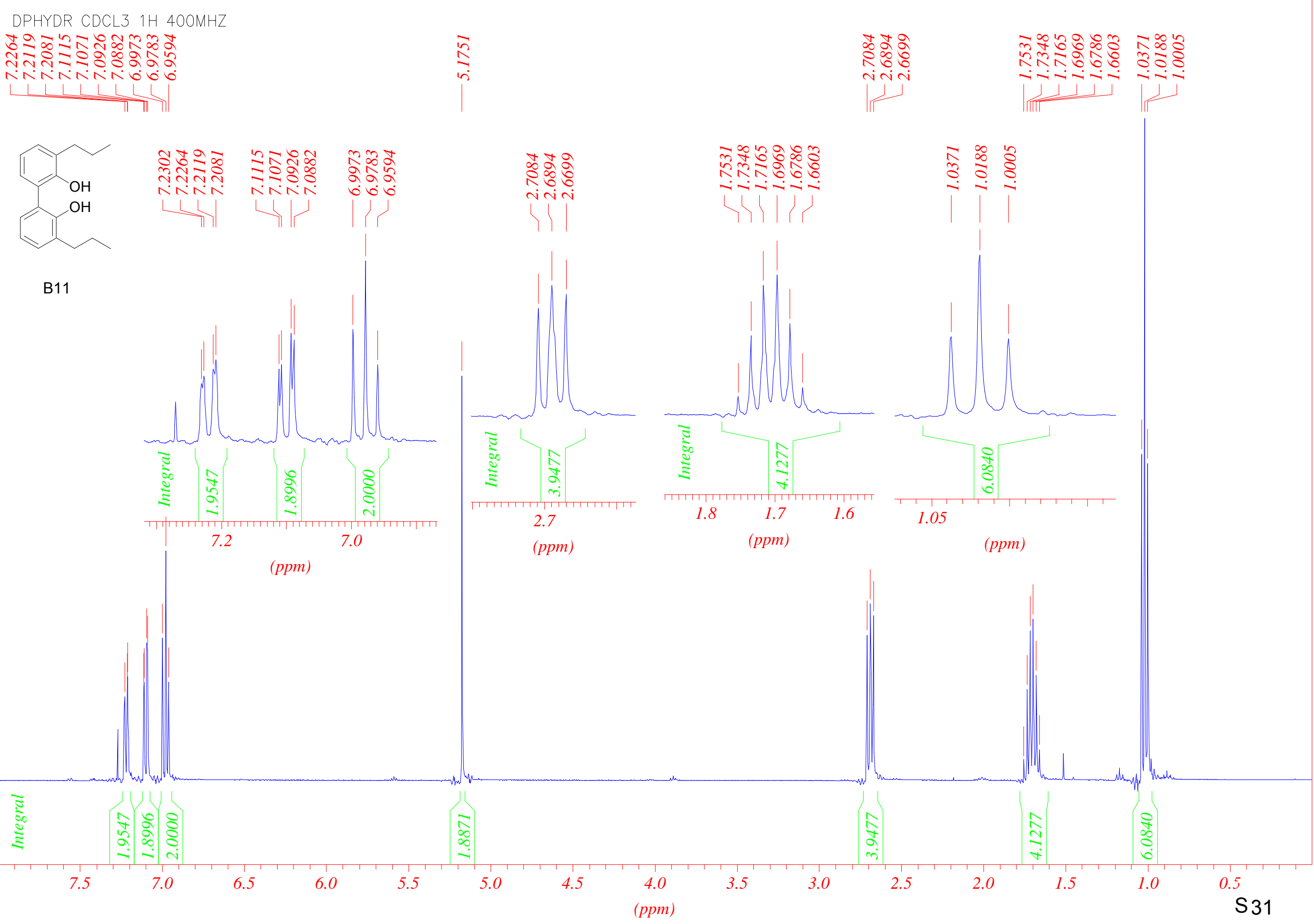




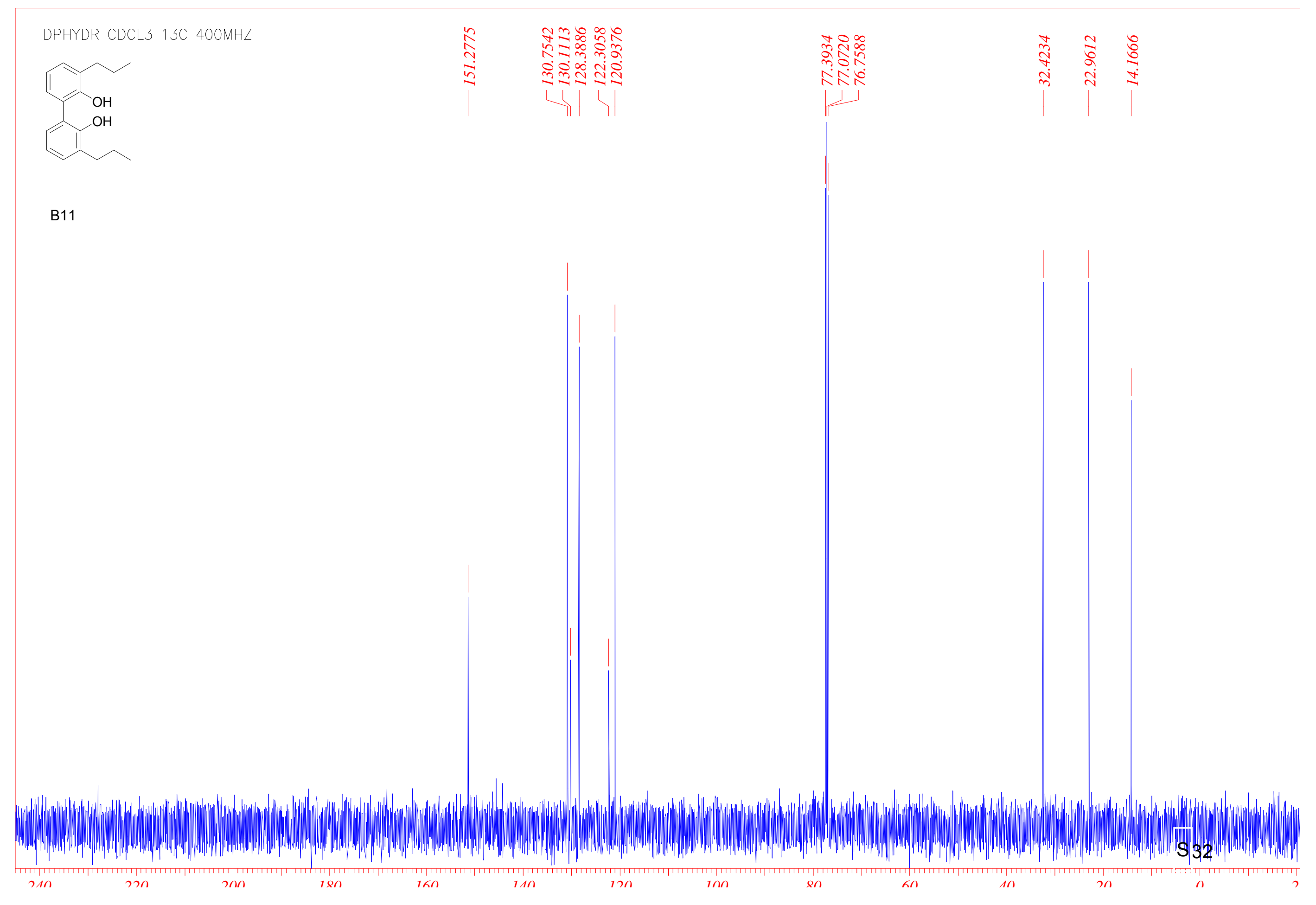




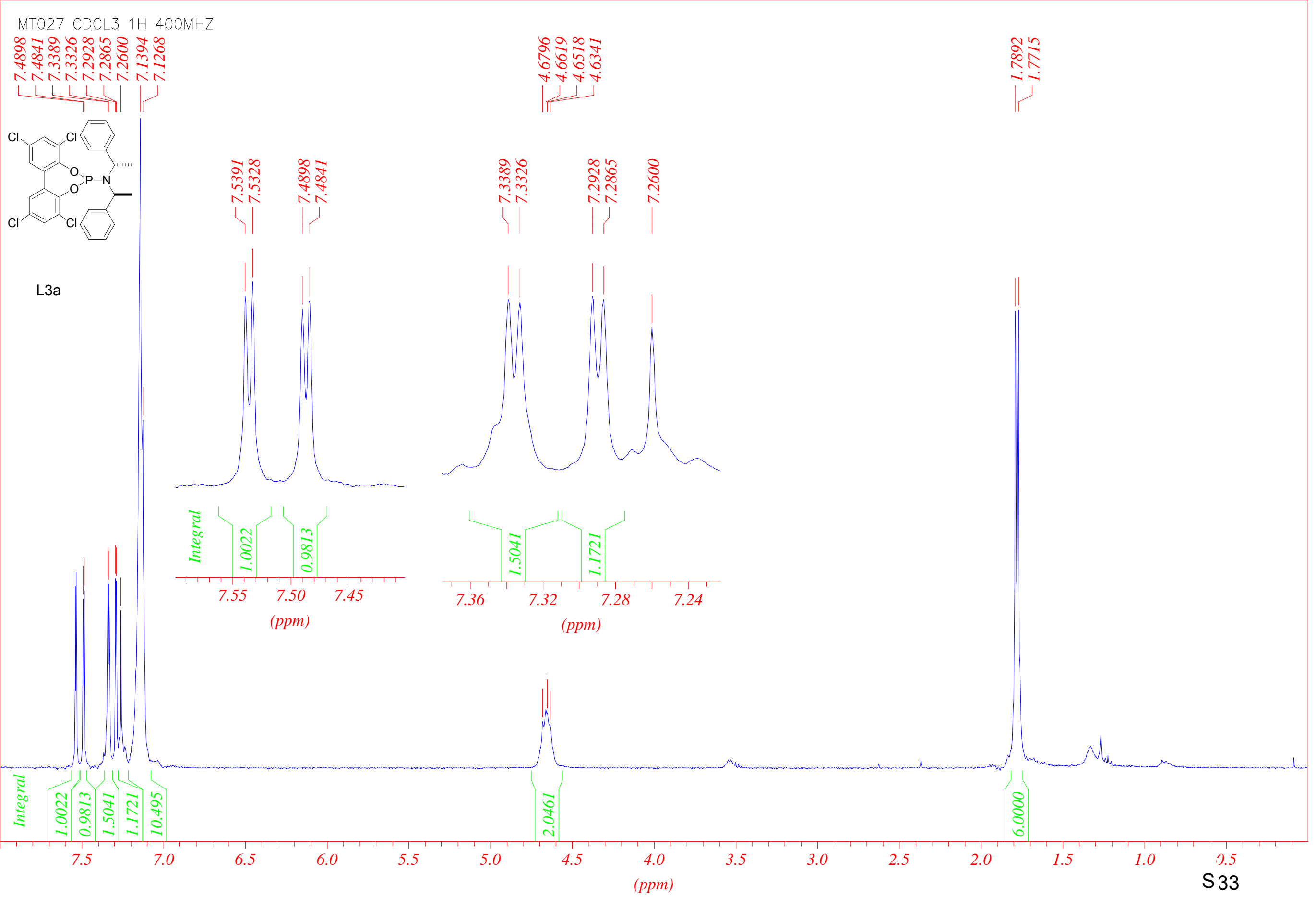




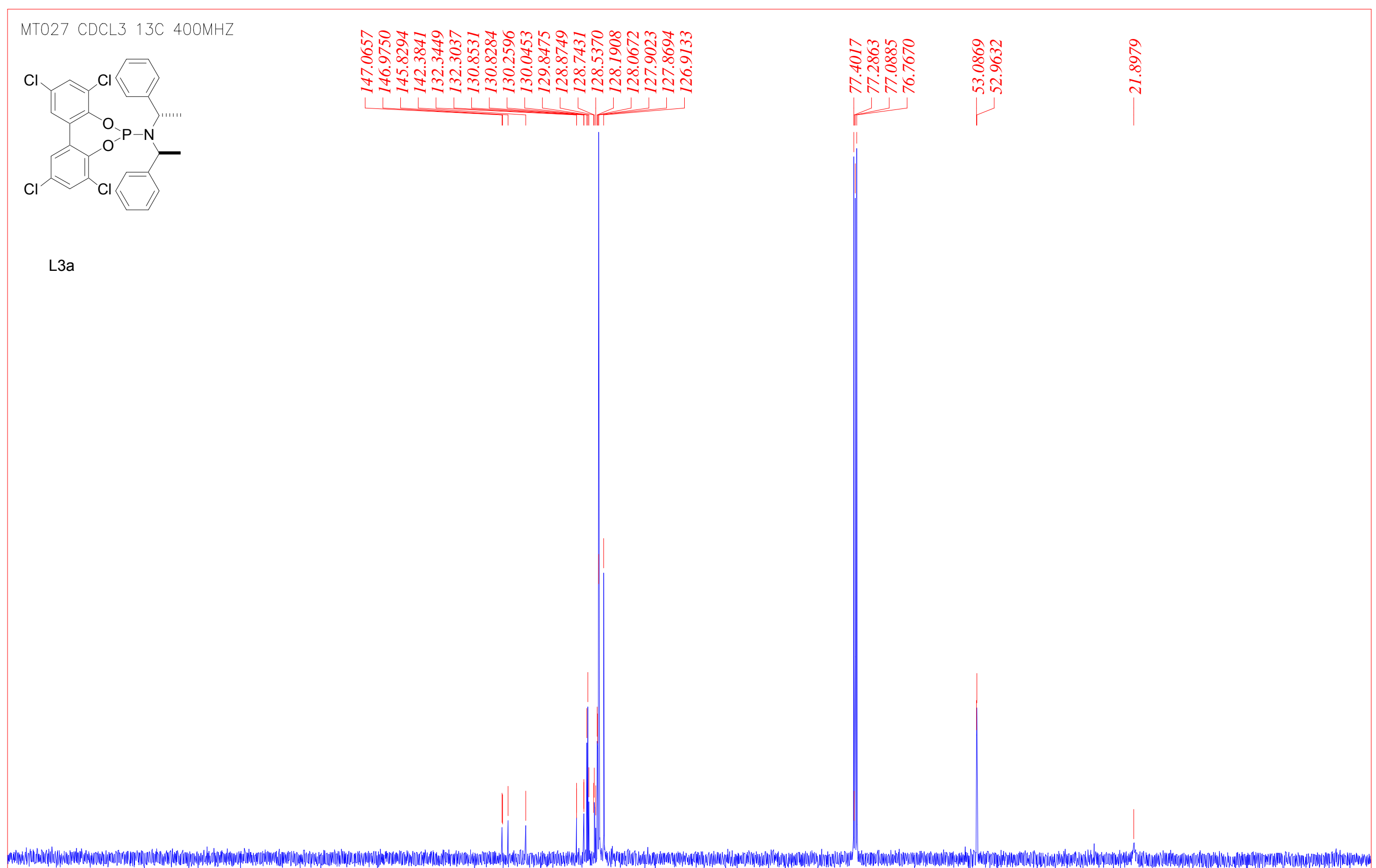

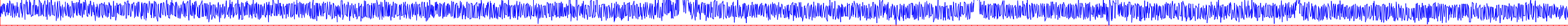

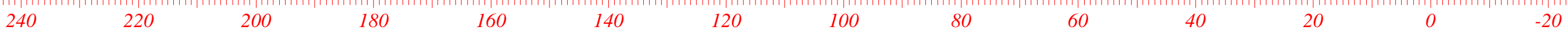




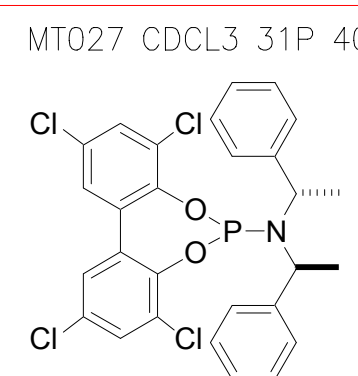

L3a

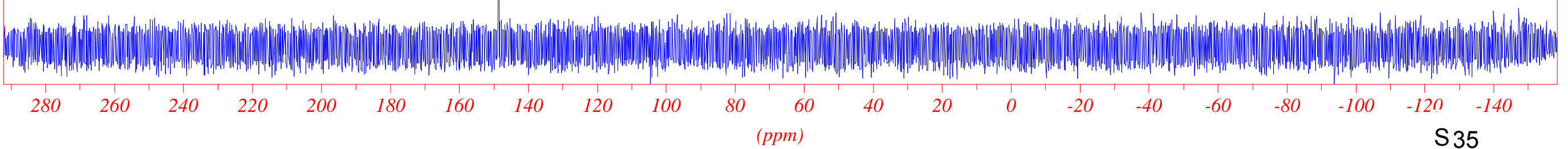




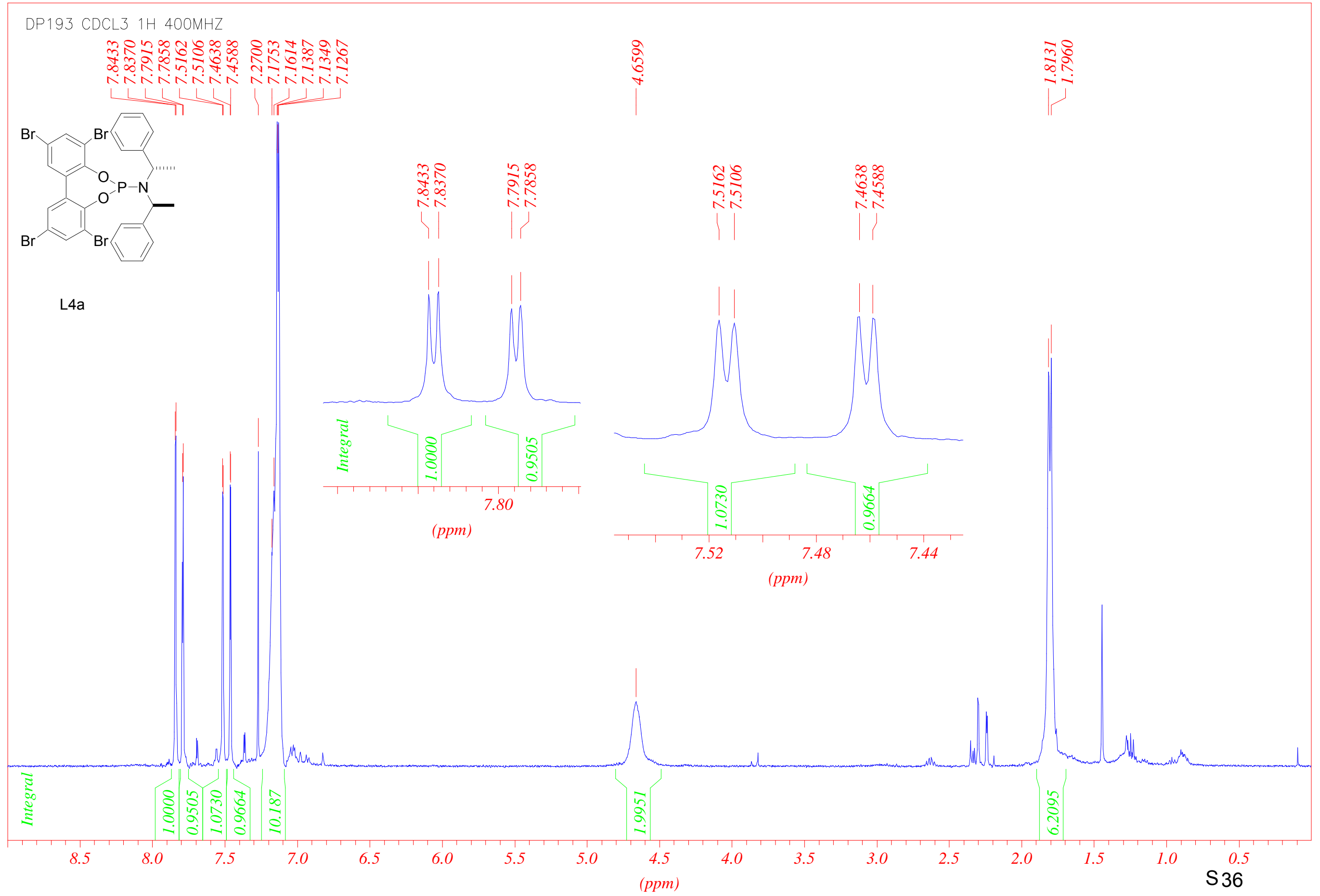




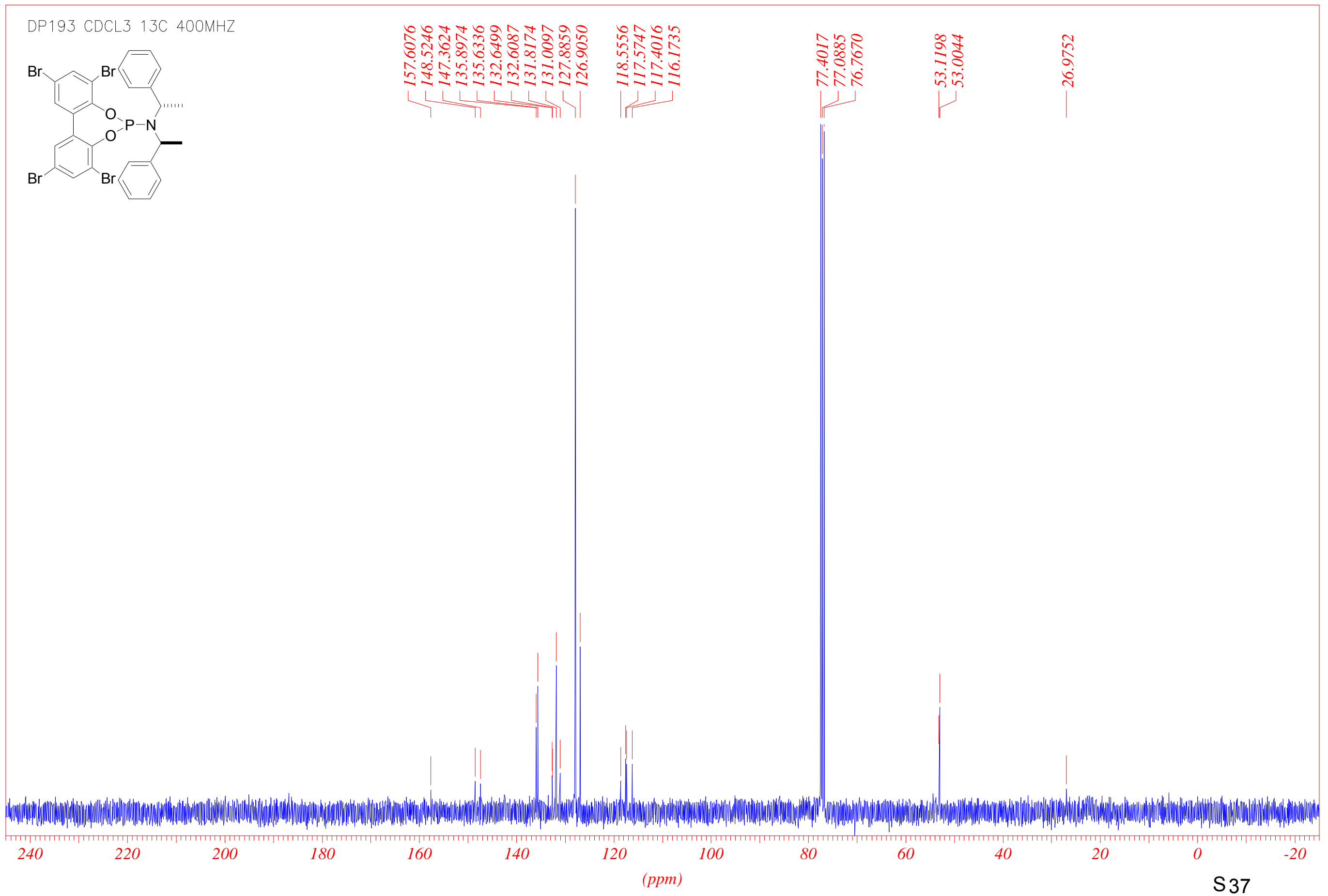




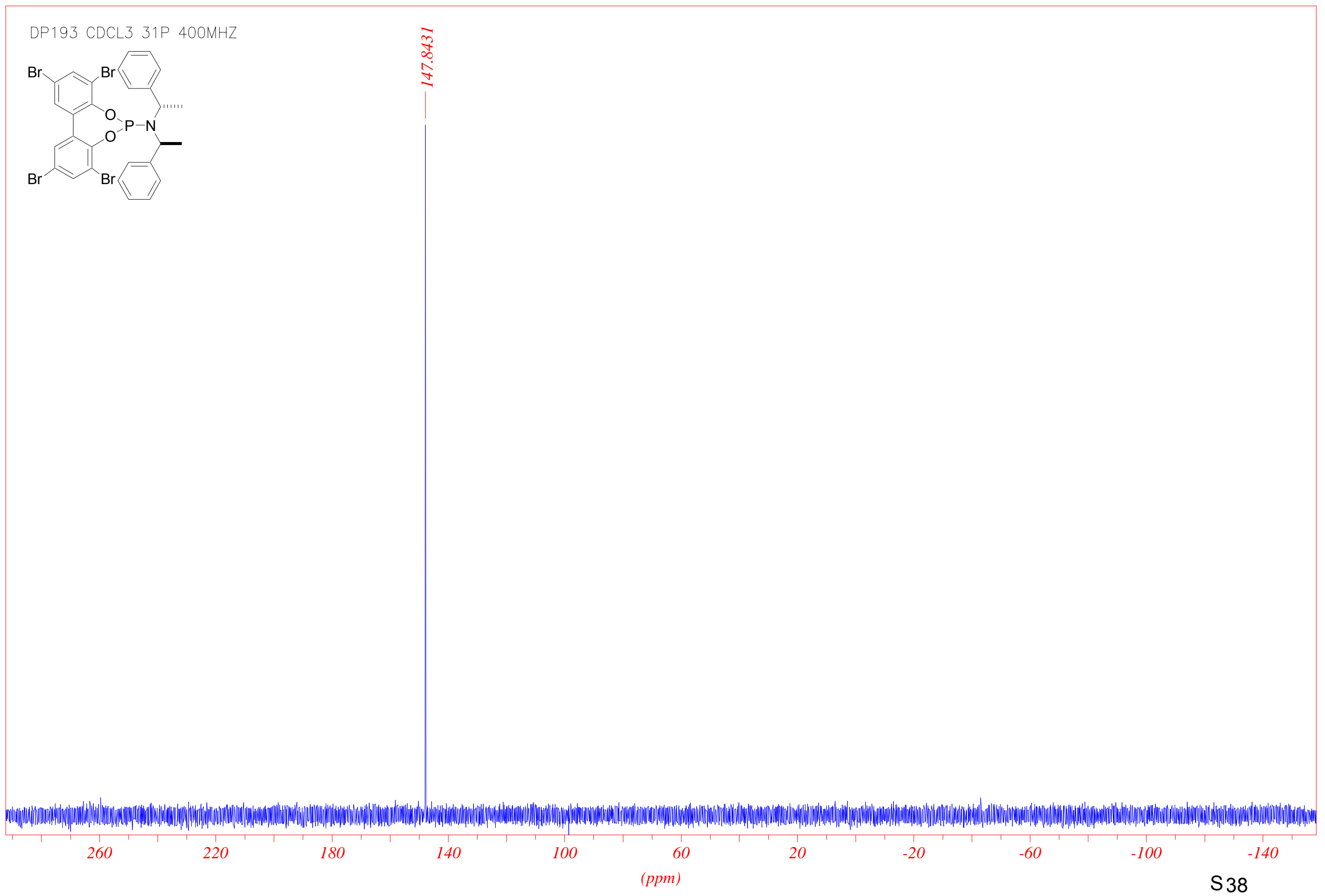




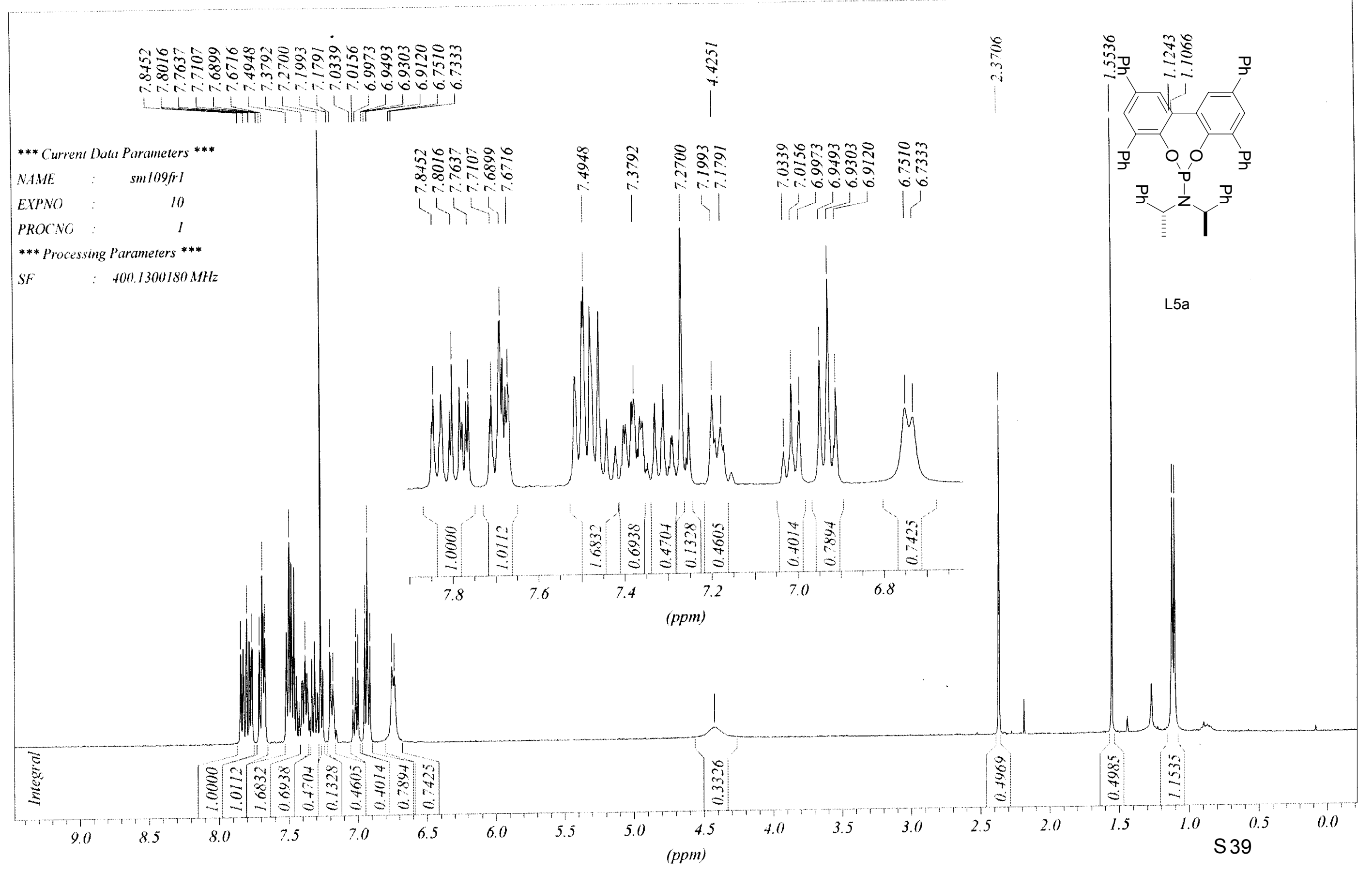




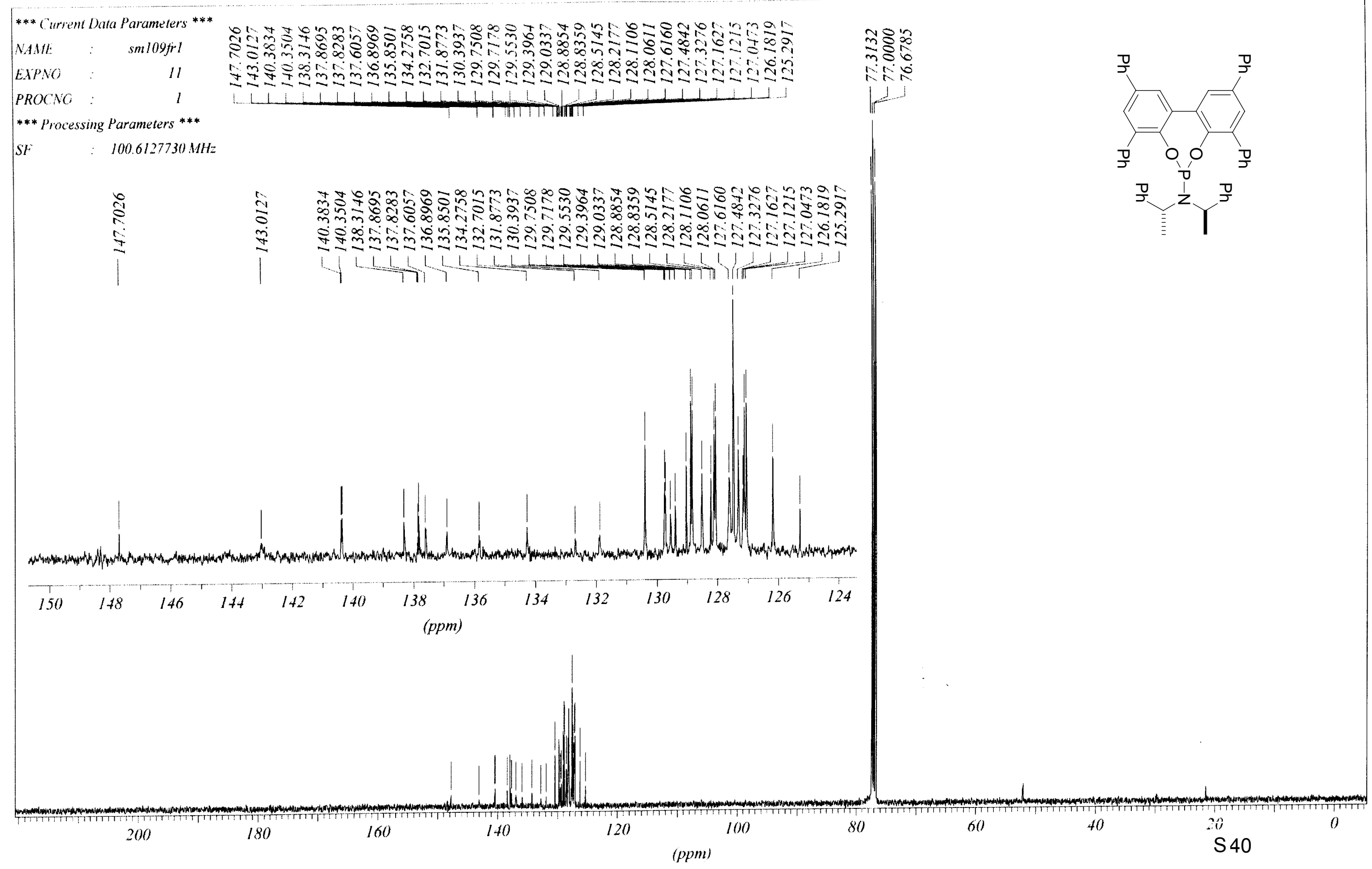




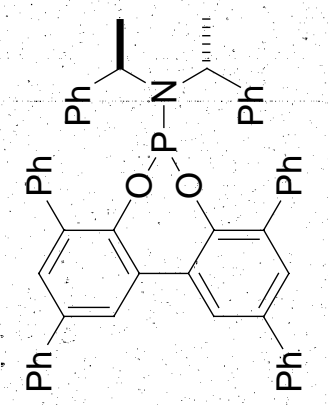




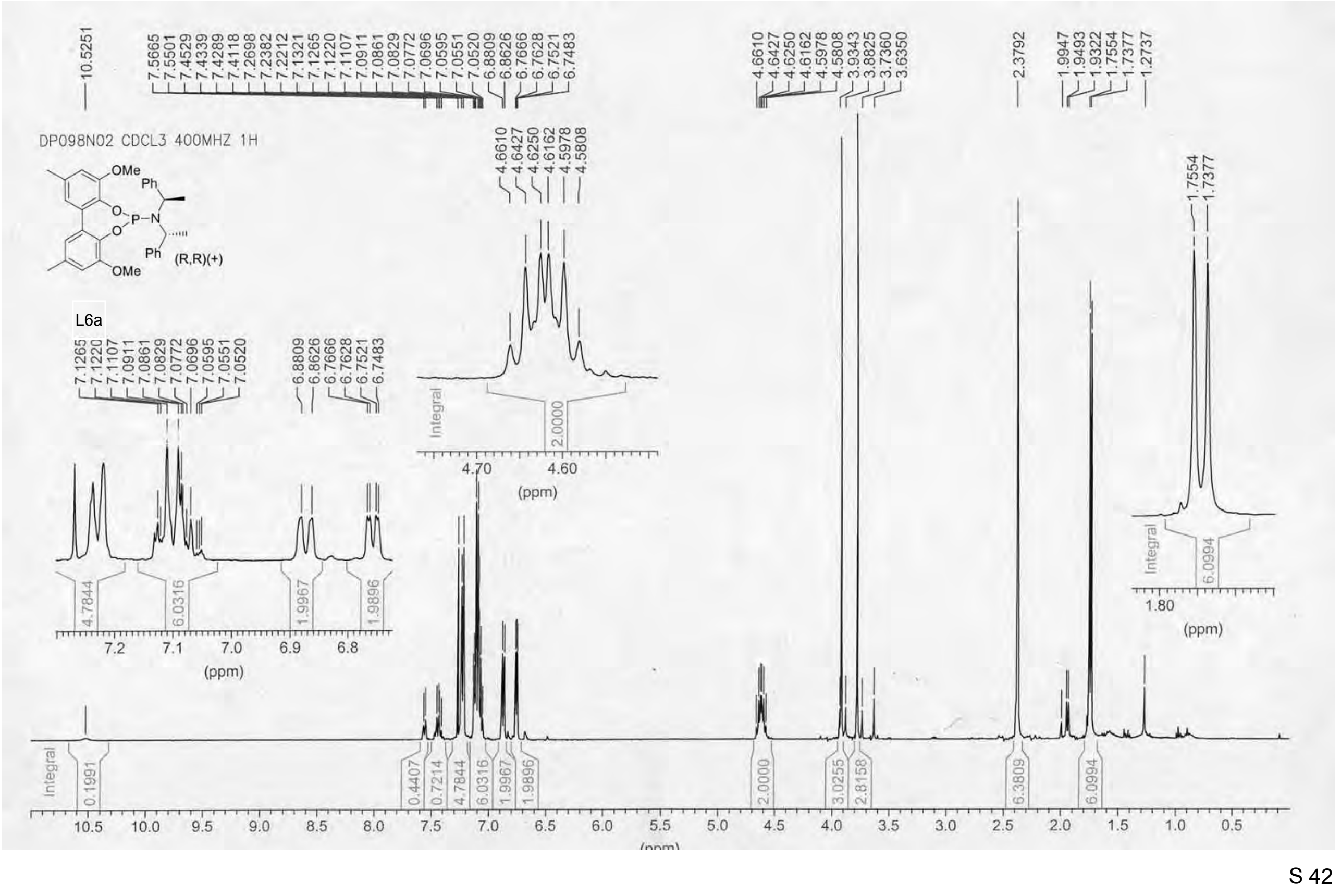


DP098NO2 CDCL3 400MHZ 13C
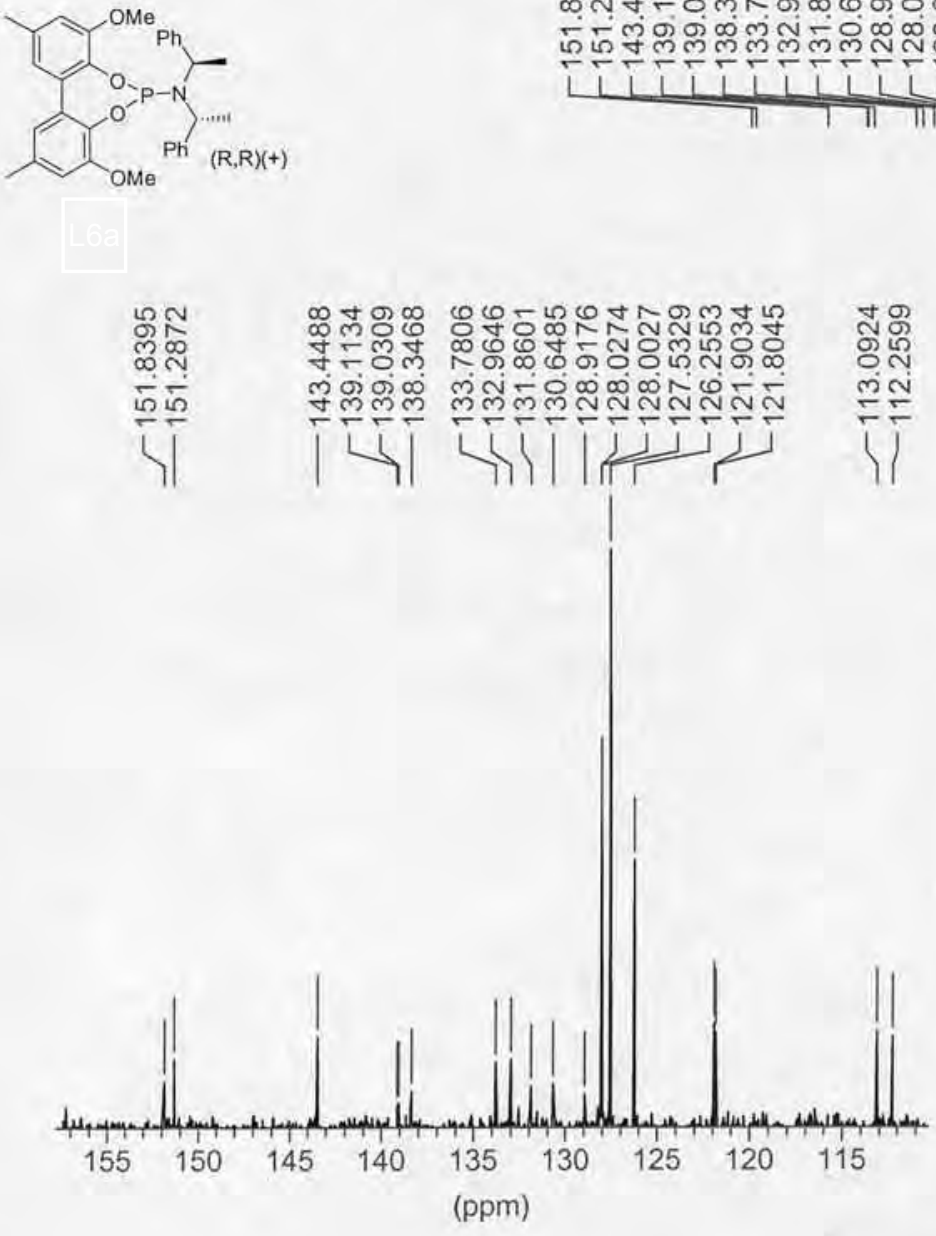

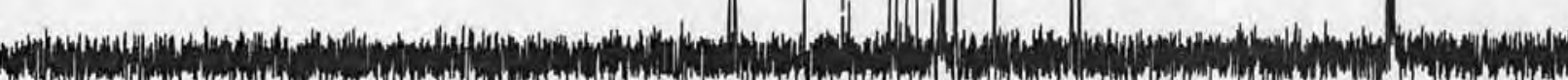

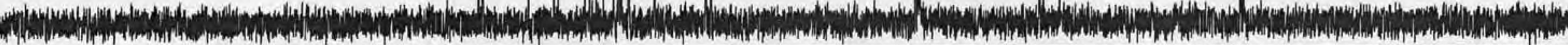

210

190

170

150

130

110

90

10

$-30$ 


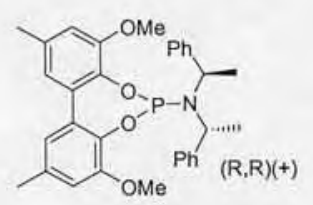




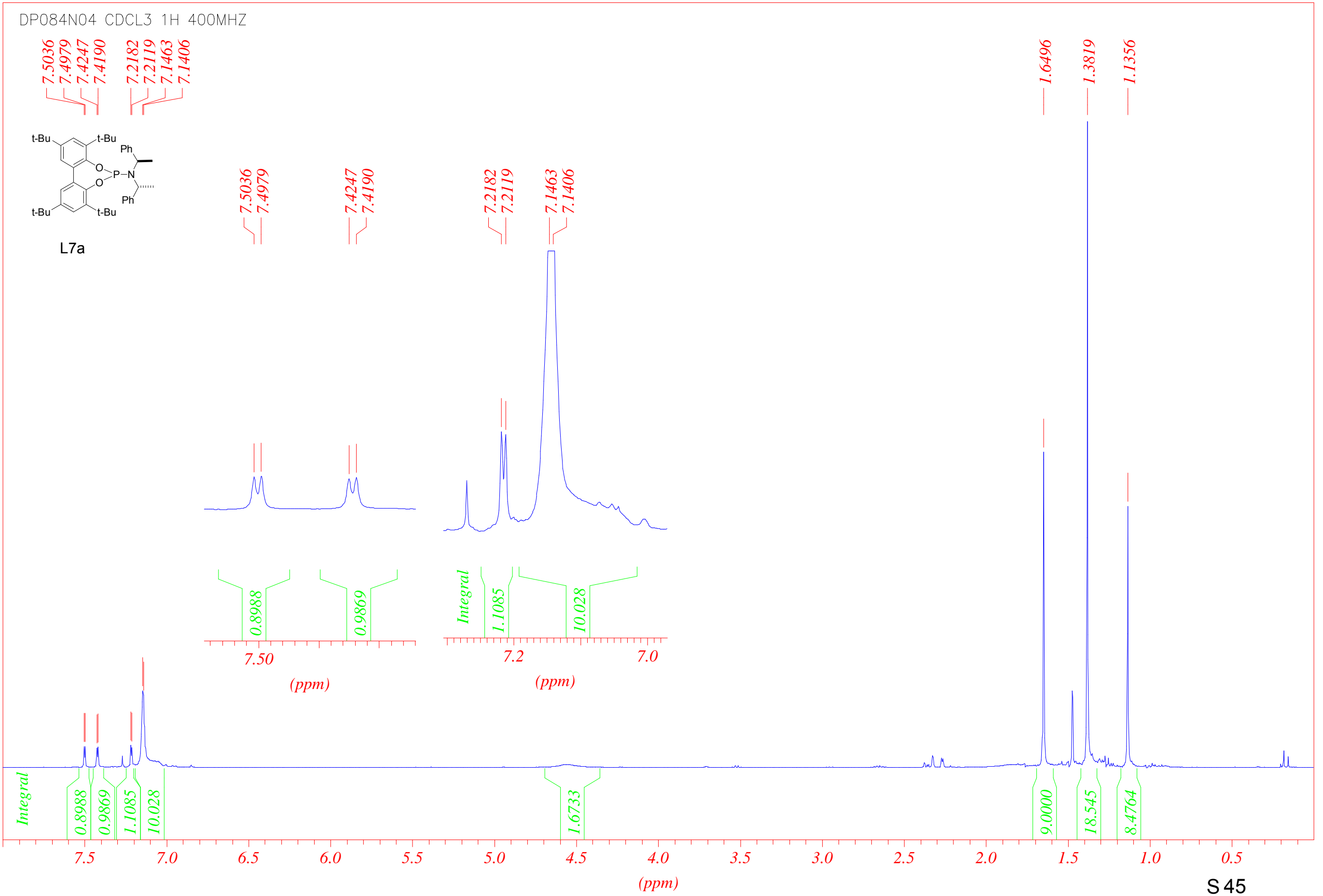




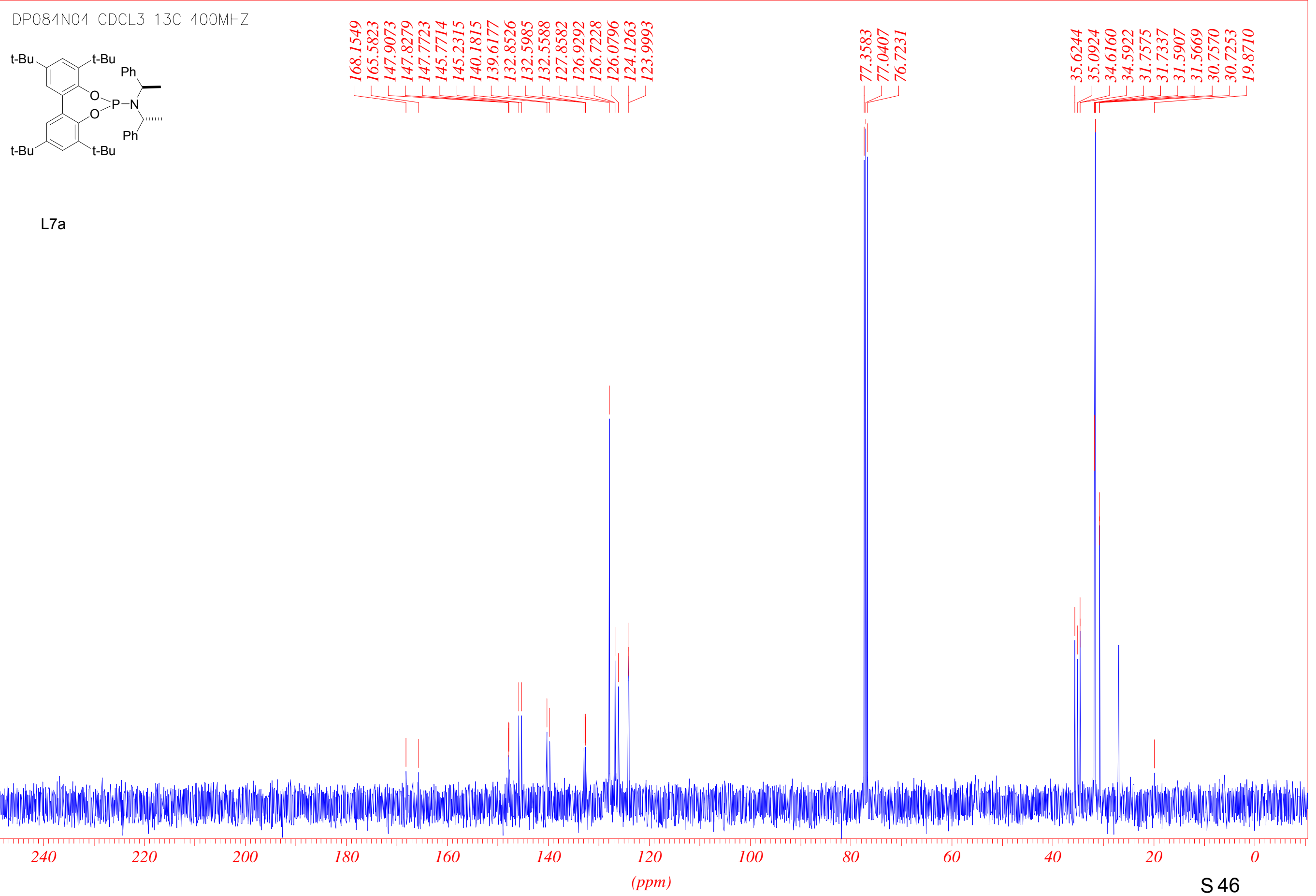




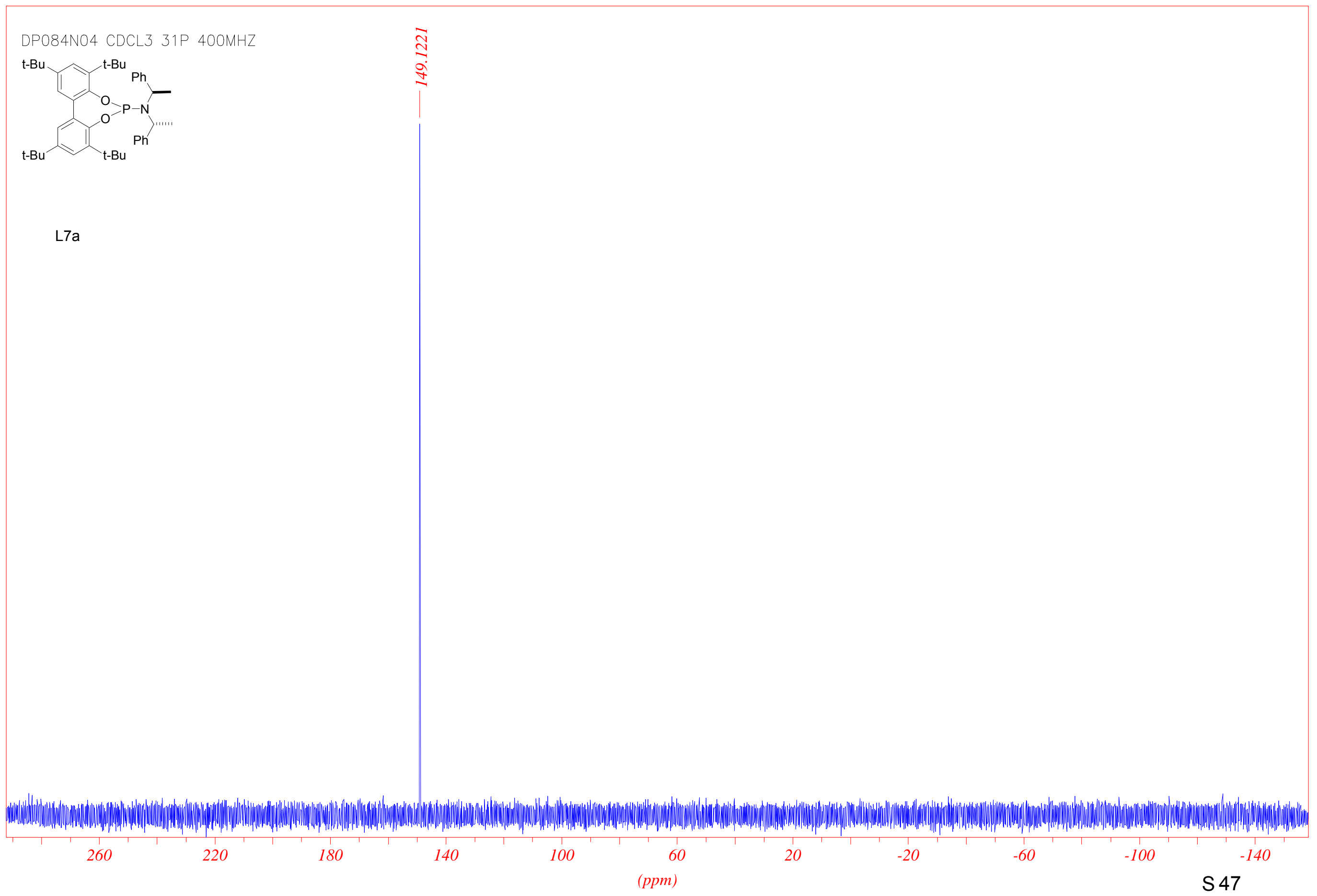



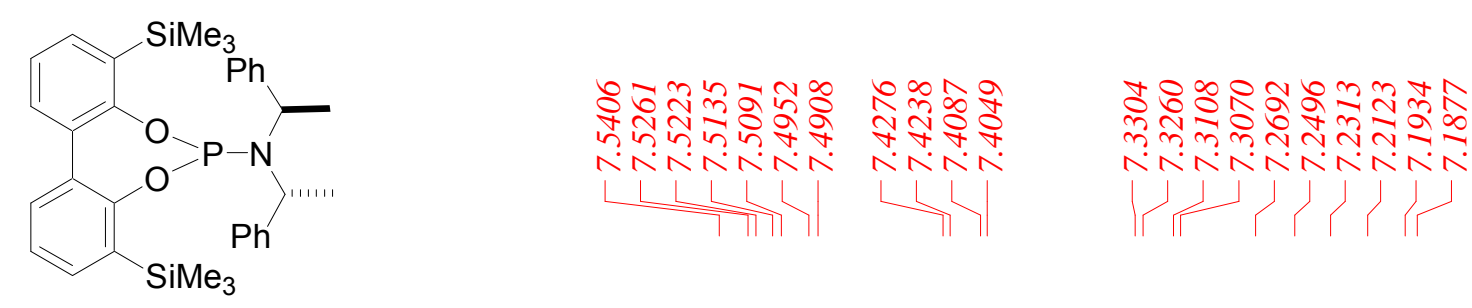

L8a

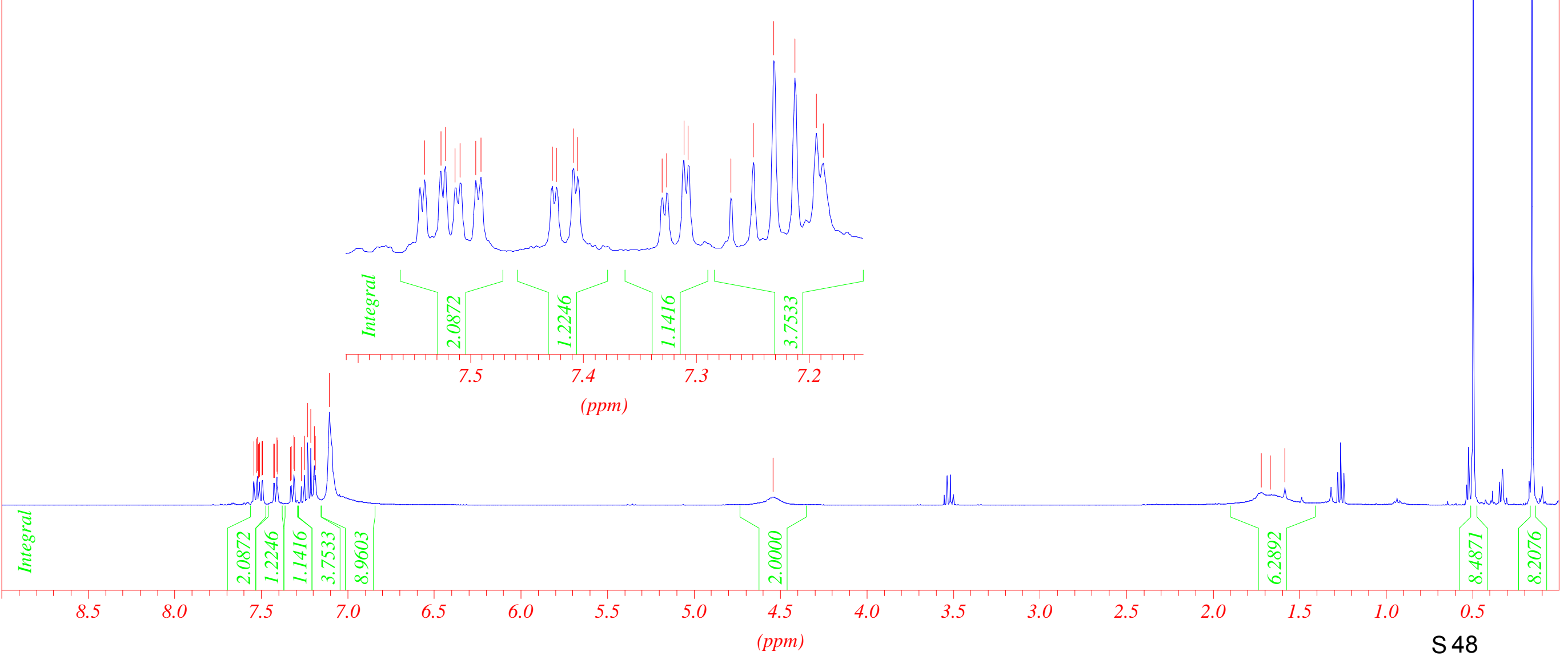



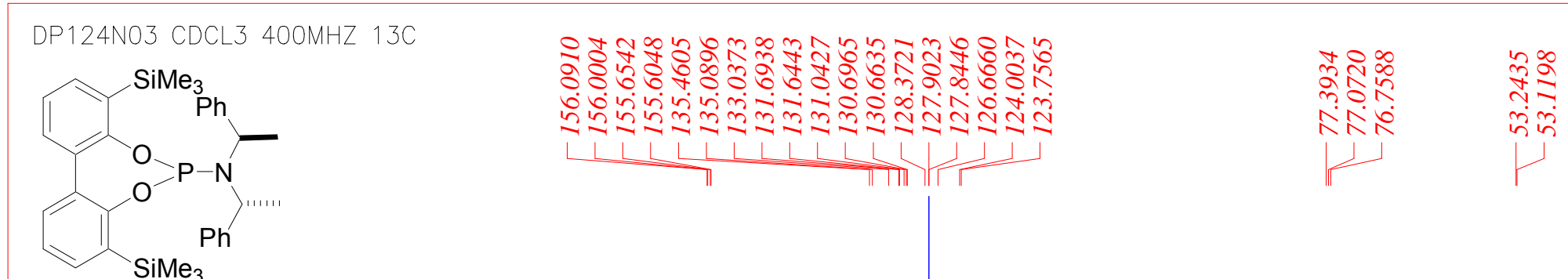

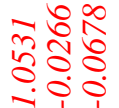

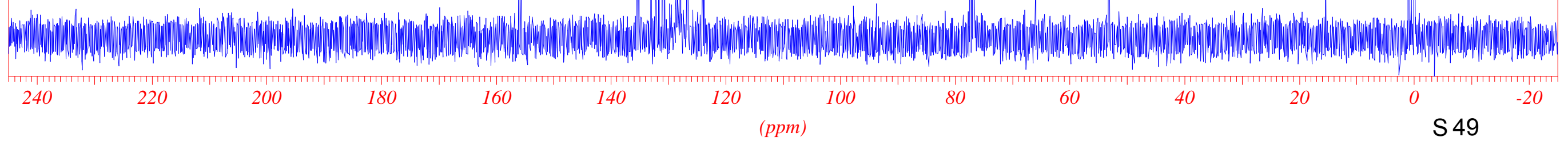


DP124NO3 CDCL3 400MHZ 31P

${ }_{\mathrm{SiMe}_{3}}^{\mathrm{SiMe}_{3}}$

L8a

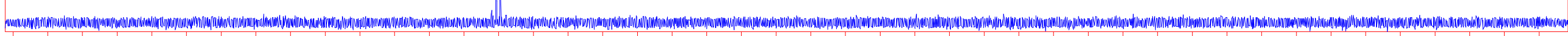


DP077N22 CDCL3 $300 \mathrm{MHZ} 1 \mathrm{H}$

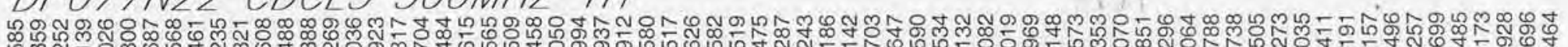

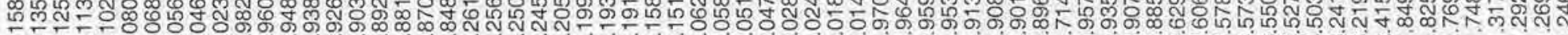

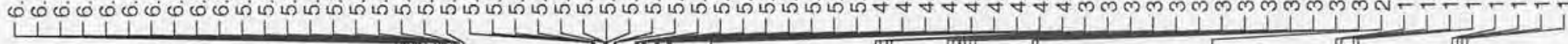

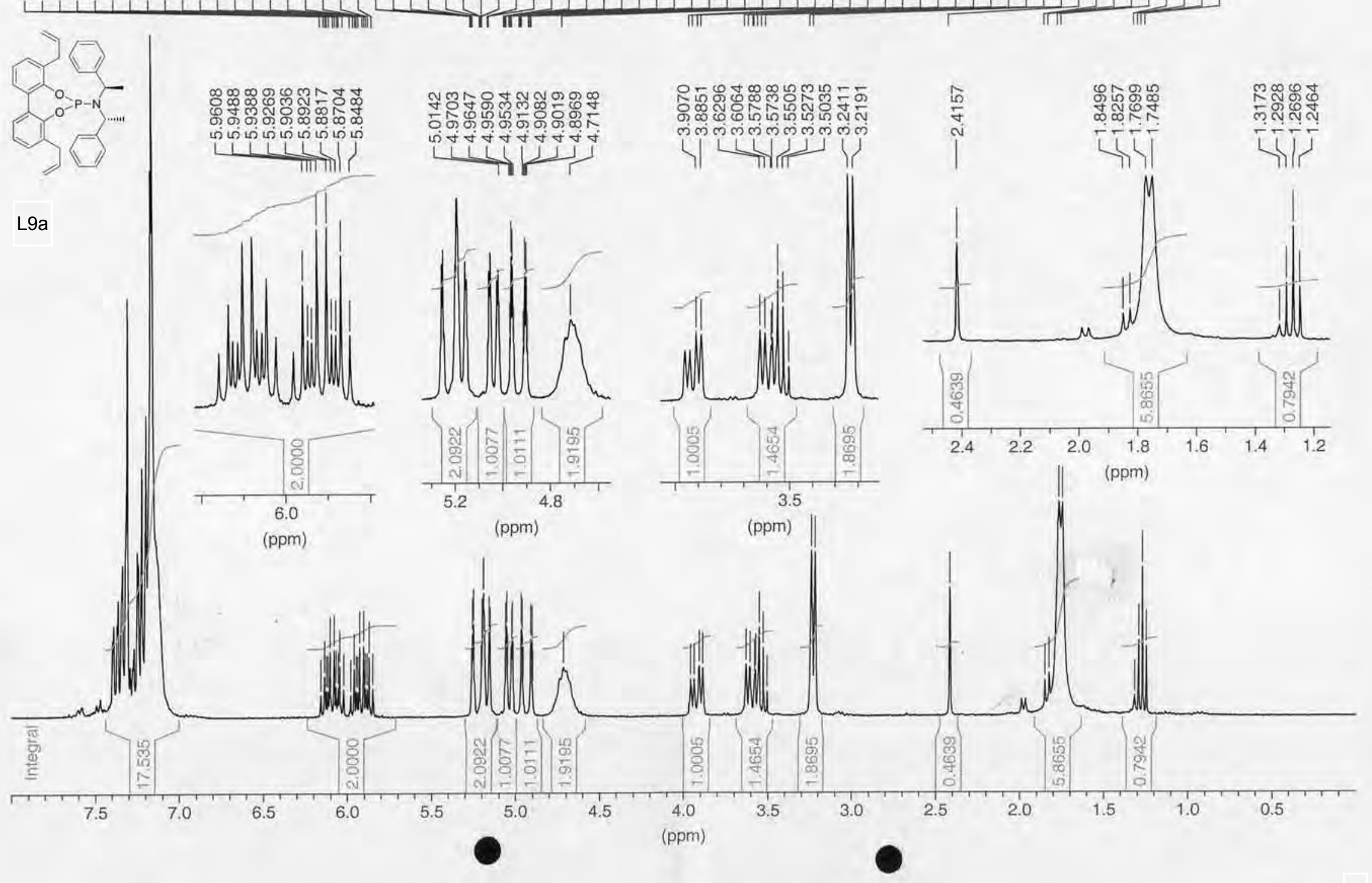




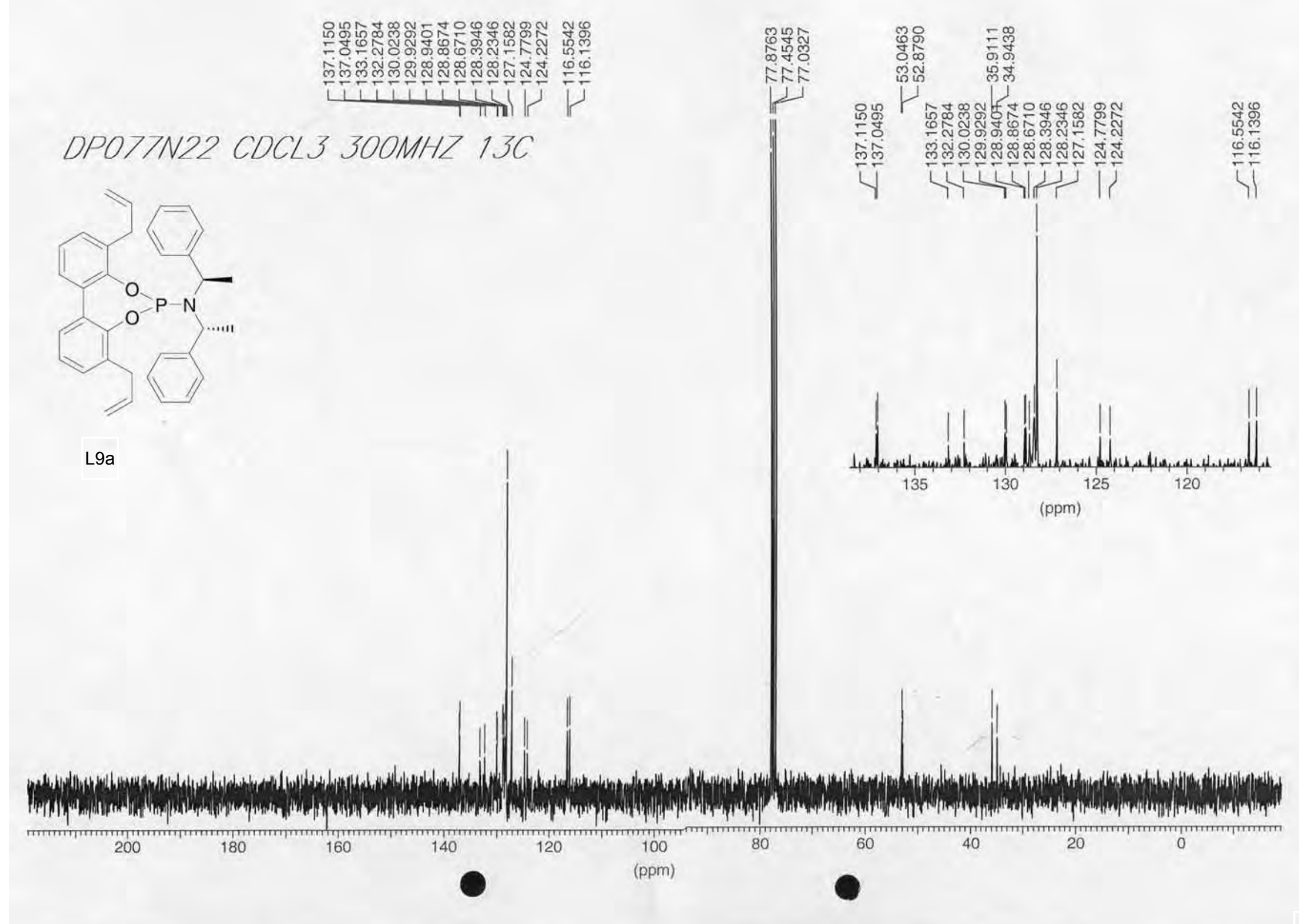




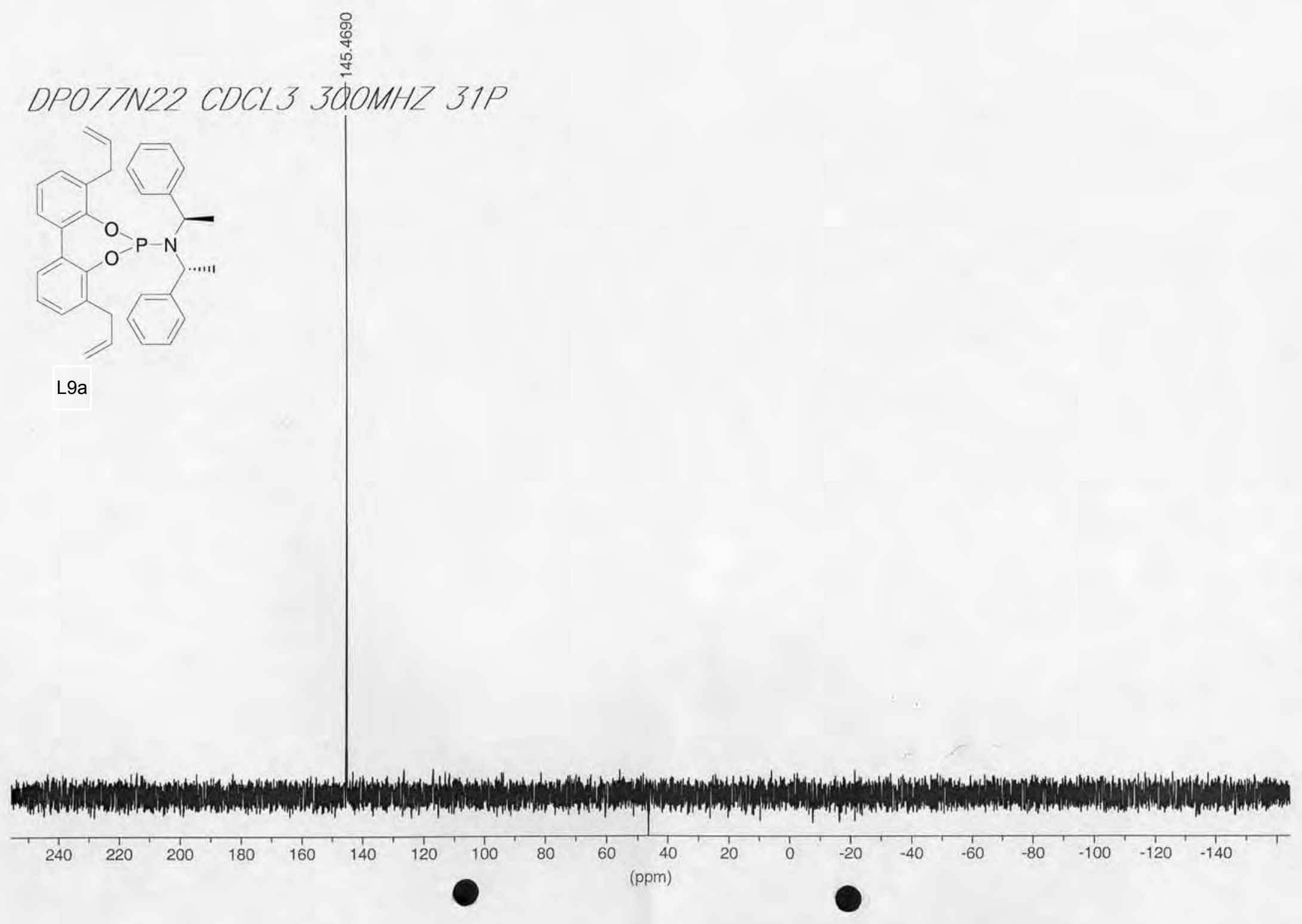




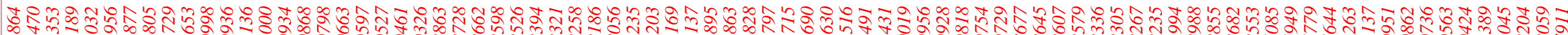

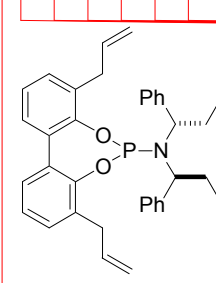

$\mathrm{L9b}$

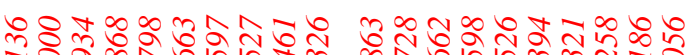

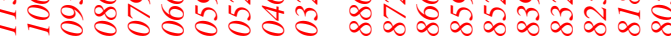

0666666660 hin

IIIII

$7|\pi| \pi \mid$
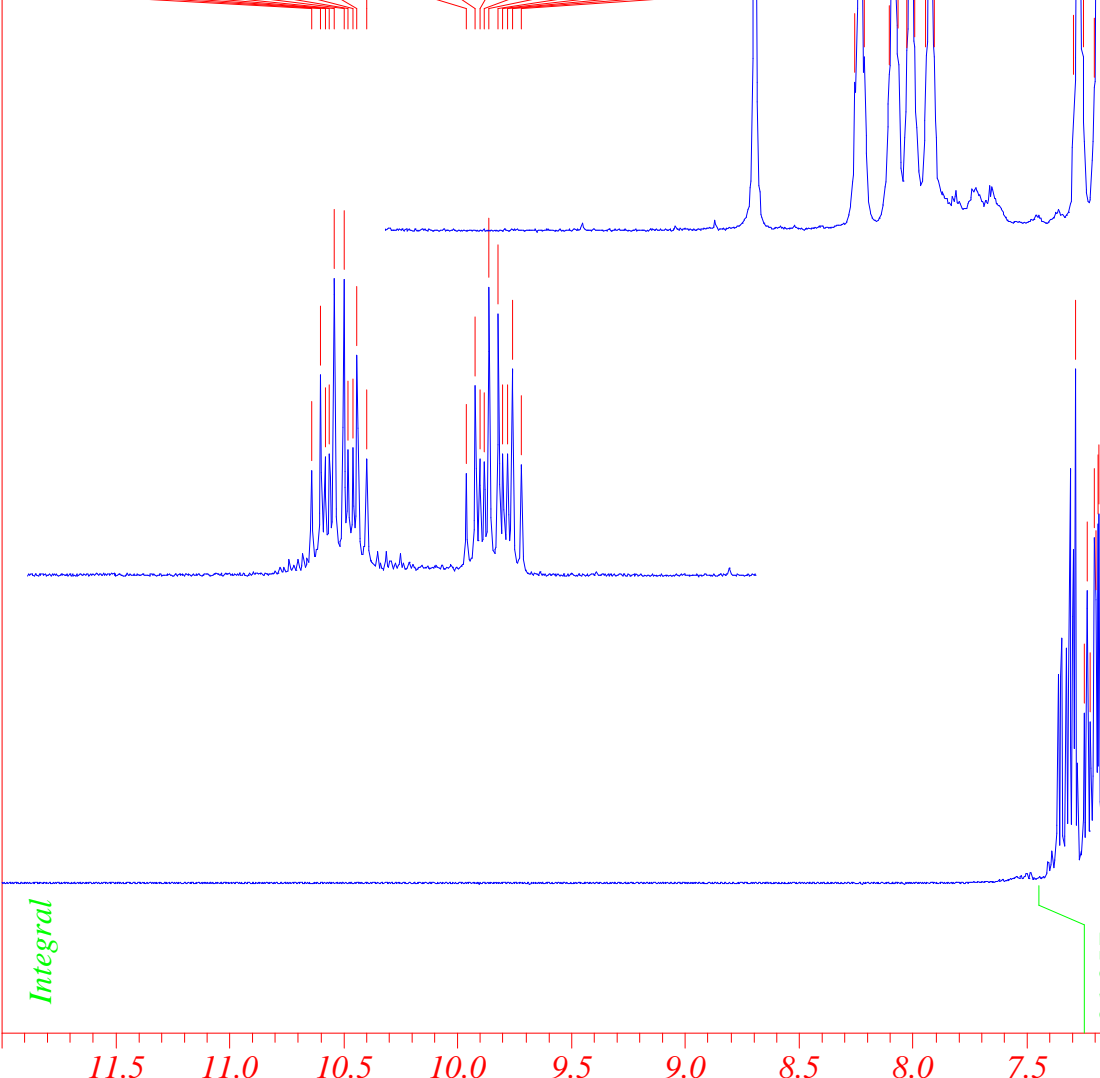

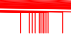

2

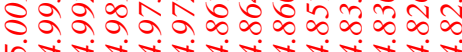

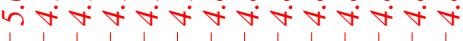

וाIIII
II

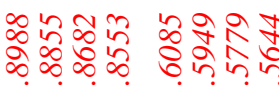
inimi nimminn (l) 


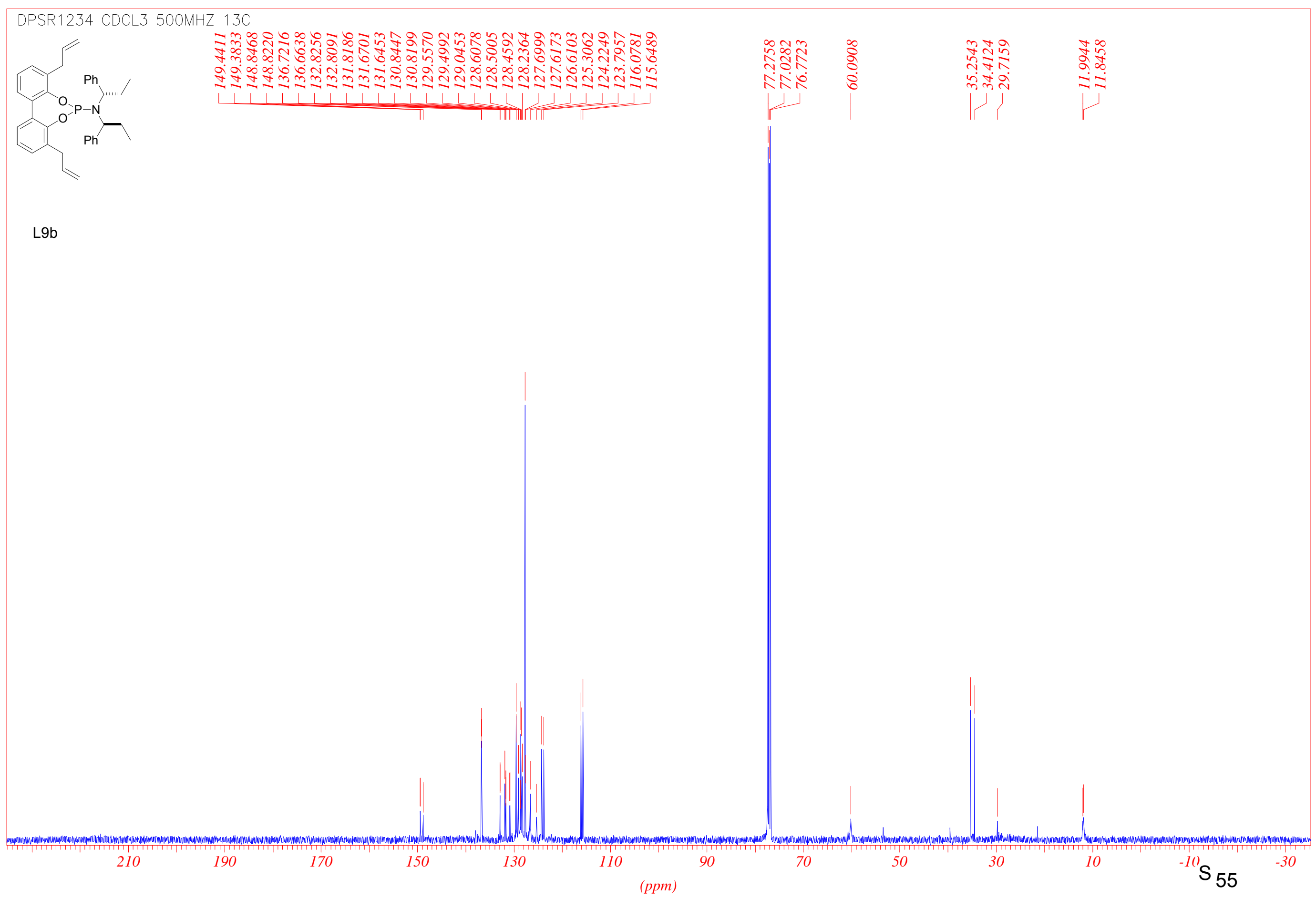




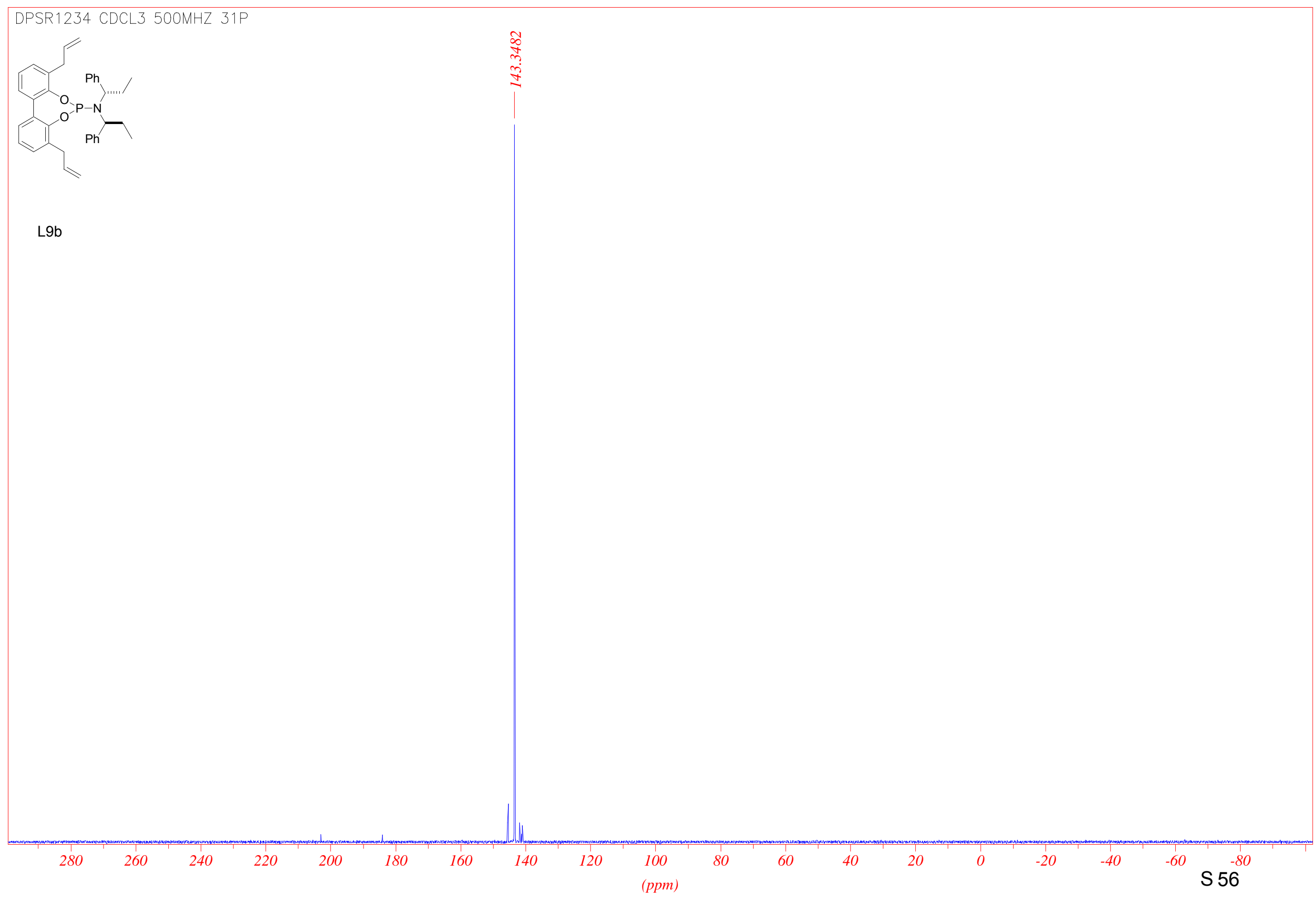




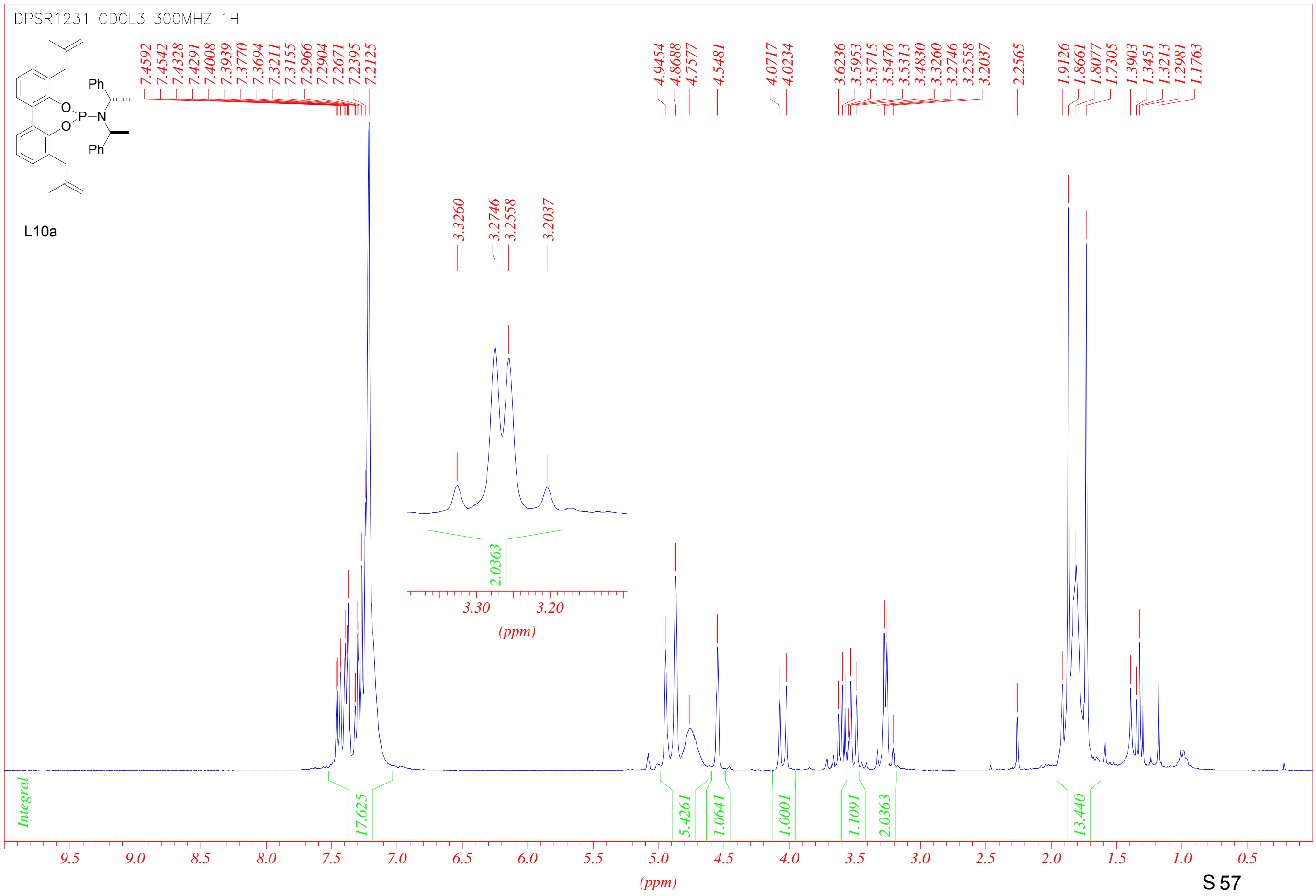




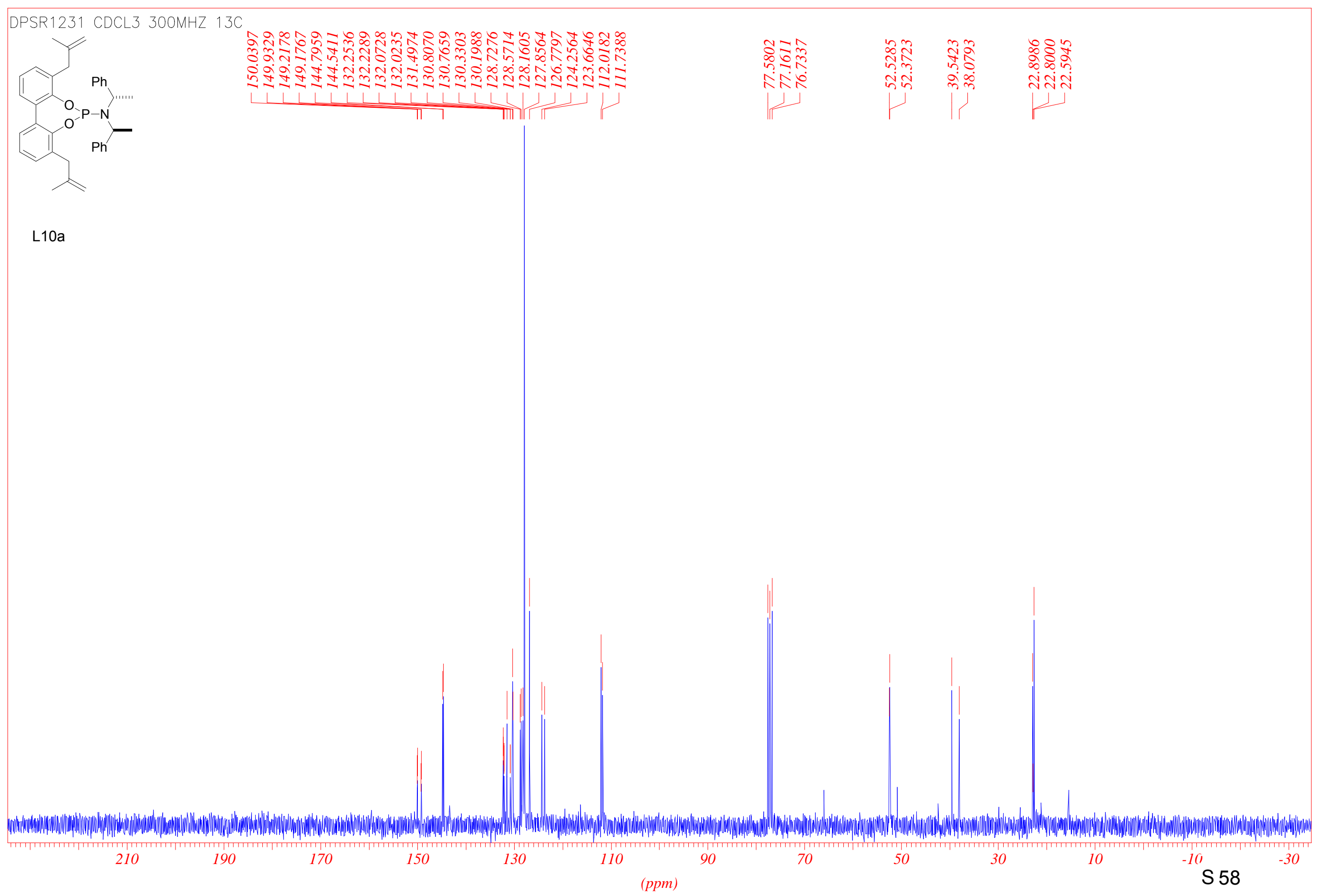




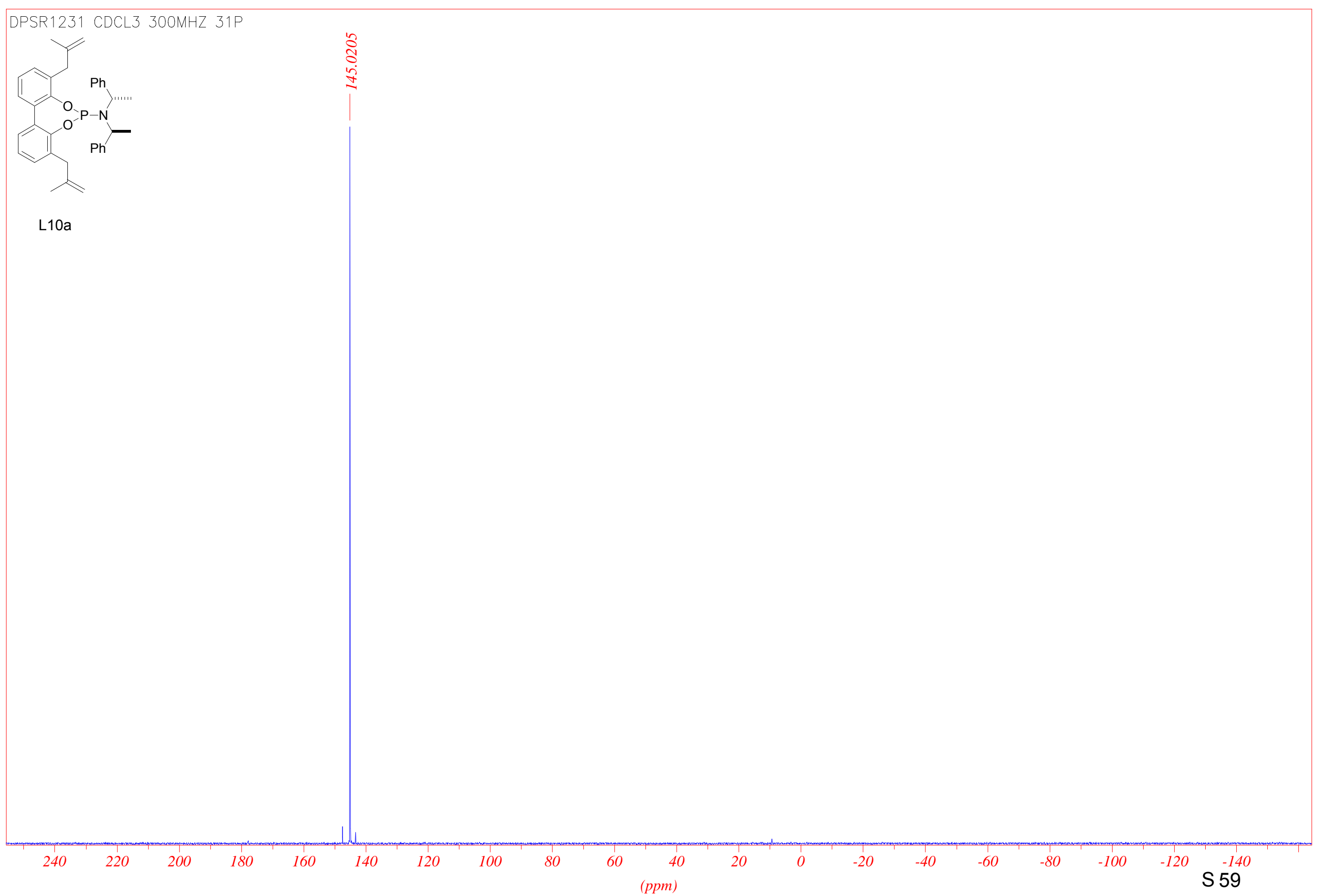




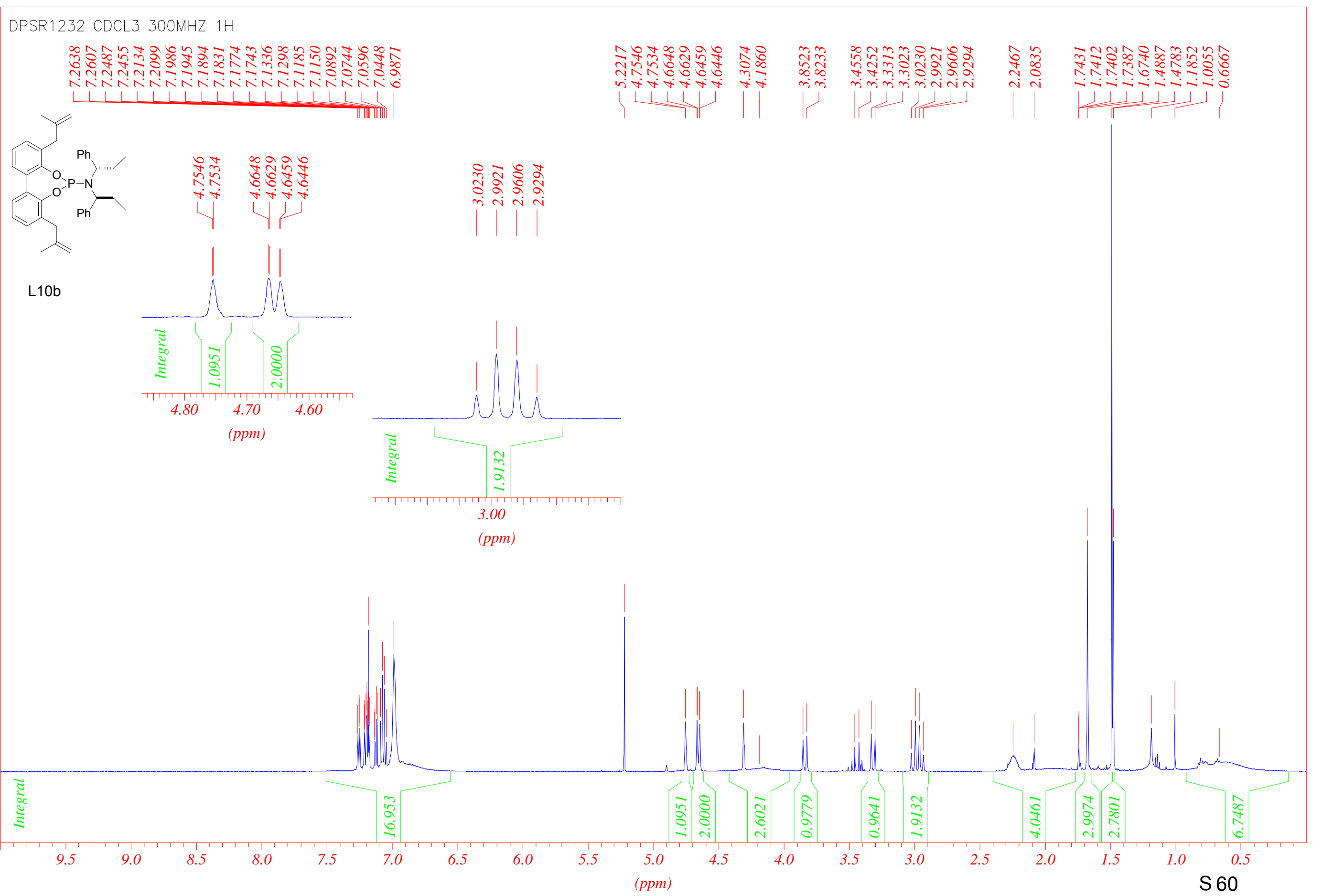




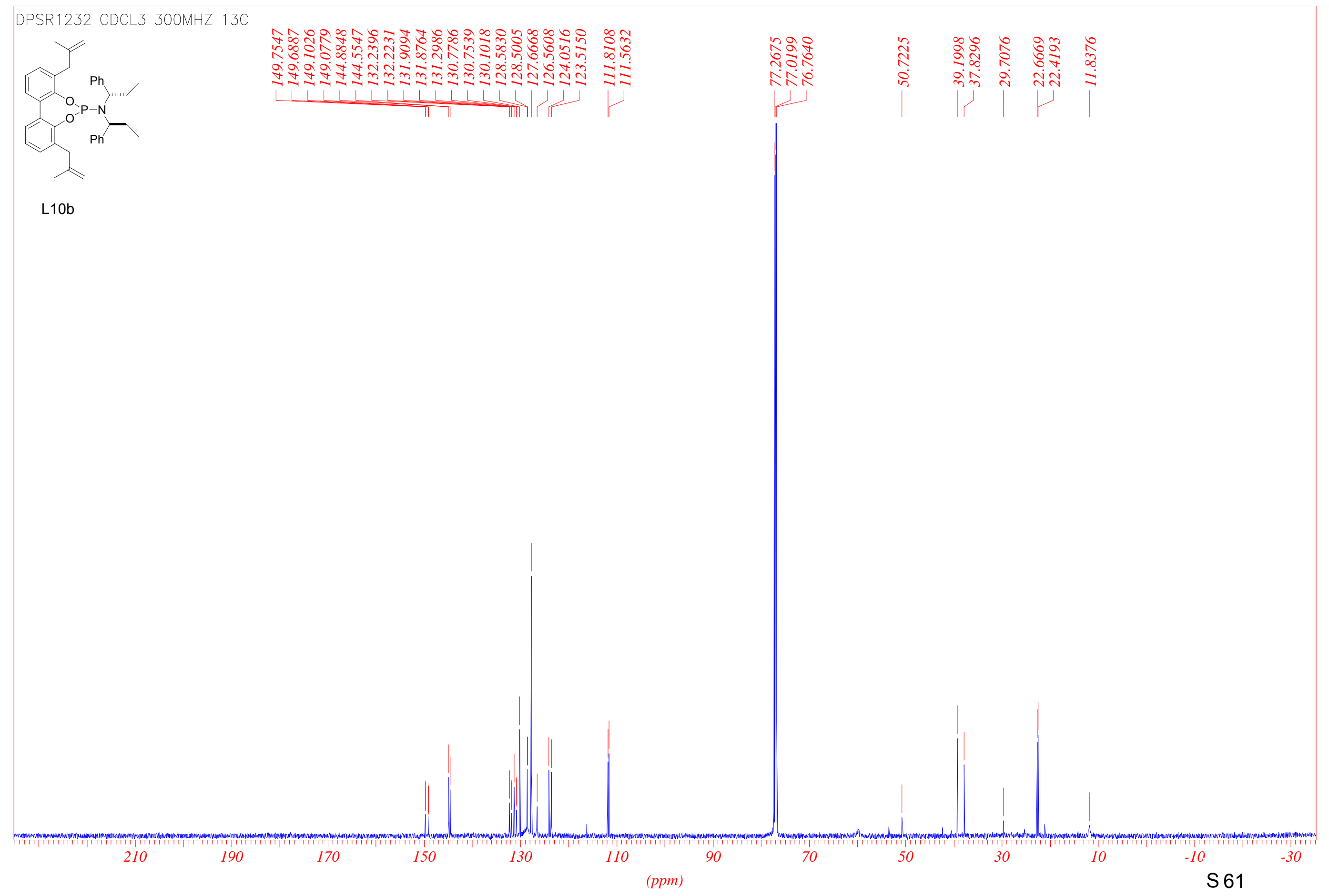




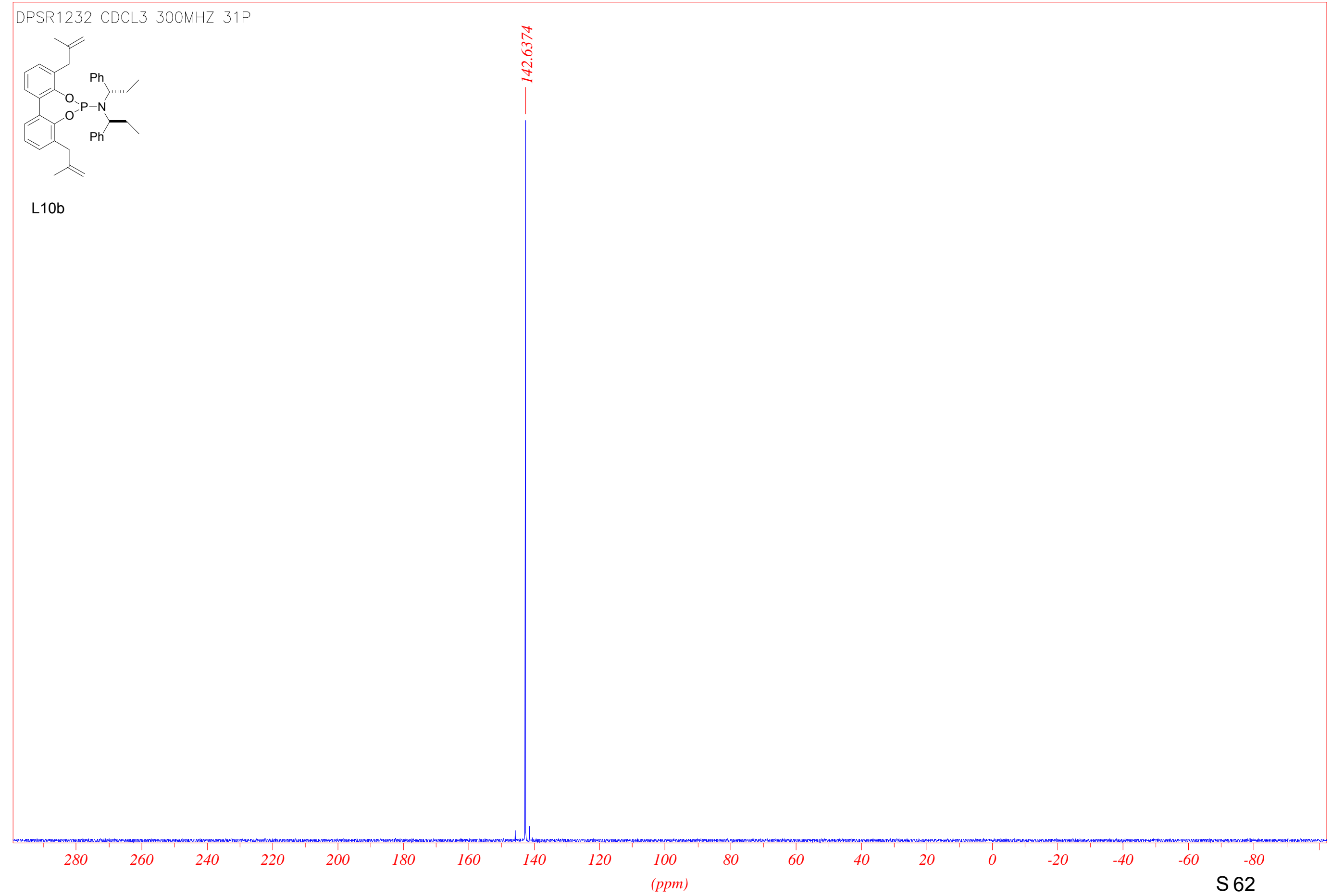


DP194NO6 CDCL3 400MHZ 1H

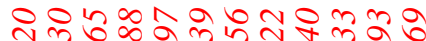

굿ำ

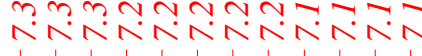

प

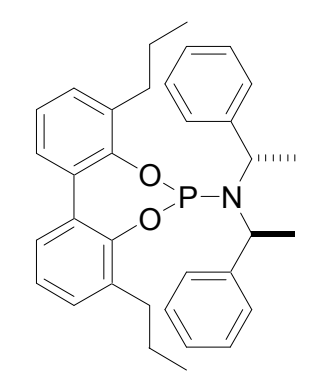

L11a

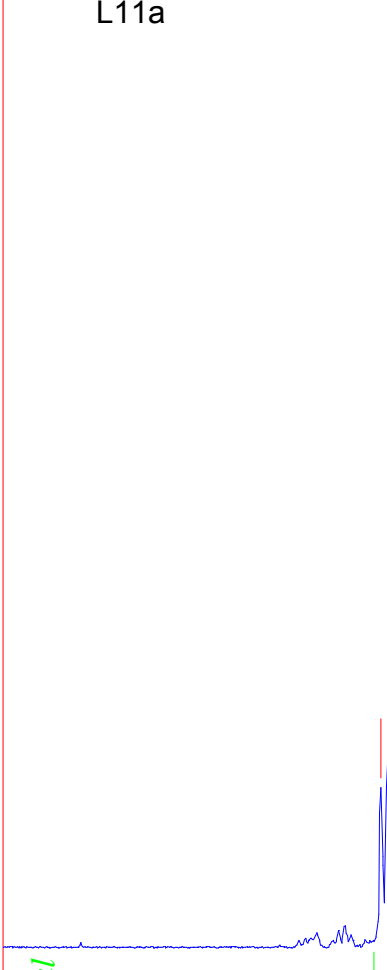

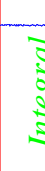

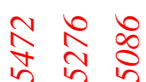

กั

|

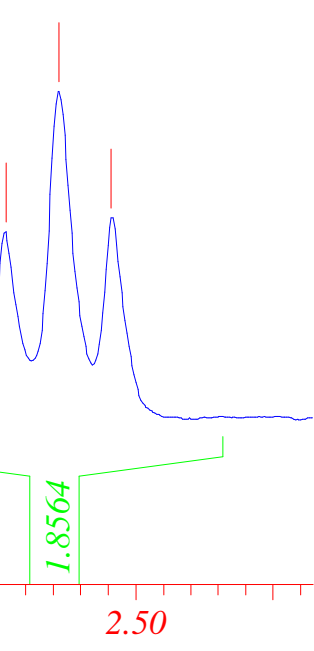

(ppm)
廿

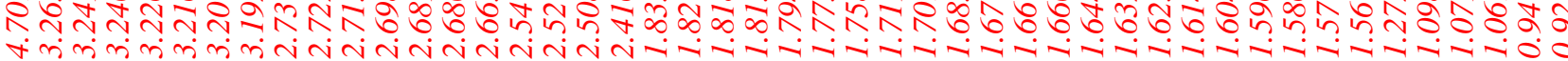

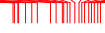

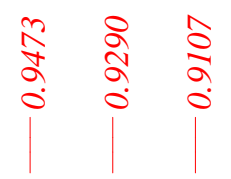

iाIII $\mid \pi$
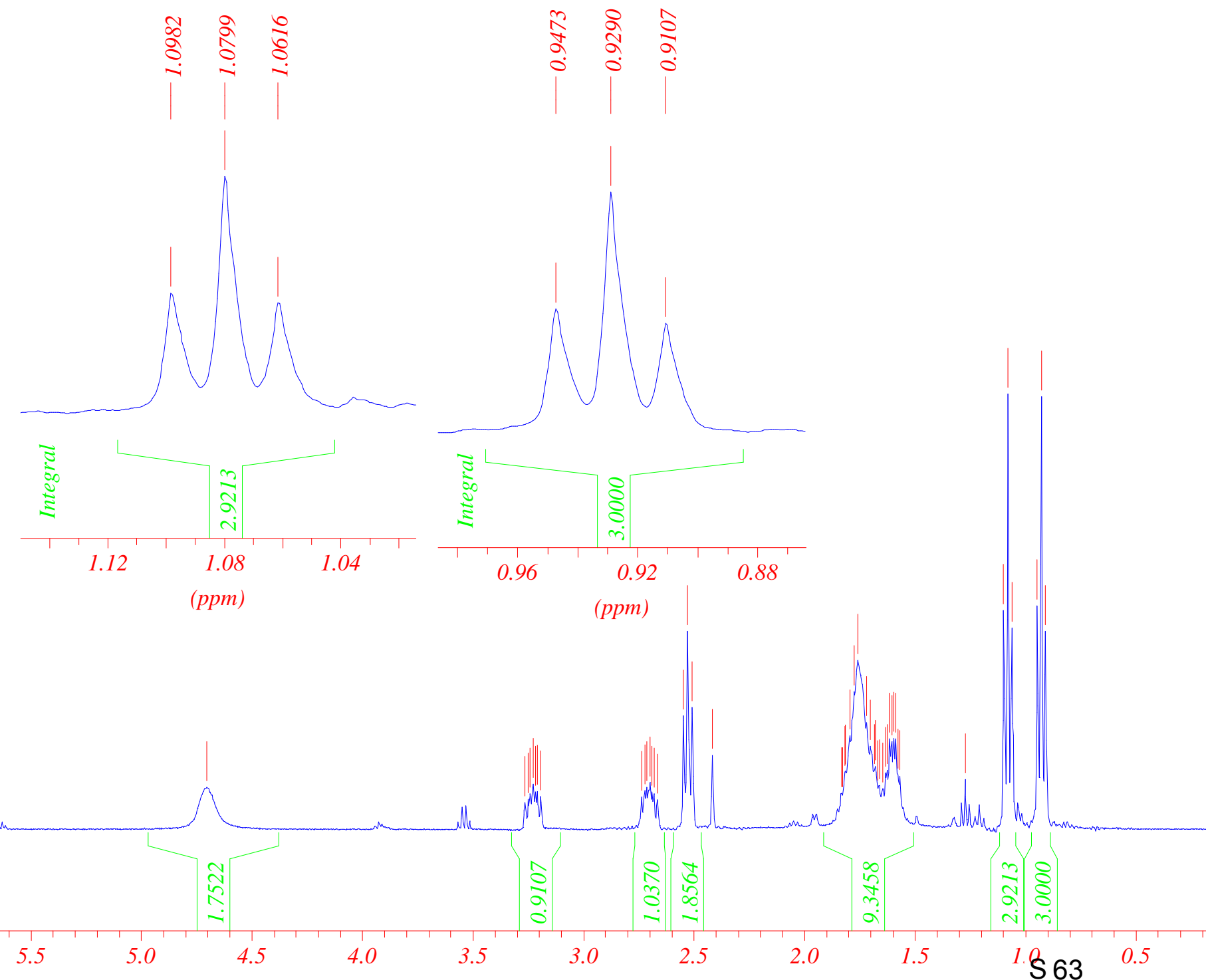


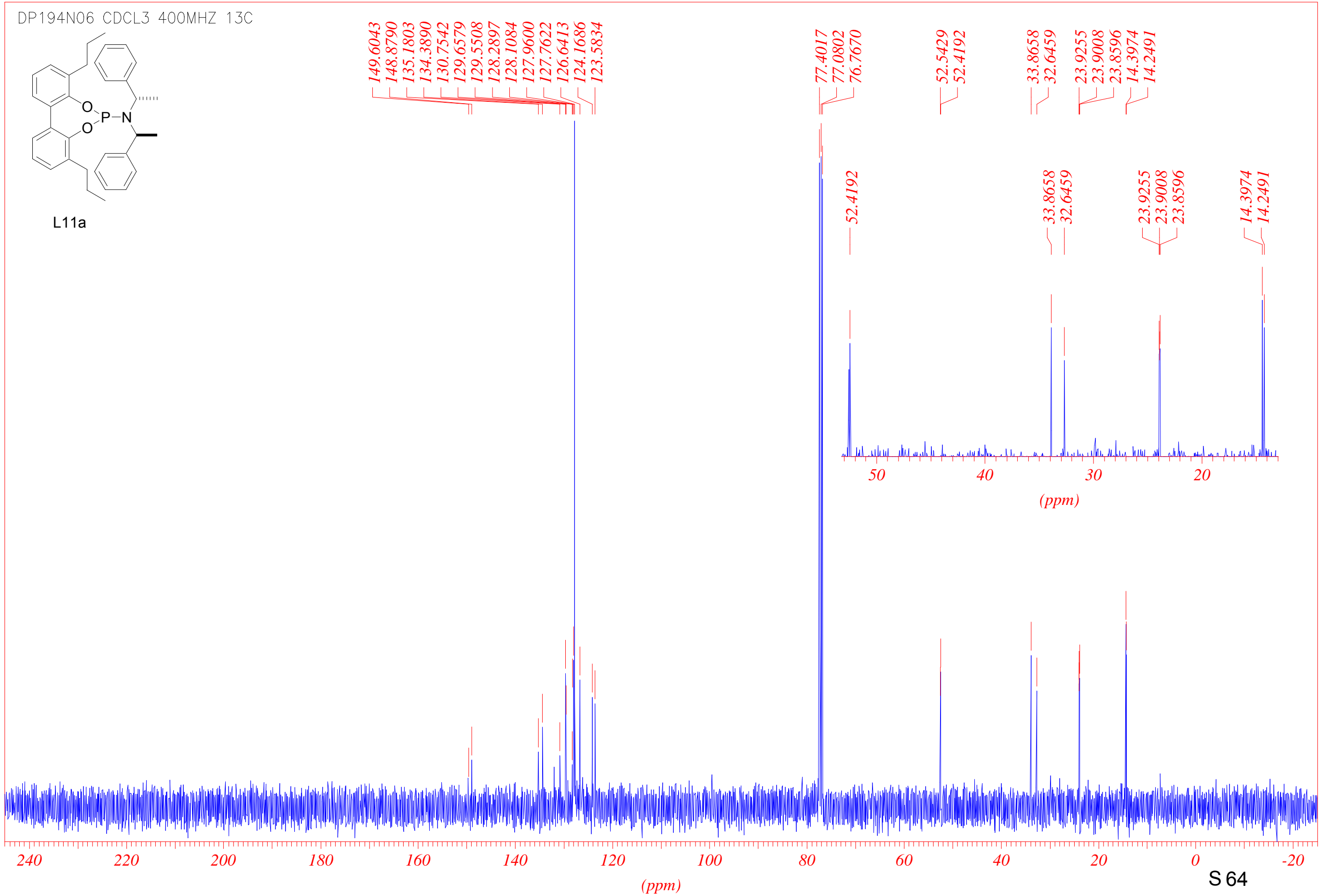


DP194NO6 CDCL3 400MHZ 31P

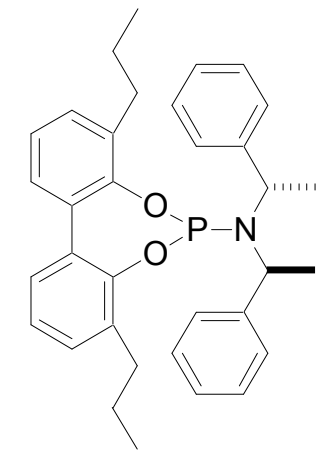

L11a 